\author{
SANDIA REPORT \\ SAND2010-7000 \\ Unlimited Release \\ Printed September 2010
}

\title{
Initiation of the TLR4 Signal Transduction Network - Deeper Understanding For Better Therapeutics
}

Michael S. Kent, Steven Branda, Carl Hayden, Darryl Y. Sasaki, Kenneth L. Sale, Michael Sherman

Prepared by

Sandia National Laboratories

Albuquerque, New Mexico 87185 and Livermore, California 94550

Sandia National Laboratories is a multi-program laboratory managed and operated by Sandia Corporation, a wholly owned subsidiary of Lockheed Martin Corporation, for the U.S. Department of Energy's National Nuclear Security Administration under contract DE-AC04-94AL85000.

Approved for public release; further dissemination unlimited. 
Issued by Sandia National Laboratories, operated for the United States Department of Energy by Sandia Corporation.

NOTICE: This report was prepared as an account of work sponsored by an agency of the United States Government. Neither the United States Government, nor any agency thereof, nor any of their employees, nor any of their contractors, subcontractors, or their employees, make any warranty, express or implied, or assume any legal liability or responsibility for the accuracy, completeness, or usefulness of any information, apparatus, product, or process disclosed, or represent that its use would not infringe privately owned rights. Reference herein to any specific commercial product, process, or service by trade name, trademark, manufacturer, or otherwise, does not necessarily constitute or imply its endorsement, recommendation, or favoring by the United States Government, any agency thereof, or any of their contractors or subcontractors. The views and opinions expressed herein do not necessarily state or reflect those of the United States Government, any agency thereof, or any of their contractors.

Printed in the United States of America. This report has been reproduced directly from the best available copy.

Available to DOE and DOE contractors from

U.S. Department of Energy

Office of Scientific and Technical Information

P.O. Box 62

Oak Ridge, TN 37831

Telephone: $\quad$ (865) 576-8401

Facsimile: (865) 576-5728

E-Mail:_reports@adonis.osti.gov

Online ordering: http://www.osti.gov/bridge

Available to the public from

U.S. Department of Commerce

National Technical Information Service

5285 Port Royal Rd.

Springfield, VA 22161

Telephone: $\quad$ (800) 553-6847

Facsimile: (703) 605-6900

E-Mail: $\quad$ orders@ntis.fedworld.gov

Online order: $\quad$ http://www.ntis.gov/help/ordermethods.asp?loc=7-4-0\#online

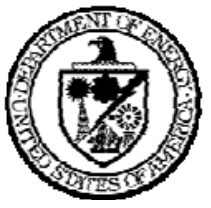


SAND2010-7000

Unlimited Release

Printed September 2010

\title{
Initiation of the TLR4 Signal Transduction Network - Deeper Understanding For Better Therapeutics
}

\author{
Michael S. Kent ${ }^{1}$, Steven Branda ${ }^{2}$, Darryl Y. Sasaki ${ }^{2}$, Carl Hayden ${ }^{3}$, Kenneth L. Sale ${ }^{4}$, Michael \\ Sherman $^{5}$ \\ (1) Bioenergy and Defense Technologies \\ Sandia National Laboratories \\ P.O. Box 5800 \\ Albuquerque, New Mexico 87185-MS1349 \\ (2) Biotechnology \& Bioengineering Department; (3) Combustion Chemistry Department; (4) \\ Biomass Science \& Conversion Technologies Department \\ Sandia National Laboratories \\ P.O. Box 969 \\ Livermore, California 94551-MS9291
}

(5) Dept of Biochemistry and Molecular Biology

1.224 Medical Research Building

University of Texas Medical Branch

301 University Blvd

Galveston, Texas 77555-1055

\begin{abstract}
The innate immune system represents our first line of defense against microbial pathogens, and in many cases is activated by recognition of pathogen cellular components (dsRNA, flagella, LPS, etc.) by cell surface membrane proteins known as toll-like receptors (TLRs). As the initial trigger for innate immune response activation, TLRs also represent a means by which we can effectively control or modulate inflammatory responses. This proposal focused on TLR4, which is the cell-surface receptor primarily responsible for initiating the innate immune response to lipopolysaccharide (LPS), a major component of the outer membrane envelope of gram-negative bacteria. The goal was to better understand TLR4 activation and associated membrane proximal events, in order to enhance the design of small molecule therapeutics to modulate immune activation. Our approach was to reconstitute the receptor in biomimetic systems in-vitro to allow study of the structure and dynamics with biophysical methods. Structural studies were initiated in the first year but were halted after the crystal structure of the dimerized receptor was published
\end{abstract}


early in the second year of the program. Methods were developed to determine the association constant for oligomerization of the soluble receptor. LPS-induced oligomerization was observed to be a strong function of buffer conditions. In $20 \mathrm{mM}$ Tris pH 8.0 with $200 \mathrm{mM} \mathrm{NaCl}$, the onset of receptor oligomerization occurred at $0.2 \mathrm{uM}$ TLR4/MD2 with E coli LPS Ra mutant in excess. However, in the presence of $0.5 \mathrm{uM} \mathrm{CD14}$ and $0.5 \mathrm{uM} \mathrm{LBP}$, the onset of receptor oligomerization was observed to be less than $10 \mathrm{nM}$ TLR4/MD2. Several methods were pursued to study LPS-induced oligomerization of the membrane-bound receptor, including CryoEM, FRET, colocalization and codiffusion followed by TIRF, and fluorescence correlation spectroscopy. However, there approaches met with only limited success. 


\section{ACKNOWLEDGMENTS}

We acknowledge the support of the National Institute of Standards and Technology, U.S. Department of Commerce, in providing the neutron research facilities used in this work. This work also benefited from the use of the Los Alamos Neutron Scattering Center funded by the DOE Office of Basic Energy Sciences and Los Alamos National Laboratory under doe contract DE-AC52-06NA25396. 


\section{CONTENTS}

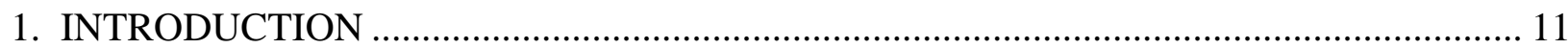

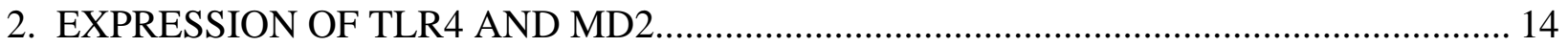

2.1. Commercially available recombinant TLR4 complex units .......................................... 14

2.2. In vitro expression of recombinant proteins ............................................................... 15

2.2.1. In vitro expression of 6xHis-mOrange and 6xHis-mCherry ........................... 15

2.3. E. coli expression of recombinant proteins ................................................................ 25

2.3.1. Expression of 10xHis-hTLR4ed and 6xHis-hMD2........................................ 25

2.4. Summary of recombinant protein expression work ………………………………......... 26

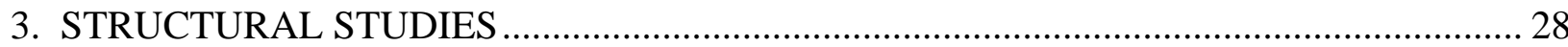

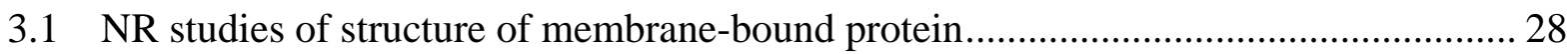

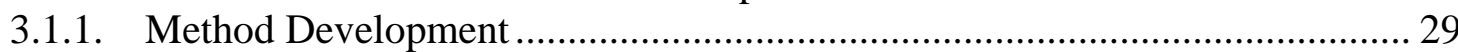

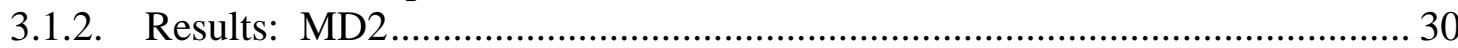

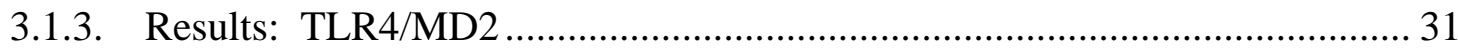

3.1.4. Results: HIV Nef ..................................................................................... 33

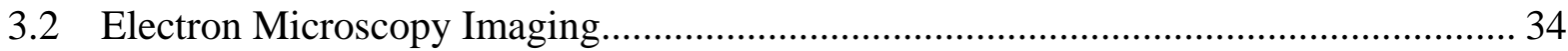

3.2.1. Results: Negative stained EM images of soluble TLR4-MD2 complexes..... 34

3.2.2. CryoEM images of soluble complexes of TLR4-MD2 …………………........ 36

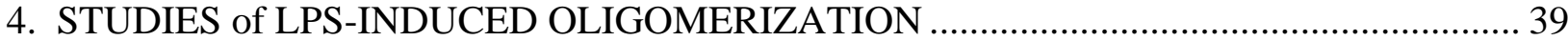

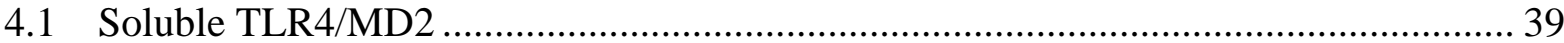

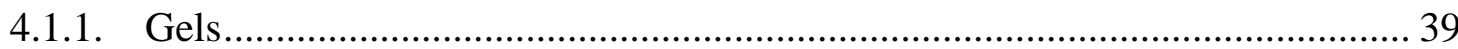

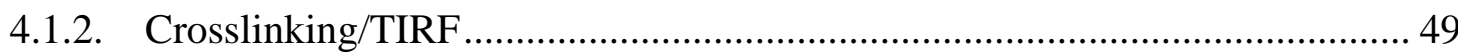

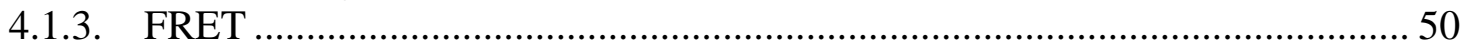

Figure 4-17. Dimension of monomeric and dimeric TLR4/MD2 from recently published crystal structures (Kim et al Nature 2009). The distribution of lysines are shown in the panel on the right. ................................................................................ 51

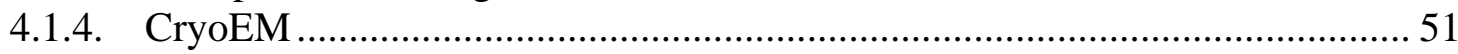

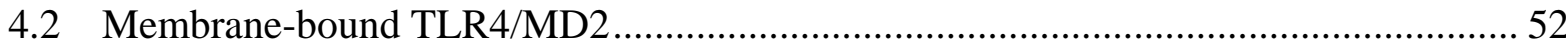

4.2.1. Synthesis of DOIDA ……………………………………………….... 52

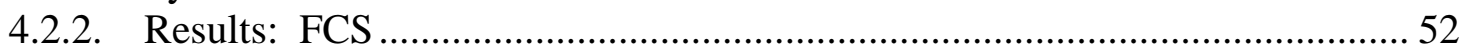

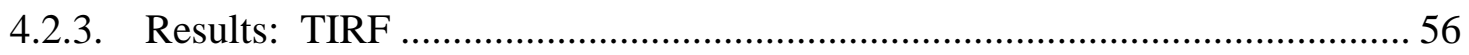

4.2.4. Results: FRET ……………............................................................... 57

4.2.5. Results: CryoEM ...................................................................................... 58

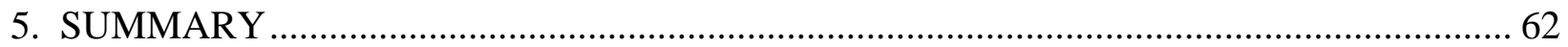

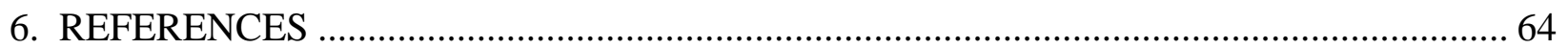

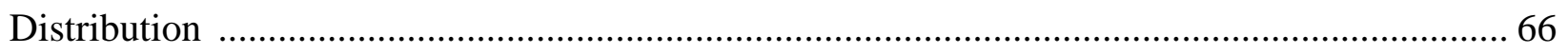

FIGURES

Figure 1-1. Illustration of LPS-induced dimerization of the TLR4 receptor and initiation of intracellular signaling. (taken from Kim et al)........................................................................... 12 


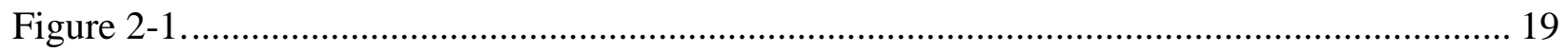

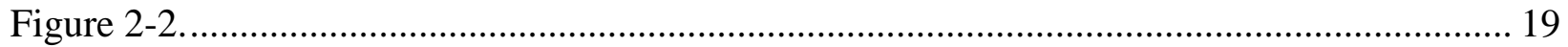

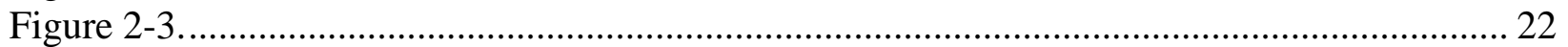

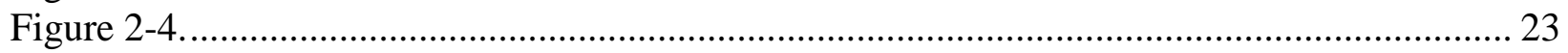

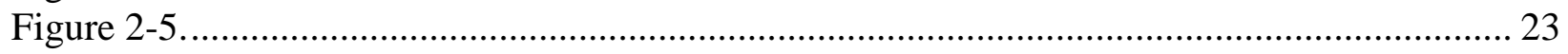

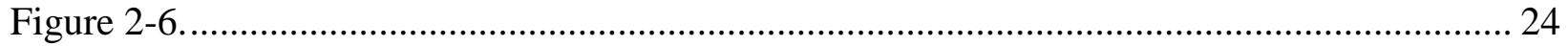

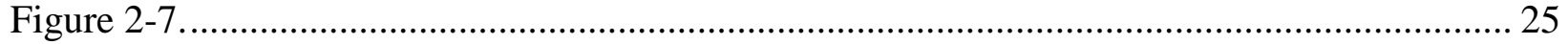

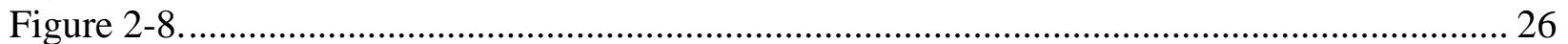

Figure 3-1. NR data for His-tagged MD2 bound to 35\% DSIDA + 65\% d-DPPG and SLD

profiles resulting from the fitting analysis.............................................................................. 31

Figure 3-2. NR data for His-tagged TLR4/MD2 bound to 35\% DSIDA + 65\% d-DPPG. Also shown are calculated SLD profiles and reflectivity for the hypothesized dimeric structure on the

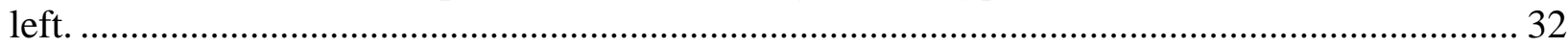

Figure 3-3. NR data for His-tagged d-Nef bound to 35\% d-DSIDA + 65\% d-DPPC................... 33 Figure 3-4. Negatively stained with 2\% uranyl acetate TLR4-MD2 heterodimer. Several particles in different orientations are encircled. 34

Figure 3-5. Upper row: selected characteristic views of TLR4-MD2 heterodimers negatively stained with uranyl acetate. Bottom row: 3D map reprojected in the orientations corresponding the characteristic views. Good matches between the rows showed correct orientation

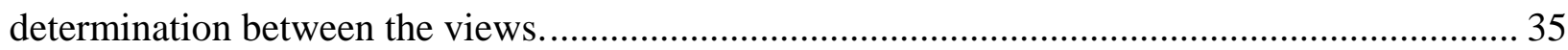

Figure 3-6. TLR4-MD2 dimer (blue-cyan ribbon) fitted into 3D EM map (golden mesh).......... 35 Figure 3-7. TLR4/MD2 resuspended in bufffer and plunge-frozen in liquid ethane. Several individual complexes are encircled.......................................................................................... 37

Figure 4-1. SDS PAGE of TLR4/MD2 samples from R\&D: Lane 1 nonreducing, lane 2

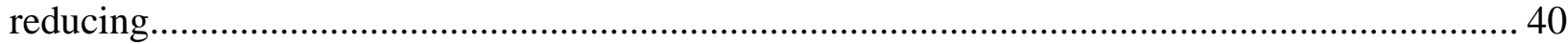

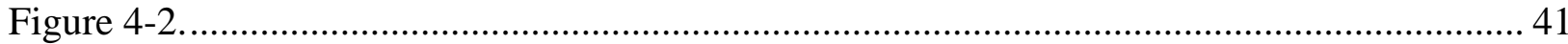

Figure 4-3. Abnova TLR4 combined with R\&D MD2. The band at $55 \mathrm{kDa}$ indicates the presence of dimers of MD2. No TLR4/MD2 complex formation was observed. ........................ 42 Figure 4-4. First observation of LPS-induced oligomerization of TLR4/MD2. Buffer was 20

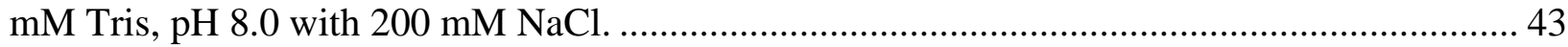

Figure 4-5. SDS PAGE: 2 yr old TLR4 stock, effect of labeling................................................ 44

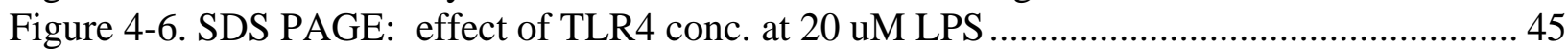

Figure 4-7. SDS PAGE: effect of TLR4 conc. at 20 uM LPS ………….................................. 45

Figure 4-8. SDS PAGE: effect of TLR4 conc. at 20 uM LPS, also control study of glut. conc in absence of LPS. The amount of protein in each lane was constant for the gel on the right side. 46 Figure 4-9. Western blot using primary antibody to MD2 showing LPS-induced oligomerization

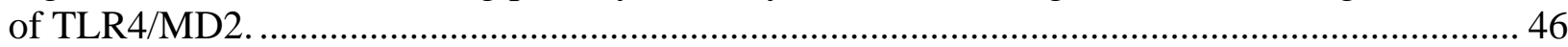

Figure 4-10. Attempt at deglycosylating the TLR4/MD2 sample: SDS PAGE (left), western blot using primary antibody to MD2 (right). ................................................................................ 47

Figure 4-11. Effect of CD14 (left) and LBP (right) on LPS-induced oligomerization of TLR4/MD2. 48

Figure 4-12. Effect of CD14 and LBP. a) Concentration range of TLR4/MD2 extends to down to $0.08 \mathrm{uM}$. b) Concentration range of TLR4/MD2 extends to down to $0.01 \mathrm{uM}$. Strong oligomerization of TLR4 is present at $0.01 \mathrm{uM}$ with LBP and CD14 present. ............................. 48 Figure 4-13. Sample TIRF image and intensity map (1.4 uM TLR4-MD220 uM LPS Ra mut). 49 
Figure 4-14. TIRF intensity distributions for samples corresponding to the gel image on the right

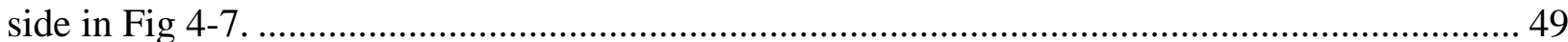
Figure 4-15. Spectra for Alexa 488 (left) and Alexa 555 (right) ................................................ 50 Figure 4-16. Results of FRET study. (left panel) TLR4/MD2 labeled with Alexa 488 and Alexa 555. (center panel) $3.3 \mathrm{uM}$ TLR4/MD2-488 mixed with $3.3 \mathrm{uM}$ TLR4/MD2-555 and incubated with 30 uM LPS Ra mutant. (right panel) ) 1.1 uM TLR4/MD2-488 mixed with $1.1 \mathrm{uM}$ TLR4/MD2-555 but no LPS (control). 50 Figure 4-17. Dimension of monomeric and dimeric TLR4/MD2 from recently published crystal structures (Kim et al Nature 2009). The distribution of lysines are shown in the panel on the right.

Figure 4-18. Fluorescence correlation spectroscopy of a POPC region (blue dots) and a DSIDA region (red dots) of a $10 \%$ DSIDA/POPC membrane following $\mathrm{CuCl}_{2}$ treatment. Fits to the data used to determine diffusion coefficients are shown as black lines. The data were fit to standard correlation function forms for two dimensions. 53 Figure 4-19. Confocal image of a $\mathrm{Cu}^{+2}$-DOIDA/POPC membrane shows binding of proteins in a fluid phase. The protein coverage is quite uniform and the proteins are mobile......................... 53 Figure 4-20. FCS measurement of Atto 532 labeled TLR4/MD2 bound to a DOIDA/POPC membrane. The black fit has a single diffusion coefficient, expected for a fluid membrane...... 54 Figure 4-21. The plot on the left shows the normalized autocorrelation for TLR4/MD2 in solution without LPS added. On the right is a comparison between that autocorrelation and the normalized cross correlation for a sample taken after exposure to LPS. The cross correlation has substantial contribution from aggregates containing both dyes showing it is effective at detecting oligomerization in the sample. 55 Figure 4-22. Normalized cross correlation measurements of a mixture of Alexa Fluor 514 and Atto 590 labeled TLR4/MD2 in the presence and absence of LPS. The cross correlation without LPS results from some crosstalk between the channels and has the same shape as the autocorrelation. The appearance of long time components in the cross correlation with LPS suggests oligomerization..................................................................................................... 55 Figure 4-23. Sample still images of TLR4/MD2-488 and TLR4-MD2-532 bound to DOIDA/POPC membranes.............................................................................................. 56

Figure 4-24. Sample still images of TLR4/MD2-488 and TLR4-MD2-532 bound to DOIDA/POPC membranes. 57

Figure 4-25. Image of negatively stained gold-labeled TLR4/MD2 bound to DOIDA-POPC liposomes. Black dots in the image correspond to gold clusters of $10 \mathrm{~nm}$ in size. In many cases the labels were in close proximity to the liposomes, which confirmed the binding of protein complex to the vesicles. 58 Figure 4-26. CryoEM images of his-tagged TLR4/MD2 bound to liposomes (95\%POPC $+5 \%$ DOIDA) yellow arrows point to side views of dimers of TLR4/MD2 complexes while gold

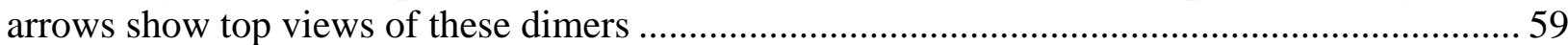
Figure 4-27. CryoEM images of TLR4/MD2 incubated with LPS followed by binding to liposomes. 59

Figure 4-20. Surface rendering of cryoEM reconstruction of dimers of TLR4/MD2 complexes bound to lipid membrane. Top view is shown in A with X-ray structure (Kim, H.M., et al., Cell, 2007. 130, p. 906-17) of TLR4/MD2 fitted into the reconstruction. Dimer of TLR/4MD2 is shown in the same orientation in B without CryoEM map. C and E show side views of cryoEM map with X-ray structure of TLR4/MD2 fitted. D and F show just dimers fitted into cryoEM 
map. Note that dimers are different from published in Park, B.S., et al., Nature, 2009. 458, p.

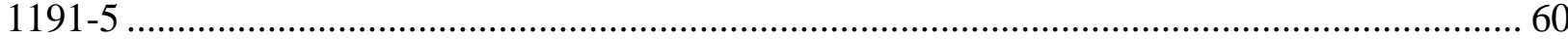




\section{NOMENCLATURE}

DOE Department of Energy

NR Neutron Reflectivity

Tris

PBS 


\section{INTRODUCTION}

The innate immune system represents our first line of defense against microbial pathogens, and in many cases is activated by recognition of pathogen cellular components (dsRNA, flagella, LPS, etc.) by cell surface membrane proteins known as toll-like receptors (TLRs). As the initial trigger for innate immune response activation, TLRs also represent a means by which we can effectively control or modulate inflammatory responses. However, much remains to be discovered about the molecular mechanisms underlying TLR activation. This work focused on TLR4 (see Fig 1), which is the cell-surface receptor primarily responsible for initiating the innate immune response to lipopolysaccharide (LPS), a major component of the bacterial cell envelope. Our overarching goal was to better understand TLR4 activation and associated membrane proximal events, which will greatly enhance the design of small molecule therapeutics to modulate immune activation. Prior studies have shown a strong LPS specificity for human TLR4/MD2 (e.g. lipid A and lipid IVa, Cell 2007, 130, 906), and also important variations in signaling response to LPS chemotypes among TLR4/MD2 receptor complexes from different mammalian species (J. of Immunol. 2008, 181, 1245). During this project, the crystal structure of dimeric human TLR4/MD2 (ectodomain) with bound ligand was published (Nature pub. online Mar 1, 2009). This showed that TLR4/MD2 dimers are stabilized by rather subtle interactions upon LPS binding; a hydrophobic interaction between an exposed tail of MD2bound LPS and conserved phenylalanines of TLR4, and ionic interactions between the phosphate groups of LPS and positively charged residues of TLR4 and MD2. These results suggest that variations in the number of acyl chains of LPS or in specific residues of TLR4 and MD2 at the dimer interface will alter the stability of the heterotetrameric complex. Thus, small changes in the dimer-monomer equilibrium or in the average lifetime of a dimer may explain the wide range of signaling responses observed. We developed a model system and biophysical probes to examine the equilibrium and dynamics of ligand-induced dimerization of TLR4/MD2 with the goal of correlating these data with variations in signaling in live cells. This approach provides a platform and methods for testing molecular mechanisms, including the actions of adjuvants and therapeutics, directly at the receptor level. This should facilitate the rational design of inflammation modulators.

An effort was made throughout the course of this program to express the soluble portion of the receptor recombinantly. The primary motivation for this was to enable study of the effect of specific mutations on receptor association, and also to enable the placement of fluorescent labels in precise locations for energy transfer studies. This effort is described in section 2. Section 3 summarizes the structural studies aimed at resolving the structure of the dimerized receptor complex. Studies of LPS-induced oligomerization of the TLR4 receptor are reported in section 4 . Section 5 contains a summary of the most important conclusions from this work. 


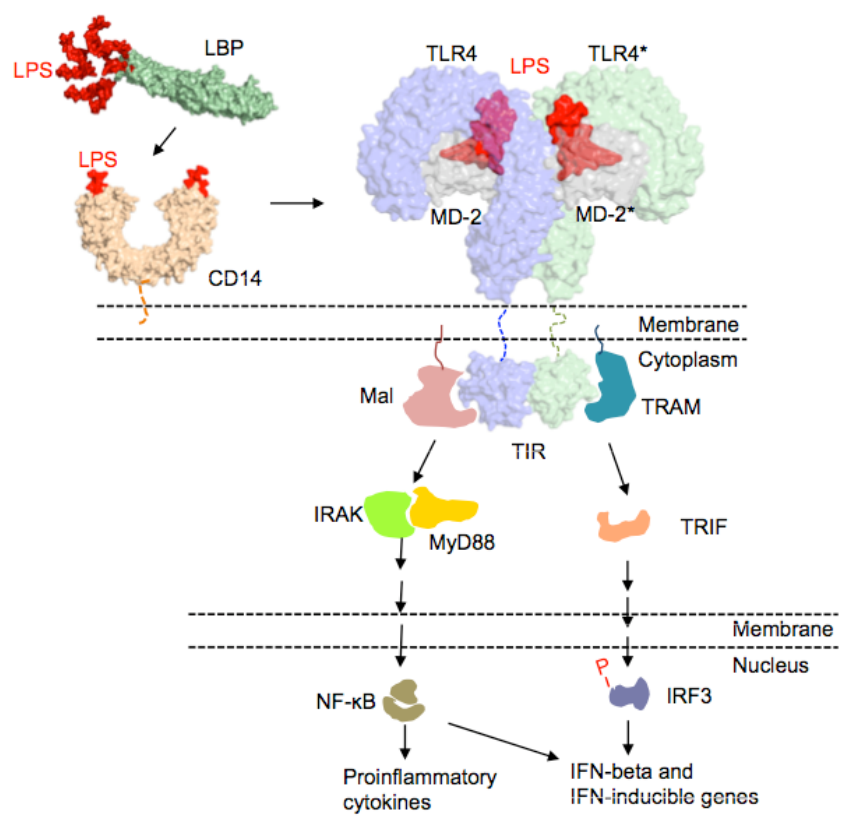

Figure 1-1. Illustration of LPS-induced dimerization of the TLR4 receptor and initiation of intracellular signaling. (taken from Kim et al) 


\section{EXPRESSION OF TLR4 AND MD2}

\subsection{Commercially available recombinant TLR4 complex units}

To study TLR4 receptor complex composition and dynamics we sought sources of recombinant forms of the receptor complex subunits: TLR4, MD2, CD14, and LBP. In the cases of TLR4 and MD2, which are membrane-bound proteins in the cell, we sought recombinant versions bearing His tags, to facilitate our work with $\mathrm{Cu}$-impregnated lipid bilayers.

R\&D Systems sells a recombinant human TLR4/MD2 complex (cat\# 3146-TM-050). Each of the subunits is the mature form (TLR4: Glu24 - Lys631; MD2: Glu17 - Asn160) and bear a 10xHis tag at the C-terminus. The proteins are expressed in a murine myeloma cell line; presumably they are expressed simultaneously, rather than separately and then mixed to form the complex, though R\&D Systems would not comment on that.

R\&D Systems also sells recombinant mature (Glu17 - Asn160) human MD2 with a C-terminal 10xHis tag (cat\# 1787-MD), similar to the MD2 subunit in the TLR4/MD2 complex described above. However, unlike the complex's subunit, the lone MD2 is expressed by Escherichia coli. MD2 (and TLR4) is $N$-glycosylated in mammalian cells, and E. coli is incapable of $N$ glycosylating recombinant proteins. So the complex's MD2 (and TLR4) subunit should be glycosylated, whereas the lone MD2 is not. There is conflicting evidence as to whether glycosylation of TLR4 and/or MD2 impacts LPS binding and/or TLR4-mediated signaling; our philosophy is to work with glycosylated forms of the proteins whenever possible.

R\&D Systems also sells recombinant mature (Thr20 - Cys352) human CD14 expressed by the Chinese Hamster Ovary (CHO) cell line (cat\# 383-CD-050), as well as recombinant mature (Ala26 - Val481) human LBP expressed by a murine myeloma cell line (presumably the same one used for expression of the TLR4/MD2 complex) (cat\# 870-LP). Both subunits should be glycosylated.

Much of our work took advantage of the availability of these commercially-available recombinant proteins. However, there were several drawbacks to using them. Without direct control over their expression and purification we were wholly dependent upon R\&D Systems to produce "good" proteins (i.e., stable, pure, functional), and could not produce variants of the proteins (e.g., separately expressed TLR4, mutant versions of the proteins) for our own purposes. Moreover, the commercial preps were expensive (e.g., the TLR4/MD2 complex was $\$ 315$ for 30 $\mu \mathrm{g})$.

Therefore, there was strong incentive to produce recombinant proteins of our own. We evaluated the expression systems available, and eliminated two on the basis of difficulty in start-up (baculovirus + insect cells, and yeast) and a third on the basis of extremely low yield (mammalian cells). Of the remaining systems, we prioritized the cell-free approach over E. coli expression because it is simpler, faster, and capable of producing adequate quantities of recombinant proteins for our purposes. Moreover, a potential drawback to E. coli expression is that the bacteria's LPS could come through in the protein prep and remain bound to the recombinant proteins, altering their ability to interact with one another. The most robust of the 
cell-free systems depend upon E. coli lysates, which may be contaminated with LPS; however, if their yields are acceptable the many non-E. coli based cell-free systems would circumvent this potential problem altogether. As a compromise we initiated our expression studies using E. coli cell-free systems, with the idea that if expression were very robust we could switch to another, non-E. coli based cell-free system; and if expression were weak we could switch to using intact E. coli.

\subsection{In vitro expression of recombinant proteins}

\subsubsection{In vitro expression of $6 x$ His-mOrange and 6xHis-mCherry}

There are many different non-E. coli cell-free systems to choose from, and we needed a good positive control recombinant protein to rationally choose between them. Moreover, our lipid bilayer and microscopy studies would benefit from our ability to recombinantly express Histagged fluorescent proteins. Therefore, we designed and generated PCR products that could be used to support cell-free expression of recombinant mOrange and mCherry fluorescent proteins that were 6xHis tagged at either the $\mathrm{N}$ - or C-terminus. We based the PCR constructs on guidance from the Qiagen EasyXpress Linear Template Kit Plus (cat\# 32723), which not only supports cell-free expression but also cloning of the PCR products into the pIX 3.0 vector (cat\# 32733) for expression in E. coli.

The mOrange and mCherry ORFs are 97.5\% identical at the nucleotide level, so it was possible to use the same primers to $6 x$ His tag both proteins. The primer sequences are as follows:

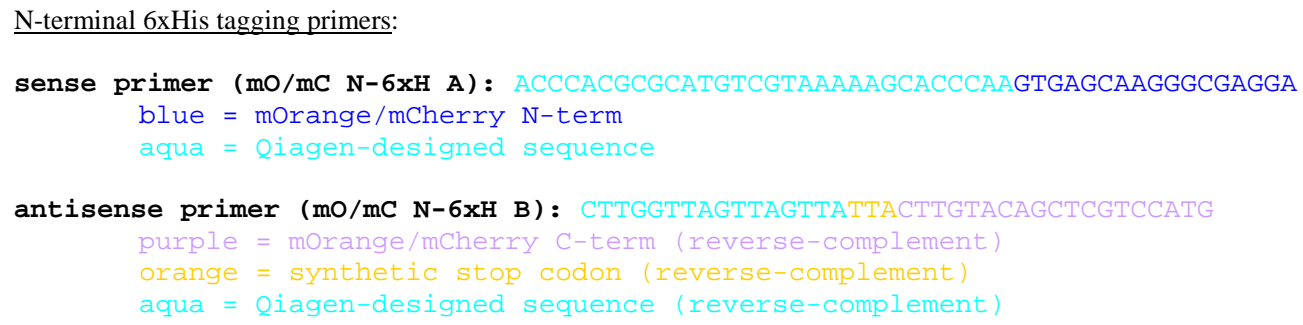

The plasmids pBSK-mOrange and pBSK-mCherry (received from Cathy Branda) were used as templates in the PCR reactions, and ran a portion of the completed reaction on an agarose gel to confirm that sufficient product was generated. The products supported robust expression of N- 
terminal 6xHis-tagged fluorescent proteins, as measured through fluorimetry; the C-terminal 6xHis-tagged proteins showed weaker expression.

To generate a more stable source of each construct, for larger-scale expression of the recombinant proteins, we cloned the N-terminal 6xHis-tagged mOrange and mCherry PCR products into the pIX 3.0 vector. The multi-stage PCR, cloning, and diagnostic restriction digest strategies are as follows:

\section{Product of 1st round of PCR for $\mathrm{N}$-term His-tagged morange}

ACCCACGCGCATGTCGTAAAAAGCACCCAAGTGAGCAAGGGCGAGGAGAATAACATGGCCATCATCAAGGAGTTCATGCGCTTCAAGGTGCGCATGG AGGGCTCCGTGAACGGCCACGAGTTCGAGATCGAGGGCGAGGGCGAGGGCCGCCCCTACGAGGGCTTTCAGACCGCTAAGCTGAAGGTGACCAAGGG TGGCCCCCTGCCCTTCGCCTGGGACATCCTGTCCCCTCAGTTCACCTACGGCTCCAAGGCCTACGTGAAGCACCCCGCCGACATCCCCGACTACTTC AAGCTGTCCTTCCCCGAGGGCTTCAAGTGGGAGCGCGTGATGAACTTCGAGGACGGCGGCGTGGTGACCGTGACCCAGGACTCCTCCCTGCAGGACG GCGAGTTCATCTACAAGGTGAAGCTGCGCGGCACCAACTTCCCCTCCGACGGCCCCGTAATGCAGAAGAAGACCATGGGCTGGGAGGCCTCCTCCGA GCGGATGTACCCCGAGGACGGCGCCCTGAAGGGCGAGATCAAGATGAGGCTGAAGCTGAAGGACGGCGGCCACTACACCTCCGAGGTCAAGACCACC TACAAGGCCAAGAAGCCCGTGCAGCTGCCCGGCGCCTACATCGTCGGCATCAAGTTGGACATCACCTCCCACAACGAGGACTACACCATCGTGGAAC AGTACGAACGCGCCGAGGGCCGCCACTCCACCGGCGGCATGGACGAGCTGTACAAGTAATAACTAACTAACCAAG

\section{Product of 2nd (\& 3rd) round of PCR for $\mathrm{N}$-term His-tagged morange} ATGATATCTCGAGCGGCCGCTAGCTAATACGACTCACTATAGGGAGACCACAACGGTTTCCCTCTAGAAATAATTTTGTTTAACTTTAAGAAGGAGA TAAACAATGAAACATCATCACCATCACCACTCGACCCACGCGCATGTCGTAAAAAGCACCCAAGTGAGCAAGGGCGAGGAGAATAACATGGCCATCA TCAAGGAGTTCATGCGCTTCAAGGTGCGCATGGAGGGCTCCGTGAACGGCCACGAGTTCGAGATCGAGGGCGAGGGCGAGGGCCGCCCCTACGAGGG CTTTCAGACCGCTAAGCTGAAGGTGACCAAGGGTGGCCCCCTGCCCTTCGCCTGGGACATCCTGTCCCCTCAGTTCACCTACGGCTCCAAGGCCTAC GTGAAGCACCCCGCCGACATCCCCGACTACTTCAAGCTGTCCTTCCCCGAGGGCTTCAAGTGGGAGCGCGTGATGAACTTCGAGGACGGCGGCGTGG TGACCGTGACCCAGGACTCCTCCCTGCAGGACGGCGAGTTCATCTACAAGGTGAAGCTGCGCGGCACCAACTTCCCCTCCGACGGCCCCGTAATGCA GAAGAAGACCATGGGCTGGGAGGCCTCCTCCGAGCGGATGTACCCCGAGGACGGCGCCCTGAAGGGCGAGATCAAGATGAGGCTGAAGCTGAAGGAC GGCGGCCACTACACCTCCGAGGTCAAGACCACCTACAAGGCCAAGAAGCCCGTGCAGCTGCCCGGCGCCTACATCGTCGGCATCAAGTTGGACATCA CCTCCCACAACGAGGACTACACCATCGTGGAACAGTACGAACGCGCCGAGGGCCGCCACTCCACCGGCGGCATGGACGAGCTGTACAAGTAATAACT AACTAACCAAGATCTGTACCCCTTGGGGCCTCTAAACGGGTCTTGAGGGGTTTTTTGGATCCGAATTCACCGGTGATATCAT

\begin{tabular}{|c|c|c|}
\hline yellow & EcoRI & $\mathrm{G}^{\wedge} \mathrm{AATTC}$ \\
\hline green & BamHI & $\mathrm{G}^{\wedge} \mathrm{GATCC}$ \\
\hline & EcoRV & GAT^ATC \\
\hline purple & NheI & $\mathrm{G}^{\wedge} \mathrm{CTAGC}$ \\
\hline grey & XhoI & $\mathrm{C}^{\wedge} \mathrm{TCGAG}$ \\
\hline turquoise & PVuII & $\mathrm{CAG}^{\wedge} \mathrm{CTG}$ \\
\hline & EagI & $\mathrm{C}^{\wedge} \mathrm{GGCCG}$ \\
\hline & PstT & CTGCA \\
\hline
\end{tabular}

\section{NheI + EcoRI digest of $\mathrm{N}$-term His-tagged morange}

CTAGCTAATACGACTCACTATAGGGAGACCACAACGGTTTCCCTCTAGAAATAATTTTGTTTAACTTTAAGAAGGAGATAAACAATGAAACATCATC ACCATCACCACTCGACCCACGCGCATGTCGTAAAAAGCACCCAAGTGAGCAAGGGCGAGGAGAATAACATGGCCATCATCAAGGAGTTCATGCGCTT CAAGGTGCGCATGGAGGGCTCCGTGAACGGCCACGAGTTCGAGATCGAGGGCGAGGGCGAGGGCCGCCCCTACGAGGGCTTTCAGACCGCTAAGCTG AAGGTGACCAAGGGTGGCCCCCTGCCCTTCGCCTGGGACATCCTGTCCCCTCAGTTCACCTACGGCTCCAAGGCCTACGTGAAGCACCCCGCCGACA TCCCCGACTACTTCAAGCTGTCCTTCCCCGAGGGCTTCAAGTGGGAGCGCGTGATGAACTTCGAGGACGGCGGCGTGGTGACCGTGACCCAGGACTC CTCCCTGCAGGACGGCGAGTTCATCTACAAGGTGAAGCTGCGCGGCACCAACTTCCCCTCCGACGGCCCCGTAATGCAGAAGAAGACCATGGGCTGG GAGGCCTCCTCCGAGCGGATGTACCCCGAGGACGGCGCCCTGAAGGGCGAGATCAAGATGAGGCTGAAGCTGAAGGACGGCGGCCACTACACCTCCG AGGTCAAGACCACCTACAAGGCCAAGAAGCCCGTGCAGCTGCCCGGCGCCTACATCGTCGGCATCAAGTTGGACATCACCTCCCACAACGAGGACTA CACCATCGTGGAACAGTACGAACGCGCCGAGGGCCGCCACTCCACCGGCGGCATGGACGAGCTGTACAAGTAATAACTAACTAACCAAGATCTGTAC CCCTTGGGGCCTCTAAACGGGTCTTGAGGGGTTTTTTGGATCCG

NheI + EcoRI frag of $\mathrm{N}$-term His-tagged morange cloned in pIX3.0

TCGCGCGTTTCGGTGATGACGGTGAAAACCTCTGACACATGCAGCTCCCGGAGACGGTCACAGCTTGTCTGTAAGCGGATGCCGGGAGCAGACAAGC CCGTCAGGGCGCGTCAGCGGGTGTTGGCGGGTGTCGGGGCTGGCTTAACTATGCGGCATCAGAGCAGATTGTACTGAGAGTGCACCATATGCGGTGT GAAATACCGCACAGATGCGTAAGGAGAAAATACCGCATCAGGCGCCATTCGCCATTCAGGCTGCGCAACTGTTGGGAAGGGCGATCGGTGCGGGCCT CTTCGCTATTACGCCAGCTGGCGAAAGGGGGATGTGCTGCAAGGCGATTAAGTTGGGTAACGCCAGGGTTTTCCCAGTCACGACGTTGTAAAACGAC GGCCAGTGCCAAGCTTTCTCGAGCGGCCGCTAGCTAATACGACTCACTATAGGGAGACCACAACGGTTTCCCTCTAGAAATAATTTTGTTTAACTTT AAGAAGGAGATAAACAATGAAACATCATCACCATCACCACTCGACCCACGCGCATGTCGTAAAAAGCACCCAAGTGAGCAAGGGCGAGGAGAATAAC ATGGCCATCATCAAGGAGTTCATGCGCTTCAAGGTGCGCATGGAGGGCTCCGTGAACGGCCACGAGTTCGAGATCGAGGGCGAGGGCGAGGGCCGCC CCTACGAGGGCTTTCAGACCGCTAAGCTGAAGGTGACCAAGGGTGGCCCCCTGCCCTTCGCCTGGGACATCCTGTCCCCTCAGTTCACCTACGGCTC CAAGGCCTACGTGAAGCACCCCGCCGACATCCCCGACTACTTCAAGCTGTCCTTCCCCGAGGGCTTCAAGTGGGAGCGCGTGATGAACTTCGAGGAC GGCGGCGTGGTGACCGTGACCCAGGACTCCTCCCTGCAGGACGGCGAGTTCATCTACAAGGTGAAGCTGCGCGGCACCAACTTCCCCTCCGACGGCC CCGTAATGCAGAAGAAGACCATGGGCTGGGAGGCCTCCTCCGAGCGGATGTACCCCGAGGACGGCGCCCTGAAGGGCGAGATCAAGATGAGGCTGAA GCTGAAGGACGGCGGCCACTACACCTCCGAGGTCAAGACCACCTACAAGGCCAAGAAGCCCGTGCAGCTGCCCGGCGCCTACATCGTCGGCATCAAG 
TTGGACATCACCTCCCACAACGAGGACTACACCATCGTGGAACAGTACGAACGCGCCGAGGGCCGCCACTCCACCGGCGGCATGGACGAGCTGTACA AGTAATAACTAACTAACCAAGATCTGTACCCCTTGGGGCCTCTAAACGGGTCTTGAGGGGTTTTTTTGGATCCGAATTCACCGGTGCAATTCGTAATC ATGTCATAGCTGTTTCCTGTGTGAAATTGTTATCCGCTCACAATTCCACACAACATACGAGCCGGAAGCATAAAGTGTAAAGCCTGGGGTGCCTAAT GAGTGAGCTAACTCACATTAATTGCGTTGCGCTCACTGCCCGCTTTCCAGTCGGGAAACCTGTCGTGCCAGCTGCATTAATGAATCGGCCAACGCGC GGGGAGAGGCGGTTTGCGTATTGGGCGCTCTTCCGCTTCCTCGCTCACTGACTCGCTGCGCTCGGTCGTTCGGCTGCGGCGAGCGGTATCAGCTCAC TCAAAGGCGGTAATACGGTTATCCACAGAATCAGGGGATAACGCAGGAAAGAACATGTGAGCAAAAGGCCAGCAAAAGGCCAGGAACCGTAAAAAGG CCGCGTTGCTGGCGTTTTTCCATAGGCTCCGCCCCCCTGACGAGCATCACAAAAATCGACGCTCAAGTCAGAGGTGGCGAAACCCGACAGGACTATA AAGATACCAGGCGTTTCCCCCTGGAAGCTCCCTCGTGCGCTCTCCTGTTCCGACCCTGCCGCTTACCGGATACCTGTCCGCCTTTCTCCCTTCGGGA AGCGTGGCGCTTTCTCATAGCTCACGCTGTAGGTATCTCAGTTCGGTGTAGGTCGTTCGCTCCAAGCTGGGCTGTGTGCACGAACCCCCCGTTCAGC CCGACCGCTGCGCCTTATCCGGTAACTATCGTCTTGAGTCCAACCCGGTAAGACACGACTTATCGCCACTGGCAGCAGCCACTGGTAACAGGATTAG CAGAGCGAGGTATGTAGGCGGTGCTACAGAGTTCTTGAAGTGGTGGCCTAACTACGGCTACACTAGAAGAACAGTATTTGGTATCTGCGCTCTGCTG AAGCCAGTTACCTTCGGAAAAAGAGTTGGTAGCTCTTGATCCGGCAAACAAACCACCGCTGGTAGCGGTGGTTTTTTTGTTTGCAAGCAGCAGATTA CGCGCAGAAAAAAAGGATCTCAAGAAGATCCTTTGATCTTTTCTACGGGGTCTGACGCTCAGTGGAACGAAAACTCACGTTAAGGGATTTTGGTCAT GAGATTATCAAAAAGGATCTTCACCTAGATCCTTTTAAATTAAAAATGAAGTTTTAAATCAATCTAAAGTATATATGAGTAAACTTGGTCTGACAGT TACCAATGCTTAATCAGTGAGGCACCTATCTCAGCGATCTGTCTATTTCGTTCATCCATAGTTGCCTGACTCCCCGTCGTGTAGATAACTACGATAC GGGAGGGCTTACCATCTGGCCCCAGTGCTGCAATGATACCGCGAGACCCACGCTCACCGGCTCCAGATTTATCAGCAATAAACCAGCCAGCCGGAAG GGCCGAGCGCAGAAGTGGTCCTGCAACTTTATCCGCCTCCATCCAGTCTATTAATTGTTGCCGGGAAGCTAGAGTAAGTAGTTCGCCAGTTAATAGT TTGCGCAACGTTGTTGCCATTGCTACAGGCATCGTGGTGTCACGCTCGTCGTTTGGTATGGCTTCATTCAGCTCCGGTTCCCAACGATCAAGGCGAG TTACATGATCCCCCATGTTGTGCAAAAAAGCGGTTAGCTCCTTCGGTCCTCCGATCGTTGTCAGAAGTAAGTTGGCCGCAGTGTTATCACTCATGGT TATGGCAGCACTGCATAATTCTCTTACTGTCATGCCATCCGTAAGATGCTTTTCTGTGACTGGTGAGTACTCAACCAAGTCATTCTGAGAATAGTGT ATGCGGCGACCGAGTTGCTCTTGCCCGGCGTCAATACGGGATAATACCGCGCCACATAGCAGAACTTTAAAAGTGCTCATCATTGGAAAACGTTCTT CGGGGCGAAAACTCTCAAGGATCTTACCGCTGTTGAGATCCAGTTCGATGTAACCCACTCGTGCACCCAACTGATCTTCAGCATCTTTTACTTTCAC CAGCGTTTCTGGGTGAGCAAAAACAGGAAGGCAAAATGCCGCAAAAAAGGGAATAAGGGCGACACGGAAATGTTGAATACTCATACTCTTCCTTTTT CAATATTATTGAAGCATTTATCAGGGTTATTGTCTCATGAGCGGATACATATTTGAATGTATTTAGAAAAATAAACAAATAGGGGTTCCGCGCACAT TTCCCCGAAAAGTGCCACCTGACGTCTAAGAAACCATTATTATCATGACATTAACCTATAAAAATAGGCGTATCACGAGGCCCTTTCGTC

Total size $=3582$

NheI + ECORI [NEB4] $=2665+917$

PVuII $[$ NEB2] $=2364+826+392$

EagI + PStI [NEB3] $=3083+499$

Product of 1st round of PCR for $\mathrm{N}$-term His-tagged mCherry

ACCCACGCGCATGTCGTAAAAAGCACCCAAGTGAGCAAGGGCGAGGAGGATAACATGGCCATCATCAAGGAGTTCATGCGCTTCAAGGTGCACATGG AGGGCTCCGTGAACGGCCACGAGTTCGAGATCGAGGGCGAGGGCGAGGGCCGCCCCTACGAGGGCACCCAGACCGCCAAGCTGAAGGTGACCAAGGG TGGCCCCCTGCCCTTCGCCTGGGACATCCTGTCCCCTCAGTTCATGTACGGCTCCAAGGCCTACGTGAAGCACCCCGCCGACATCCCCGACTACTTG AAGCTGTCCTTCCCCGAGGGCTTCAAGTGGGAGCGCGTGATGAACTTCGAGGACGGCGGCGTGGTGACCGTGACCCAGGACTCCTCCCTGCAGGACG GCGAGTTCATCTACAAGGTGAAGCTGCGCGGCACCAACTTCCCCTCCGACGGCCCCGTAATGCAGAAGAAGACCATGGGCTGGGAGGCCTCCTCCGA GCGGATGTACCCCGAGGACGGCGCCCTGAAGGGCGAGATCAAGCAGAGGCTGAAGCTGAAGGACGGCGGCCACTACGACGCTGAGGTCAAGACCACC TACAAGGCCAAGAAGCCCGTGCAGCTGCCCGGCGCCTACAACGTCAACATCAAGTTGGACATCACCTCCCACAACGAGGACTACACCATCGTGGAAC AGTACGAACGCGCCGAGGGCCGCCACTCCACCGGCGGCATGGACGAGCTGTACAAGTAATAACTAACTAACCAAG

Product of 2nd (\& 3rd) round of PCR for $\mathrm{N}$-term His-tagged mCherry ATGATATCTCGAGCGGCCGCTAGCTAATACGACTCACTATAGGGAGACCACAACGGTTTCCCTCTAGAAATAATTTTGTTTAACTTTAAGAAGGAGA TAAACAATGAAACATCATCACCATCACCACTCGACCCACGCGCATGTCGTAAAAAGCACCCAAGTGAGCAAGGGCGAGGAGGATAACATGGCCATCA TCAAGGAGTTCATGCGCTTCAAGGTGCACATGGAGGGCTCCGTGAACGGCCACGAGTTCGAGATCGAGGGCGAGGGCGAGGGCCGCCCCTACGAGGG CACCCAGACCGCCAAGCTGAAGGTGACCAAGGGTGGCCCCCTGCCCTTCGCCTGGGACATCCTGTCCCCTCAGTTCATGTACGGCTCCAAGGCCTAC GTGAAGCACCCCGCCGACATCCCCGACTACTTGAAGCTGTCCTTCCCCGAGGGCTTCAAGTGGGAGCGCGTGATGAACTTCGAGGACGGCGGCGTGG TGACCGTGACCCAGGACTCCTCCCTGCAGGACGGCGAGTTCATCTACAAGGTGAAGCTGCGCGGCACCAACTTCCCCTCCGACGGCCCCGTAATGCA GAAGAAGACCATGGGCTGGGAGGCCTCCTCCGAGCGGATGTACCCCGAGGACGGCGCCCTGAAGGGCGAGATCAAGCAGAGGCTGAAGCTGAAGGAC GGCGGCCACTACGACGCTGAGGTCAAGACCACCTACAAGGCCAAGAAGCCCGTGCAGCTGCCCGGCGCCTACAACGTCAACATCAAGTTGGACATCA CCTCCCACAACGAGGACTACACCATCGTGGAACAGTACGAACGCGCCGAGGGCCGCCACTCCACCGGCGGCATGGACGAGCTGTACAAGTAATAACT AACTAACCAAGATCTGTACCCCTTGGGGCCTCTAAACGGGTCTTGAGGGGTTTTTTGGATCCGAATTCACCGGTGATATCAT

$\begin{array}{lll}\text { yellow } & \text { EcoRI } & G^{\wedge} \text { AATTC } \\ \text { green } & \text { BamHI } & G^{\wedge} \text { GATCC } \\ \text { red } & \text { EcoRV } & \text { GAT^ATC } \\ \text { purple } & \text { NheI } & G^{\wedge} \text { CTAGC } \\ \text { grey } & \text { XhoI } & \text { C }^{\wedge} \text { TCGAG } \\ \text { turquoise } & \text { PvuII } & \text { CAG^CTG } \\ \text { blue } & \text { EagI } & C^{\wedge} \text { GGCCG } \\ \text { teal } & \text { PstI } & C^{\prime} G C A \wedge G\end{array}$

NheI + ECoRI digest of $\mathrm{N}$-term His-tagged mCherry

CTAGCTAATACGACTCACTATAGGGAGACCACAACGGTTTCCCTCTAGAAATAATTTTGTTTAACTTTAAGAAGGAGATAAACAATGAAACATCATC ACCATCACCACTCGACCCACGCGCATGTCGTAAAAAGCACCCAAGTGAGCAAGGGCGAGGAGGATAACATGGCCATCATCAAGGAGTTCATGCGCTT 
CAAGGTGCACATGGAGGGCTCCGTGAACGGCCACGAGTTCGAGATCGAGGGCGAGGGCGAGGGCCGCCCCTACGAGGGCACCCAGACCGCCAAGCTG AAGGTGACCAAGGGTGGCCCCCTGCCCTTCGCCTGGGACATCCTGTCCCCTCAGTTCATGTACGGCTCCAAGGCCTACGTGAAGCACCCCGCCGACA TCCCCGACTACTTGAAGCTGTCCTTCCCCGAGGGCTTCAAGTGGGAGCGCGTGATGAACTTCGAGGACGGCGGCGTGGTGACCGTGACCCAGGACTC CTCCCTGCAGGACGGCGAGTTCATCTACAAGGTGAAGCTGCGCGGCACCAACTTCCCCTCCGACGGCCCCGTAATGCAGAAGAAGACCATGGGCTGG GAGGCCTCCTCCGAGCGGATGTACCCCGAGGACGGCGCCCTGAAGGGCGAGATCAAGCAGAGGCTGAAGCTGAAGGACGGCGGCCACTACGACGCTG AGGTCAAGACCACCTACAAGGCCAAGAAGCCCGTGCAGCTGCCCGGCGCCTACAACGTCAACATCAAGTTGGACATCACCTCCCACAACGAGGACTA CACCATCGTGGAACAGTACGAACGCGCCGAGGGCCGCCACTCCACCGGCGGCATGGACGAGCTGTACAAGTAATAACTAACTAACCAAGATCTGTAC CCCTTGGGGCCTCTAAACGGGTCTTGAGGGGTTTTTTGGATCCG

\section{NheI + EcoRI frag of $\mathrm{N}$-term His-tagged mCherry cloned in pIX3.0}

TCGCGCGTTTCGGTGATGACGGTGAAAACCTCTGACACATGCAGCTCCCGGAGACGGTCACAGCTTGTCTGTAAGCGGATGCCGGGAGCAGACAAGC CCGTCAGGGCGCGTCAGCGGGTGTTGGCGGGTGTCGGGGCTGGCTTAACTATGCGGCATCAGAGCAGATTGTACTGAGAGTGCACCATATGCGGTGT GAAATACCGCACAGATGCGTAAGGAGAAAATACCGCATCAGGCGCCATTCGCCATTCAGGCTGCGCAACTGTTGGGAAGGGCGATCGGTGCGGGCCT CTTCGCTATTACGCCAGCTGGCGAAAGGGGGATGTGCTGCAAGGCGATTAAGTTGGGTAACGCCAGGGTTTTCCCAGTCACGACGTTGTAAAACGAC GGCCAGTGCCAAGCTTTCTCGAGCGGCCGCTAGCTAATACGACTCACTATAGGGAGACCACAACGGTTTCCCTCTAGAAATAATTTTGTTTAACTTT AAGAAGGAGATAAACAATGAAACATCATCACCATCACCACTCGACCCACGCGCATGTCGTAAAAAGCACCCAAGTGAGCAAGGGCGAGGAGGATAAC ATGGCCATCATCAAGGAGTTCATGCGCTTCAAGGTGCACATGGAGGGCTCCGTGAACGGCCACGAGTTCGAGATCGAGGGCGAGGGCGAGGGCCGCC CCTACGAGGGCACCCAGACCGCCAAGCTGAAGGTGACCAAGGGTGGCCCCCTGCCCTTCGCCTGGGACATCCTGTCCCCTCAGTTCATGTACGGCTC CAAGGCCTACGTGAAGCACCCCGCCGACATCCCCGACTACTTGAAGCTGTCCTTCCCCGAGGGCTTCAAGTGGGAGCGCGTGATGAACTTCGAGGAC GGCGGCGTGGTGACCGTGACCCAGGACTCCTCCCTGCAGGACGGCGAGTTCATCTACAAGGTGAAGCTGCGCGGCACCAACTTCCCCTCCGACGGCC CCGTAATGCAGAAGAAGACCATGGGCTGGGAGGCCTCCTCCGAGCGGATGTACCCCGAGGACGGCGCCCTGAAGGGCGAGATCAAGCAGAGGCTGAA GCTGAAGGACGGCGGCCACTACGACGCTGAGGTCAAGACCACCTACAAGGCCAAGAAGCCCGTGCAGCTGCCCGGCGCCTACAACGTCAACATCAAG TTGGACATCACCTCCCACAACGAGGACTACACCATCGTGGAACAGTACGAACGCGCCGAGGGCCGCCACTCCACCGGCGGCATGGACGAGCTGTACA AGTAATAACTAACTAACCAAGATCTGTACCCCTTGGGGCCTCTAAACGGGTCTTGAGGGGTTTTTTGGATCCGAATTCACCGGTGCAATTCGTAATC ATGTCATAGCTGTTTCCTGTGTGAAATTGTTATCCGCTCACAATTCCACACAACATACGAGCCGGAAGCATAAAGTGTAAAGCCTGGGGTGCCTAAT GAGTGAGCTAACTCACATTAATTGCGTTGCGCTCACTGCCCGCTTTCCAGTCGGGAAACCTGTCGTGCCAGCTGCATTAATGAATCGGCCAACGCGC GGGGAGAGGCGGTTTGCGTATTGGGCGCTCTTCCGCTTCCTCGCTCACTGACTCGCTGCGCTCGGTCGTTCGGCTGCGGCGAGCGGTATCAGCTCAC TCAAAGGCGGTAATACGGTTATCCACAGAATCAGGGGATAACGCAGGAAAGAACATGTGAGCAAAAGGCCAGCAAAAGGCCAGGAACCGTAAAAAGG CCGCGTTGCTGGCGTTTTTCCATAGGCTCCGCCCCCCTGACGAGCATCACAAAAATCGACGCTCAAGTCAGAGGTGGCGAAACCCGACAGGACTATA AAGATACCAGGCGTTTCCCCCTGGAAGCTCCCTCGTGCGCTCTCCTGTTCCGACCCTGCCGCTTACCGGATACCTGTCCGCCTTTCTCCCTTCGGGA AGCGTGGCGCTTTCTCATAGCTCACGCTGTAGGTATCTCAGTTCGGTGTAGGTCGTTCGCTCCAAGCTGGGCTGTGTGCACGAACCCCCCGTTCAGC CCGACCGCTGCGCCTTATCCGGTAACTATCGTCTTGAGTCCAACCCGGTAAGACACGACTTATCGCCACTGGCAGCAGCCACTGGTAACAGGATTAG CAGAGCGAGGTATGTAGGCGGTGCTACAGAGTTCTTGAAGTGGTGGCCTAACTACGGCTACACTAGAAGAACAGTATTTGGTATCTGCGCTCTGCTG AAGCCAGTTACCTTCGGAAAAAGAGTTGGTAGCTCTTGATCCGGCAAACAAACCACCGCTGGTAGCGGTGGTTTTTTTGTTTGCAAGCAGCAGATTA CGCGCAGAAAAAAAGGATCTCAAGAAGATCCTTTGATCTTTTCTACGGGGTCTGACGCTCAGTGGAACGAAAACTCACGTTAAGGGATTTTGGTCAT GAGATTATCAAAAAGGATCTTCACCTAGATCCTTTTAAATTAAAAATGAAGTTTTAAATCAATCTAAAGTATATATGAGTAAACTTGGTCTGACAGT TACCAATGCTTAATCAGTGAGGCACCTATCTCAGCGATCTGTCTATTTCGTTCATCCATAGTTGCCTGACTCCCCGTCGTGTAGATAACTACGATAC GGGAGGGCTTACCATCTGGCCCCAGTGCTGCAATGATACCGCGAGACCCACGCTCACCGGCTCCAGATTTATCAGCAATAAACCAGCCAGCCGGAAG GGCCGAGCGCAGAAGTGGTCCTGCAACTTTATCCGCCTCCATCCAGTCTATTAATTGTTGCCGGGAAGCTAGAGTAAGTAGTTCGCCAGTTAATAGT TTGCGCAACGTTGTTGCCATTGCTACAGGCATCGTGGTGTCACGCTCGTCGTTTGGTATGGCTTCATTCAGCTCCGGTTCCCAACGATCAAGGCGAG TTACATGATCCCCCATGTTGTGCAAAAAAGCGGTTAGCTCCTTCGGTCCTCCGATCGTTGTCAGAAGTAAGTTGGCCGCAGTGTTATCACTCATGGT TATGGCAGCACTGCATAATTCTCTTACTGTCATGCCATCCGTAAGATGCTTTTCTGTGACTGGTGAGTACTCAACCAAGTCATTCTGAGAATAGTGT ATGCGGCGACCGAGTTGCTCTTGCCCGGCGTCAATACGGGATAATACCGCGCCACATAGCAGAACTTTAAAAGTGCTCATCATTGGAAAACGTTCTT CGGGGCGAAAACTCTCAAGGATCTTACCGCTGTTGAGATCCAGTTCGATGTAACCCACTCGTGCACCCAACTGATCTTCAGCATCTTTTACTTTCAC CAGCGTTTCTGGGTGAGCAAAAACAGGAAGGCAAAATGCCGCAAAAAAGGGAATAAGGGCGACACGGAAATGTTGAATACTCATACTCTTCCTTTTT CAATATTATTGAAGCATTTATCAGGGTTATTGTCTCATGAGCGGATACATATTTGAATGTATTTAGAAAAATAAACAAATAGGGGTTCCGCGCACAT TTCCCCGAAAAGTGCCACCTGACGTCTAAGAAACCATTATTATCATGACATTAACCTATAAAAATAGGCGTATCACGAGGCCCTTTCGTC

Total size $=3582$

NheI + ECORI [NEB4] $=2665+917$

PVUII $[$ NEB2 $]=2364+826+392$

EagI + PStI [NEB3] $=3083+499$

Hundreds of clones of each construct were recovered in E. coli, and eight of each were characterized through an extensive series of diagnostic restriction digests (8 combinations of restriction enzymes, 10 gels). We confirmed that all of the cloned constructs, with the possible exception of pIX3.0-6xHis-mOrange \#6, were correct.

We pooled the correct clones for each construct and confirmed that the products supported expression of fluorescent protein in the Qiagen EasyXpress Linear Template Kit Plus. We then compared this kit head-to-head with the Promega S30 T7 High-Yield Protein Expression System (cat\# L1115), which is advertised as being particularly adept at high-yield recombinant protein 
expression. Positive and negative controls were included, and we attempted expression at $37^{\circ} \mathrm{C}$ for $1 \mathrm{hr}$ and $30^{\circ} \mathrm{C}$ for $5 \mathrm{hr}$. The unpurified reaction products were analyzed by SDS-PAGE and Coomassie Blue staining, and we also checked protein function by measuring fluorescence at red wavelengths using a fluorimeter. Our initial results suggested that the Promega kit used at $30^{\circ} \mathrm{C}$ for $5 \mathrm{hr}$ gave us the best yield; in the following figure you can see a decent band in the last lane (next to the MW standard) of 30-35 kDa, the positive control ran (poorly) at $36 \mathrm{kDa}$ as expected.

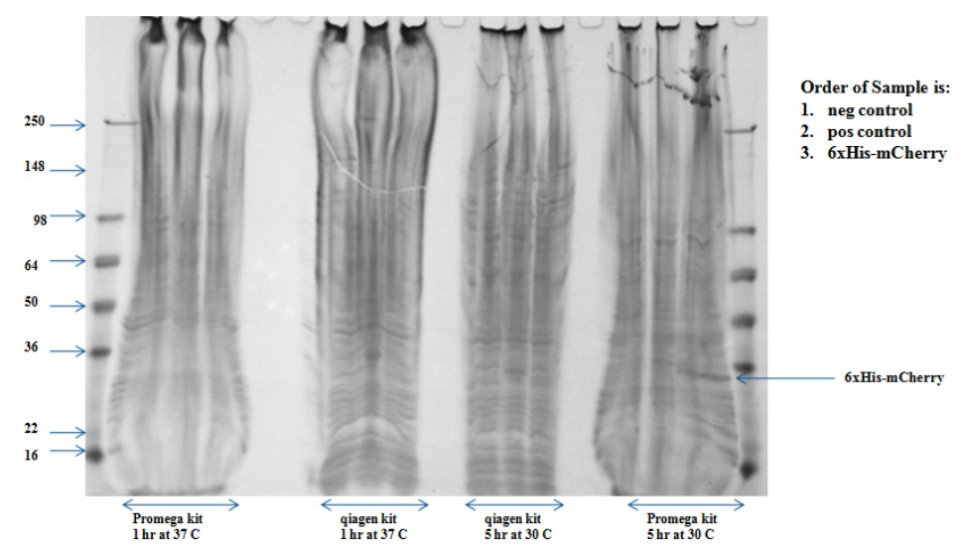

Figure 2-1.

We then used both kits at $30^{\circ} \mathrm{C}$ for $5 \mathrm{hr}$, and also attempted to purify the recombinant protein from the reaction lysates using Ni-NTA magnetic agarose beads (Qiagen cat\# 36111).

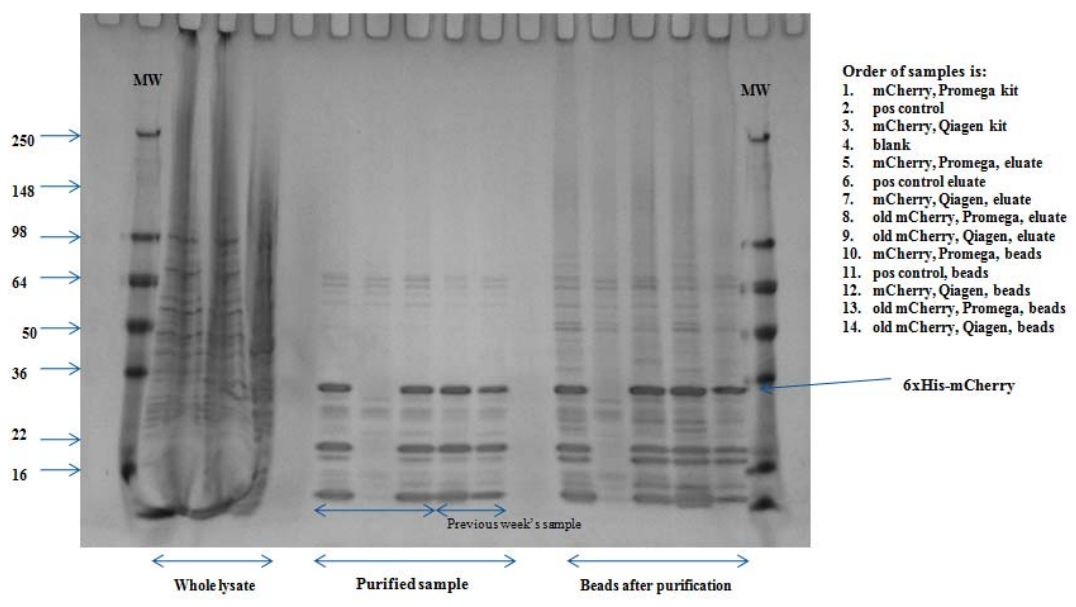

Figure 2-2.

We found that while the $\sim 34 \mathrm{kDa}$ 6xHis-mCherry band was much more easily detected upon purification, so too were a series of smaller bands - perhaps degradation products? Also unexpected was the fact that a significant amount of the purified proteins failed to elute from the beads. 
At this point we conducted a long series of experiments in which we attempted to optimize the expression and purification of 6xHis-mCherry using the Qiagen kit. We varied template concentration, expression temperature and time, concentration of imadazole in the elution buffer, and elution temperature and time. Eventually we settled on a standard protocol that consistently yielded abundant and pure 6xHis-mCherry that was brightly fluorescent (and therefore functional). The "degradation products" were consistently observed, but they did not appear to vary much from prep to prep, and there was no obvious impact on levels of full-length 6xHismCherry or its fluorescence.

\subsubsection{In vitro expression of 10xHis-hTLR4ed and 10xHis-hMD2.}

Once we were confident in our standardized in vitro expression and purification protocol we turned our attention from fluorescent proteins and instead focused on TLR4 and MD2. The first step was to obtain constructs which support in vitro expression. In the case of TLR4 it was necessary to generate a new construct. Our strategy took into consideration the requirements for inserting a fluorescent protein (Kusabi Orange, mCherry, or eGFP) gene into the construct at a later date. The following is the strategy for construct generation, including insertion of the eGFP gene:

atgatatctcga cggccgctagctaatacgactcactatagggagaccacaacggtttccctctagaaataattttgtttaactttaagaaggaga taaaca tgGTGAGCAAGGGCGAGGAGCTGTTCACCGGGGTGGTGCCCATCCTGGTCGAGCTGGACGGCGACGTAAACGGCCACAAGTTCAGCGTGT CCGGCGAGGGCGAGGGCGATGCCACCTACGGCAAGCTGACCCTGAAGTTCATCTGCACCACCGGCAAGCTGCCCGTGCCCTGGCCCACCCTCGTGAC CACCCTGACCTACGGCGTGCAGTGCTTCAGCCGCTACCCCGACCACATGAAGCAGCACGACTTCTTCAAGTCCGCCATGCCCGAAGGCTACGTCCAG GAGCGCACCATCTTCTTCAAGGACGACGGCAACTACAAGACCCGCGCCGAGGTGAAGTTCGAGGGCGACACCCTGGTGAACCGCATCGAGCTGAAGG GCATCGACTTCAAGGAGGACGGCAACATCCTGGGGCACAAGCTGGAGTACAACTACAACAGCCACAACGTCTATATCATGGCCGACAAGCAGAAGAA CGGCATCAAGGTGAACTTCAAGATCCGCCACAACATCGAGGACGGCAGCGTGCAGCTCGCCGACCACTACCAGCAGAACACCCCCATCGGCGACGGC CCCGTGCTGCTGCCCGACAACCACTACCTGAGCACCCAGTCCGCCCTGAGCAAAGACCCCAACGAGAAGCGCGATCACATGGTCCTGCTGGAGTTCG TGACCGCCGCCGGGATCACTCTCGGCATGGACGAGCTGTACAAGTCCGGACTCAGATCTCGAGCTCAAGCTTCGAATTCTGCAGTCGACGGTACCGC GGGCCCGGGATCCACCGGATCTAGAGAAAGCTGGGAGCCCTGCGTGGAGGTGGTTCCTAATATTACTTATCAATGCATGGAGCTGAATTTCTACAAA ATCCCCGACAACCTCCCCTTCTCAACCAAGAACCTGGACCTGAGCTTTAATCCCCTGAGGCATTTAGGCAGCTATAGCTTCTTCAGTTTCCCAGAAC TGCAGGTGCTGGATTTATCCAGGTGTGAAATCCAGACAATTGAAGATGGGGCATATCAGAGCCTAAGCCACCTCTCTACCTTAATATTGACAGGAAA CCCCATCCAGAGTTTAGCCCTGGGAGCCTTTTCTGGACTATCAAGTTTACAGAAGCTGGTGGCTGTGGAGACAAATCTAGCATCTCTAGAGAACTTC CCCATTGGACATCTCAAAACTTTGAAAGAACTTAATGTGGCTCACAATCTTATCCAATCTTTCAAATTACCTGAGTATTTTTCTAATCTGACCAATC TAGAGCACTTGGACCTTTCCAGCAACAAGATTCAAAGTATTTATTGCACAGACTTGCGGGTTCTACATCAAATGCCCCTACTCAATCTCTCTTTAGA CCTGTCCCTGAACCCTATGAACTTTATCCAACCAGGTGCATTTAAAGAAATTAGGCTTCATAAGCTGACTTTAAGAAATAATTTTGATAGTTTAAAT GTAATGAAAACTTGTATTCAAGGTCTGGCTGGTTTAGAAGTCCATCGTTTGGTTCTGGGAGAATTTAGAAATGAAGGAAACTTGGAAAAGTTTGACA AATCTGCTCTAGAGGGCCTGTGCAATTTGACCATTGAAGAATTCCGATTAGCATACTTAGACTACTACCTCGATGATATTATTGACTTATTTAATTG TTTGACAAATGTTTCTTCATTTTCCCTGGTGAGTGTGACTATTGAAAGGGTAAAAGACTTTTCTTATAATTTCGGATGGCAACATTTAGAATTAGTT AACTGTAAATTTGGACAGTTTCCCACATTGAAACTCAAATCTCTCAAAAGGCTTACTTTCACTTCCAACAAAGGTGGGAATGCTTTTTCAGAAGTTG ATCTACCAAGCCTTGAGTTTCTAGATCTCAGTAGAAATGGCTTGAGTTTCAAAGGTTGCTGTTCTCAAAGTGATTTTGGGACAACCAGCCTAAAGTA TTTAGATCTGAGCTTCAATGGTGTTATTACCATGAGTTCAAACTTCTTGGGCTTAGAACAACTAGAACATCTGGATTTCCAGCATTCCAATTTGAAA CAAATGAGTGAGTTTTCAGTATTCCTATCACTCAGAAACCTCATTTACCTTGACATTTCTCATACTCACACCAGAGTTGCTTTCAATGGCATCTTCA ATGGCTTGTCCAGTCTCGAAGTCTTGAAAATGGCTGGCAATTCTTTCCAGGAAAACTTCCTTCCAGATATCTTCACAGAGCTGAGAAACTTGACCTT CCTGGACCTCTCTCAGTGTCAACTGGAGCAGTTGTCTCCAACAGCATTTAACTCACTCTCCAGTCTTCAGGTACTAAATATGAGCCACAACAACTTC TTTTCATTGGATACGTTTCCTTATAAGTGTCTGAACTCCCTCCAGGTTCTTGATTACAGTCTCAATCACATAATGACTTCCAAAAAACAGGAACTAC AGCATTTTCCAAGTAGTCTAGCTTTCTTAAATCTTACTCAGAATGACTTTGCTTGTACTTGTGAACACCAGAGTTTCCTGCAATGGATCAAGGACCA GAGGCAGCTCTTGGTGGAAGTTGAACGAATGGAATGTGCAACACCTTCAGATAAGCAGGGCATGCCTGTGCTGAGTTTGAATATCACCTGTCAGATG AATAAGtggggtcaccaccatcaccatcatCACCATCATCACtaataactaactaaccaagatctgtacccettgggg cetctaaacgggtcttgag gggttttt gaattcaccggtgatatcat

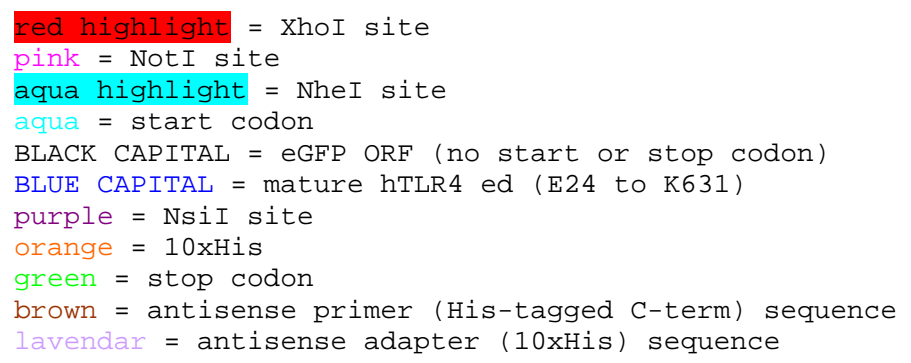


bold italics $=$ to be synthesized

grey highlight $=$ BamHI site

hTLR4ed-10xHis sense primer (order): agaaggagataaacaatgGAAAGCTGGGAGCCCTGCGTG

hTLR4ed-10xHis antisense primer (order): tggtgatggtggtgacccCaCTTATTCATCTGACAGGTGATATTC

no-tag sense adapter (comes with kit):

atgatatctcgag cggccgctagctaatacgactcactatagggagaccacaacggtttccctctagaaataattttgtttaactttaagaaggaga taaca

10xHis antisense adapter (order):

atgatatcaccggtgaattcggatccaaaaaaccctcaagacccgtttagaggccccaaggggtacagatcttggttagttagttattaGTGATGA TGGTGatgatggtgatggtggtgacccca

1) Generate hTLR4ed-10xHis fragment ( 2000bp) using kit

2) Clone hTLR4ed-10xHis XhoI-BamHI fragment ( 200๑bp) into pIX3.0 XhoI-BamHI vector ( $2700 \mathrm{bp})$. - use BamHI-HF + XhoI, NEB4 + BSA

3) Synthesize eGFP ORF with flanking regions (bold italics); 948bp.

4) Digest plasmid ( 4700bp) and synthesized fragment (948bp) with NotI + NsiI.

- use NotI (not HF) + NSiI, NEB3 + BSA

- can use NheI (not HF) instead of NotI, but double-digest conditions are suboptimal

5) Ligate synthesized fragment into plasmid, replacing the $150 \mathrm{bp}$ piece that drops out upon double-digestion.

We recovered a number of clones that showed correct band patterns in a number of diagnostic restriction digests. We did not go ahead with insertion of fluorescent protein genes right away; instead, we decided to obtain or generate a His-tagged MD2 contruct and then test the constructs individually and together in in vitro expression assays. We mapped out a strategy for generating a 6xHis-MD2 construct:

$\underline{\text { hMD2 }}$

source $=$ pEF-BOS-MD2 $[$ Int Immunol 13:1595] or MD2-FLAG [JBC 277:23427]

ATGTTACCATTTCTGTTTTTTTCCACCCTGTTTTCTTCCATATTTACTGAAGCTCAGAAGCAGTATTGGGTCTGCAACTCATCCGATGCAAGTATTT CATACACCTACTGTGATAAAATGCAATACCCAATTTCAATTAATGTTAACCCCTGTATAGAATTGAAAAGATCCAAAGGATTATTGCACATTTTCTA CATTCCAAGGAGAGATTTAAAGCAATTATATTTCAATCTCTATATAACTGTCAACACCATGAATCTTCCAAAGCGCAAAGAAGTTATTTGCCGAGGA TCTGATGACGATTACTCTTTTTGCAGAGCTCTGAAGGGAGAGACTGTGAATACAACAATATCATTCTCCTTCAAGGGAATAAAATTTTCTAAGGGAA AATACAAATGTGTTGTTGAAGCTATTTCTGGGAGCCCAGAAGAAATGCTCTTTTGCTTGGAGTTTGTCATCCTACACCAACCTAATTCAAATTAG

green = natural start codon

red = natural stop codon

pink = signal peptide

$\underline{\text { underlined }}=\underline{\text { primer } \text { sequences }}$

\section{N-term 6xHis}

sense primer (hMD2 $\mathbf{N}-\mathbf{6} \mathbf{6 H} \mathbf{A})$ : ACCCACGCGCATGTCGTAAAAAGCACCCAACAGAAGCAGTATTGGGTCT

aqua $=$ Qiagen-designed

blue = mature hMD2 $\mathrm{N}$-term

antisense primer (hMD2 $\mathbf{N}-\mathbf{6 x H} \mathbf{B}):$ CTTGGTTAGTTAGTTATTAATTTGAATTAGGTTGGTGTAG

aqua $=$ Qiagen-designed

orange = synthetic stop codon (reverse-complement)

purple = hMD2 C-term (reverse-complement)

\section{C-term 6xHis}


aqua $=$ Qiagen-designed

brown = synthetic start codon

blue = mature hMD2 $\mathrm{N}$-term

antisense primer (hMD2 $\mathbf{C - 6 x H}$ B): TGGTGATGGTGGTGACCCCAATTTGAATTAGGTTGGTGTAG

aqua $=$ Qiagen-designed

purple = hMD2 C-term (reverse-complement)

Before we started down the path of generating a new 6xHis-MD2 construct, however, we found that GeneCopoeia sells both 6xHis-hMD2 and hMD2-6xHis constructs (EX-U0747-BO1 and EX-U0747-B31, respectively). We bought these, and tried them out for in vitro expression reactions, in parallel with 10xHis-hTLR4ed.

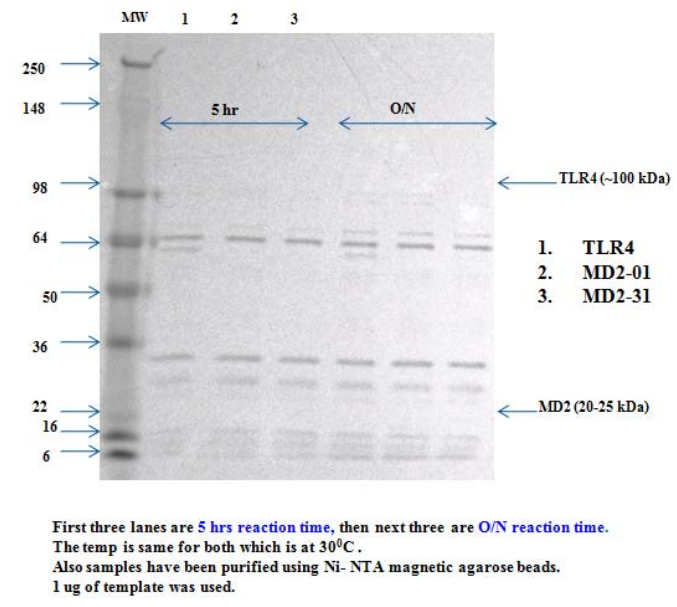

Figure 2-3.

We expected the TLR4 protein to run at $\sim 100 \mathrm{kDa}$ and the MD2 protein to run at 20-25 $\mathrm{kDa}$. We saw nothing that looked at all convincing. Follow-up experiments in which we varied template concentration, expression temperature and time, and elution temperature all showed similar results. The reactions were working - parallel reactions supported robust 6xHis-mCherry expression.

Further tweaking of reaction conditions seemed pointless, given that we had no positive signal to improve upon. Instead, we decided to attempt expression in intact E. coli. The pIX3.0-10xHishTLR4ed plasmid was introduced into E. coli strain BL21, and the cells grown in large batch culture at $37 \mu \mathrm{C}$ to mid-log phase before inducing with IPTG for $0-5 \mathrm{hr}$. The cells were then lysed, and the membranes pelleted by centrifugation. The "soluble protein" was in the supernatant, the "concentrated protein" was first ethanol precipitated and then resuspended in a smaller volume. The "insoluable protein" was associated with the cell pellet. In this experiment none of the fractions convincingly showed a 100 kDa band, or indeed anything that looked like it could be derived from 10xHis-hTLR4ed. 


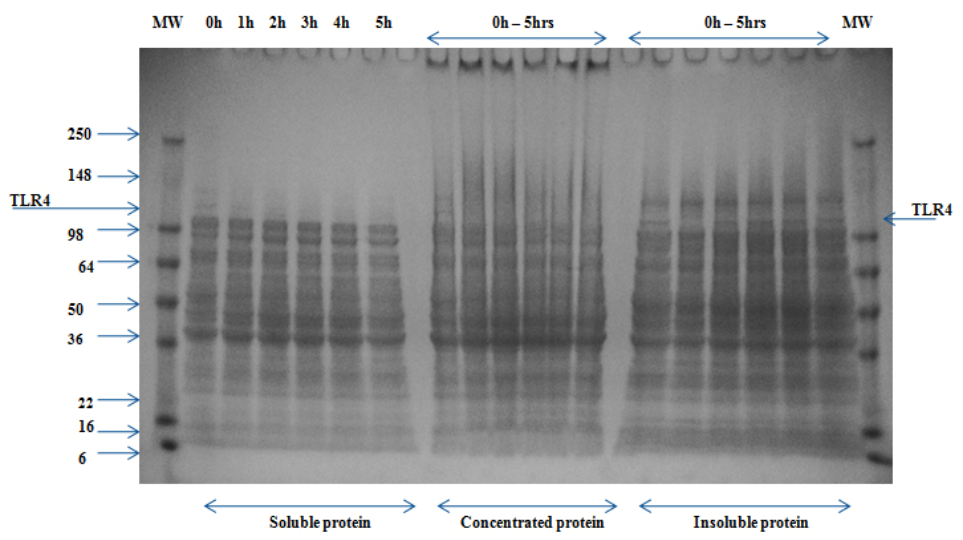

Figure 2-4.

We repeated the 10xHis-hTLR4ed expression reactions, carrying out 6xHis-hMD2 ("MD2-01") and hMD2-6xHis ("MD2-31") expression reactions in parallel, and analyzed all of the products (total lysates) by SDS-PAGE.

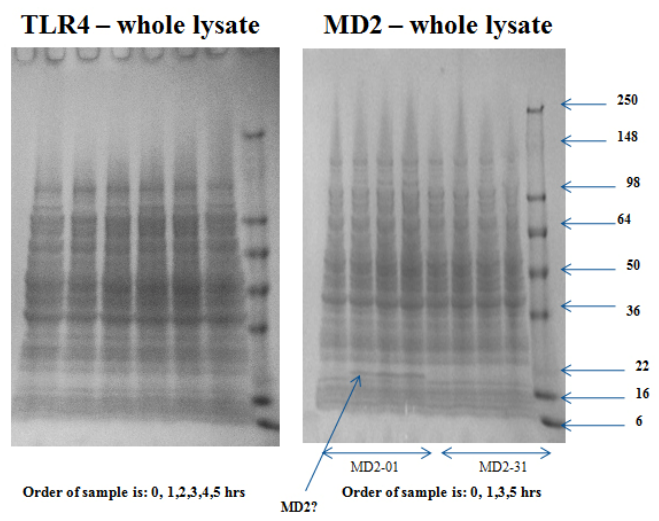

Figure 2-5.

Again, there were no signs at all of a specific 10xHis-hTLR4ed signal. However, the 6xHishMD2 reactions showed a convincing band of $\sim 20 \mathrm{kDa}$ that grew in intensity over time, as expression progressed. This was the first indication of in-house expression of a TLR4 complex subunit, and we quickly followed up with an experiment in which we attempted to purify the reaction products using Ni-NTA beads. 

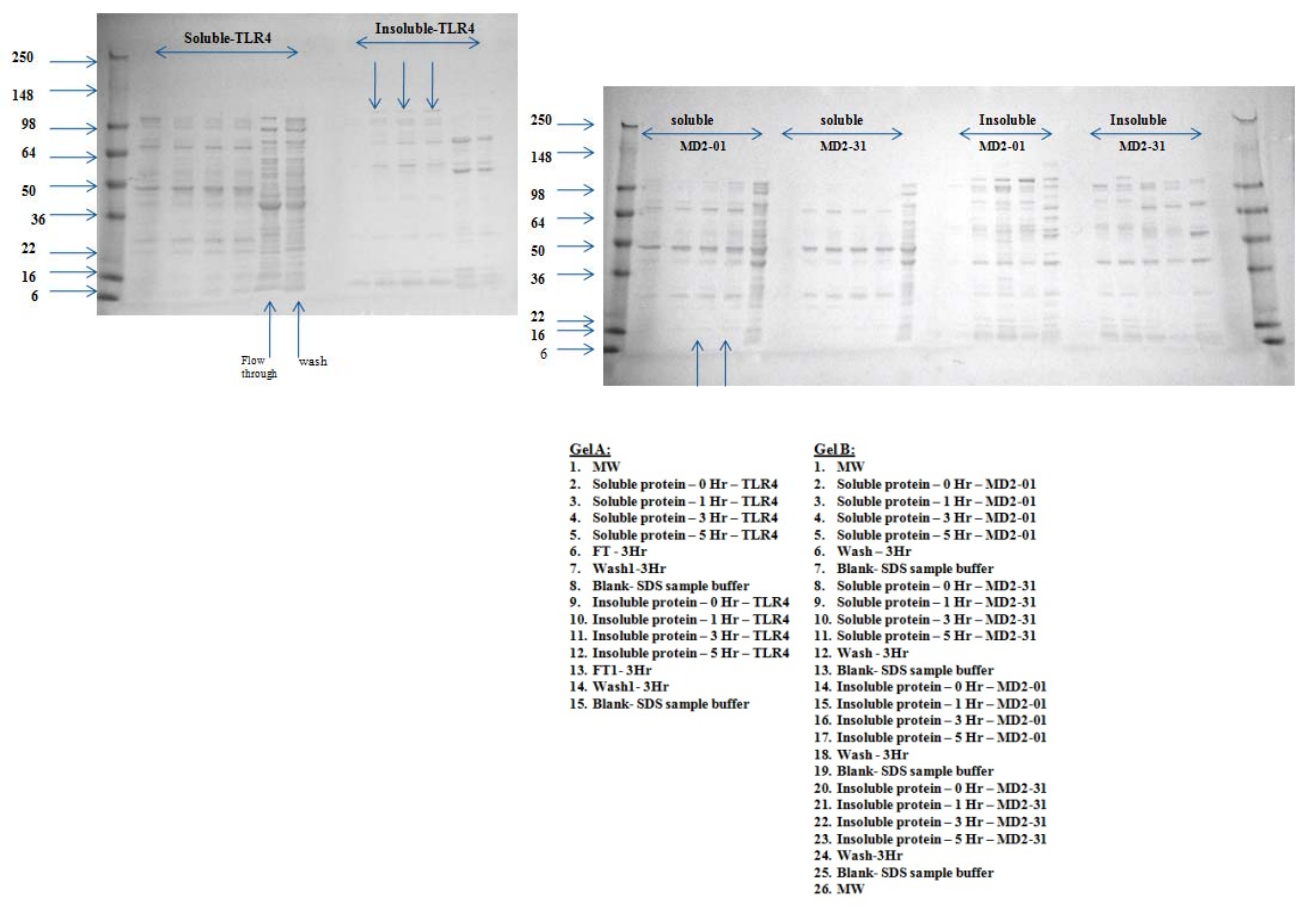

Figure 2-6.

In this experiment some seemingly specific but weak $100 \mathrm{kDa}$ bands appeared in the lanes analyzing the insoluble fractions of the 10xHis-hTLR4ed reactions. Similarly, we again observed specific $20 \mathrm{kDa}$ bands in the lanes analyzing the soluble fractions of the 6xHis-hMD2 reactions; the bands were weaker this time, despite the additional step of Ni-NTA bead mediated purification, suggesting that purification of 6xHis-hMD2 was inefficient.

By this point the evidence suggested that cell-free expression of His-tagged TLR4 and MD2 was marginal at best. The seemingly specific bands were very weak; not much of a signal to guide optimization of expression and purification conditions. In an attempt to boost the signal for this purpose, we carried out Western analysis of the products from our cell-free expression reactions. After SDS-PAGE the proteins were transferred to a nitrocellulose membrane, probed with an anti-His-tag antibody, and detected via chemiluminescence. 


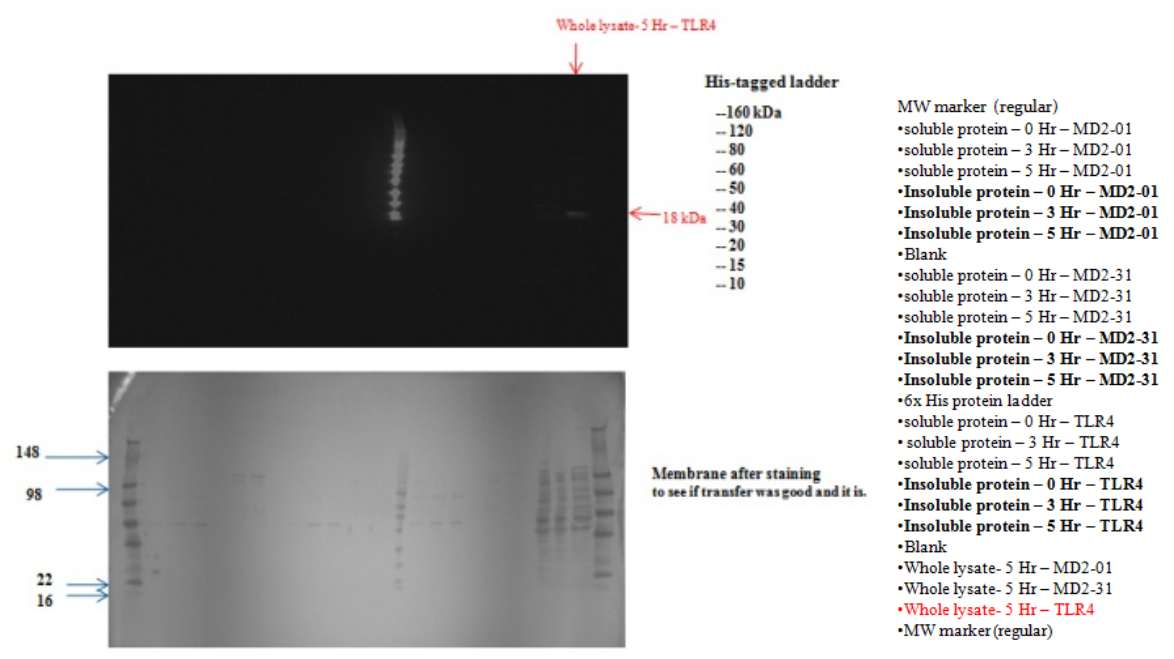

Figure 2-7.

While the His-tagged MW standard was easily detected, the only other noticeable signal on the membrane was an $18 \mathrm{kDa}$ band in a lane loaded with untreated lysate from a $5 \mathrm{hr} 10 \mathrm{xHis}-$ hTLR4ed expression reaction. We expected an $\sim 100 \mathrm{kDa}$ band in that lane; perhaps the $\sim 18 \mathrm{kDa}$ band was a degradation product? In any case, Western analysis did not improve our TLR4- and MD2-specific signals in the way that we had hoped, and the path forward with cell-free reactions did not look promising at this point.

\subsection{E. coli expression of recombinant proteins}

\subsubsection{Expression of 10xHis-hTLR4ed and 6xHis-hMD2}

Given our limited success with in vitro expression of 10xHis-hTLR4ed and 6xHis-hMD2, we next turned to expression in E. coli. These experiments were more onerous, but it seemed the only path forward because in general in vitro expression yields are at least an order of magnitude lower than E. coli expression yields, and our protein-specific signals were weak at best.

E. coli strain BL21 was transformed with the 10xHis-hTLR4ed and 6xHis-hMD2 contructs, and a single purified clone from each transformation was used to inoculate a starting culture of $5 \mathrm{ml}$ of $\mathrm{LB}+$ ampicillin. After growth at $37^{\circ} \mathrm{C}$ for $\sim 8 \mathrm{hr}$, a $200 \mathrm{ml} \mathrm{LB}+$ ampicillin culture was inoculated and grown at $37^{\circ} \mathrm{C}$ for $\sim 16 \mathrm{hr}$. In turn, this culture was used to inoculate a $2 \mathrm{~L} \mathrm{LB}+$ ampicillin culture that was grown at $37^{\circ} \mathrm{C}$ for $\sim 5 \mathrm{hr}$ to late-log phase, then spiked with IPTG and grown for an additional 5 hrs. Cells were harvested by centrifugation, washed twice with cold PBS, lysed through sonication, and separated into soluble (supernatant) vs insoluble (pellet) fractions. Ni-NTA beads were added to each fraction and incubated with gentle mixing at $4^{\circ} \mathrm{C}$ for $\sim 16 \mathrm{hr}$ (a procedure recommended for improved harvesting of His-tagged proteins from $E$. coli lysate). Bead-bound proteins were washed and then eluated with imadazole, using the protocol developed for our in vitro expression experiments. The eluates were analyzed by SDSPAGE and Coomassie Blue staining. 


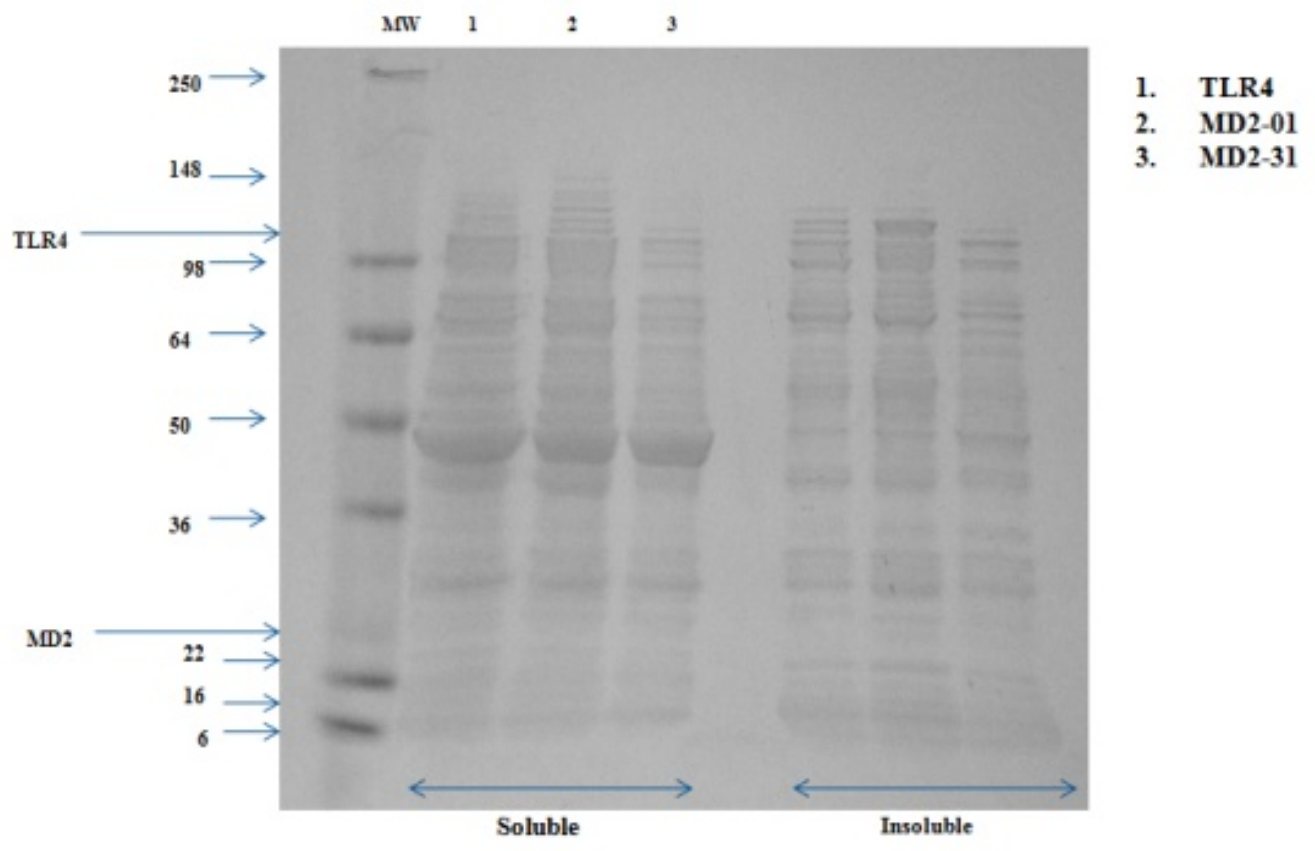

Figure 2-8.

We observed no specific bands corresponding to the expected sizes of 10xHis-hTLR4ed ( 100 $\mathrm{kDa})$ or 6xHis-hMD2 (20-25 kDa).

\subsection{Summary of recombinant protein expression work}

We generated constructs and optimized in vitro expression and purification conditions for robust production of multiple recombinant His-tagged fluorescent proteins. These fluorescent proteins served as useful standards for our work with Cu-impregnated lipid bilayers and microscopy. However, the comparable constructs and protocols that we developed for in vitro production of recombinant His-tagged TLR4 or MD2 were not effective, and expression in E. coli also failed. At this time it is unclear where the problem lies. We could return to the very weak and inconsistent signals observed in the in vitro expression reactions and further attempt optimization, but we're not optimistic about that path. Additional work with E. coli expression might be warranted; we did not do much in the way of varying conditions in that expression system, so there may be a lot of room for improvement. However, before starting down that road it might be advisable to generate some new constructs and carry them forward in parallel, in case the ones we have been using simply will not support robust expression of their respective recombinant proteins, regardless of reaction conditions. On the face of it there is no rational reason to suspect as much, other than the fact that thus far they have failed to work under the conditions we have tested. But given the amount of time, effort, and resources required for revisiting these experiments, it might be best to do so with a suite of newly-generated expression constructs. 


\section{STRUCTURAL STUDIES}

\subsection{NR studies of structure of membrane-bound protein}

NR is one of very few methods that can resolve structural details of membrane-associated proteins in physiological conditions, and may be unique in its ability to directly resolve details of the full membrane-bound protein structure, in contrast to techniques that probe only labeled residues or secondary structural elements. NR involves measuring the ratio of reflected to incident intensity as a function of momentum transfer $q_{z}=4 \pi \sin \theta / \lambda$, where $q_{z}$ is the angle of incidence with respect to the plane of the membrane and $\lambda$ is the wavelength (38). The form of this curve is determined by the in-plane averaged scattering length density (SLD) profile normal to the surface. The SLD is directly related to the atomic composition and the density. Therefore, for a protein bound to a planar lipid membrane, NR determines the in-plane averaged distribution of amino acid residues normal to the membrane, and is sensitive to any changes in that distribution.

Several platforms are used to study conformational changes of proteins interacting with lipid membranes by these methods. Each has advantages and limitations, and the information from different platforms must sometimes be combined to obtain the most complete picture. The Langmuir monolayer is a platform that is simple to implement and for which the data analysis is straightforward due to the fact that only a single lipid layer is present. However, this method requires a relatively large volume of solution $(18 \mathrm{ml})$. This was important in the present study as the protein was available in short supply and was very expensive. Lipid bilayers supported directly on a silicon substrate constitutes a second platform. This has the advantage of requiring much less solution ( $3 \mathrm{ml}$ ) but data analysis and interpretation are substantially complicated by the presence of two lipid layers and the possibility of lipids exchanging between the two layers.

The NR data were analyzed using the Ga_refl program based on the optical matrix method. Ga_refl is available at www.ncnr.nist.gov. Simultaneous fits of the NR data were performed for multiple data sets involving different contrast conditions, and for multiple data sets at different stages of a single adsorption run (for example lipids only, with adsorbed protein, and after subphase exchange). Simultaneous analysis allowed particular characteristics to be maintained constant for all the fits, such as the SLD of the subphase and specific characteristics of the lipid layers. In all cases, the fits included only the minimum number of layers for the protein that were required to achieve a good fit to the data. In the Ga_refl program the roughness parameter is the full width at half maximum (FWHM $=2.35 \mathrm{~s}$, where $\mathrm{s}$ is the standard deviation) of a Gaussian distribution and was constrained in the fitting to be less than the smallest thickness of the two adjacent layers.

Fitting reflectivity data results in defining a family of SLD curves that are consistent with the data. The uncertainty in the fitted profiles was determined by a Monte Carlo resampling procedure in which a large number (1000) of statistically independent sets of reflectivity data were created from the original data set and the error bars from the counting statistics. Each set of reflectivity data was analyzed using the fitting procedure described above. The result is a range of values for each fit parameter that is consistent with the statistics of the original data. The fit of 
each reflectivity set was initiated with random values for the fit parameters. This method has been reported in detail elsewhere (41).

\subsubsection{Method Development}

\section{Synthesis of deuterated DSIDA.}

In this work the soluble portion of the TLR4 receptor in complex with MD2 was bound to lipid membranes using the interaction of a His tag with divalent $\mathrm{Cu}^{2+}$ ions chelated in the headgroups of the synthetic lipids 1,2-distearylglycero-3-triethyleneoxideiminodiacetic acid (DSIDA) and 1,2-dioleoylglycero-3-triethyleneoxideiminodiacetic acid (DOIDA). Synthesizing deuterated DSIDA was critical to extend the $q_{z}$ range of the neutron reflectivity data and achieve the highest possible resolution. The synthesis of DSIDA has been described previously. Deuterated DSIDA $\left(\mathrm{d}_{70}\right.$-DSIDA) was synthesized using a similar procedure starting with perdeuterated octadecanoic acid (Larodan). NMR results for d-DSIDA are as follows: $\quad\left[{ }^{1} \mathrm{H}\right.$ NMR $\left(\mathrm{CDCl}_{3}\right) \delta 3.69$ (m, 4H, $\mathrm{NCH}_{2} \mathrm{CO}-$ ), $3.64-3.41$ (m, 21H, $-\mathrm{CH}_{2} \mathrm{O}-$ ), 3.05 (br s, $2 \mathrm{H},-\mathrm{NCH}_{2}$ ), 1.21 (m, 3H, $-\mathrm{CH}-$ ). ${ }^{13} \mathrm{C}$ $\left(\mathrm{CDCl}_{3}\right) \delta 172.61,104.77,77.77,71.70,71.28,70.59,70.48,70.40,70.21,70.17,58.68,55.81$, 44.77, 28.65. IR (KBr) 3439, 2878, 2193, 2088, 1736, 1637, 1370, 1256, $1118 \mathrm{~cm}^{-1}$. Anal. calc. for $\mathrm{C}_{49} \mathrm{H}_{27} \mathrm{NO}_{9} \mathrm{D}_{70}$ : C, 64.34; H, 10.69; N, 1.53. Found: C, 64.65; H, 10.61; N, 1.51.].

\section{Langmuir monolayers.}

In our initial work to bind His-tagged proteins to DSIDA/ $\mathrm{Cu}^{2+}$-containing membranes we made the discovered that $5 \mathrm{mM}$ of the reducing agent DTT reduced $\mathrm{Cu}^{2+}$ to $\mathrm{Cu}^{+}$and inhibited binding of His tag. As mentioned further below we also discovered that the same DTT concentration led to conformational changes in MD2 due to reduction of internal disulfide bonds. Subsequent work was performed without any reducing agent in the buffer.

In order to reduce the amount of protein required in the measurement, we explored use of polyethylene glycol to inhibit binding of protein to tubing and Teflon surfaces of trough. This was successful in that the amount of protein required for membrane binding was reduced, and the PEG did not affect the membrane or the conformation of the membrane-bound protein in a way that could be detected by neutron reflectivity or surface tension measurements.

In subsequent work we determined the optimal way of introducing His-tagged proteins in order to reduce the amount of protein required. We discovered that injecting the protein directly underneath the monolayer rather than through the circulation system greatly increased the loading of protein onto the membrane for a given concentration. This is most likely due to greater loss of protein when added through the circulation system due to binding of protein to the surfaces of the tubing and Teflon trough.

We also determined the optimal amount of deuteration in the lipids for use with fully deuterated protein and for use with fully protonated protein. Use of fully deuterated lipids extends the $q_{z}$ range to the highest possible values, but at the expense of a reduced sensitivity to the protein. 
With protonated protein, use of $30-40 \%$ deuterated lipids resulted in a good balance of strong sensitivity to the protein and reasonably good $q_{z}$ range. However, with deuterated protein, good sensitivity to the protein occurred even with fully deuterated lipids.

\section{Supported lipid bilayers.}

Due to delays in in-house expression of the soluble portion of TLR4 (His-tagged ectodomain of TLR4 $4_{\text {ECD }}$ ) and the high cost of purchasing large quantities of the His-tagged TLR4/MD2 and His-tagged MD2, substantial effort was made to perform NR at the solid-liquid interface rather than the liquid-air interface (Langmuir monolayers) in order to reduce the amount of protein needed by $>80 \%$. However, several preliminary steps were required in order to study TLR4/MD2 at the solid-liquid interface due to the need to deposit bilayers with high fractions of the His-tag binding lipid DSIDA. Following difficulties with liposome fusion, we learned how to deposit bilayers containing DSIDA and PC lipids by the Langmuir-Blodgett method. In the process we discovered that the tails of DSIDA and the PC lipid must have the same length for optimal deposition. We then explored deposition by the Langmuir-Blodgett/Langmuir-Schaefer method. This provided improved control over lipid composition and coverage. However, through the use of isotopically label lipids we discovered that the lipids exchanged between the two layers during the reflectivity measurement, complicating the interpretation of the data. We found that this could be minimized by using DSPC $\left(\mathrm{T}_{\text {gel }}=55 \mathrm{C}\right)$ rather than $\mathrm{DPPC}\left(\mathrm{T}_{\text {gel }}=41 \mathrm{C}\right)$ for the monolayer proximal to the substrate. Measurements were made with both His-tagged TLR4/MD2 and also with His-tagged d-Nef (discussed further below in 3.1.4). The latter avoided the high cost of using TLR/MD2 during the troubleshooting and benefited from the availability of fully deuterated Nef. These data were important in their own right, and were used to support several NIH proposals and resulted in a major publication.

\subsubsection{Results: MD2}

Initial measurements indicated a large conformational change in MD2, consistent with one of the hypothesized models for TLR4 dimerization. However, this was later revealed to be due to the presence of a reducing agent in the buffer. Three disulfide bonds are present in MD2 that are integral to its folded structure. (Ohto Science 2007) Experiments repeated in the absence of a reducing agent showed no change in conformation. Results for MD2 bound to DSIDA/ $\mathrm{Cu}^{2+} / \mathrm{DPPG}$ are shown in Fig 3-1. After exchanging the subphase with buffer the data indicated a monomeric dimension. This is important as MD2 is known to form disulfide linked dimers and in the absence of a reducing agent the protein bound to the membrane could have been largely the dimer. We note that dimerization in the plane of the membrane cannot be detected by NR, and so dimmers could still be present. Yet we observe only a monomeric dimension normal to the membrane which strongly suggests that only monomeric MD2 is bound to the membrane at the concentrations used in this study. We assume that due to more rapid diffusion the monomeric form quickly fills up the surface sites $\left(\mathrm{Cu}^{2+}\right.$ ions). 

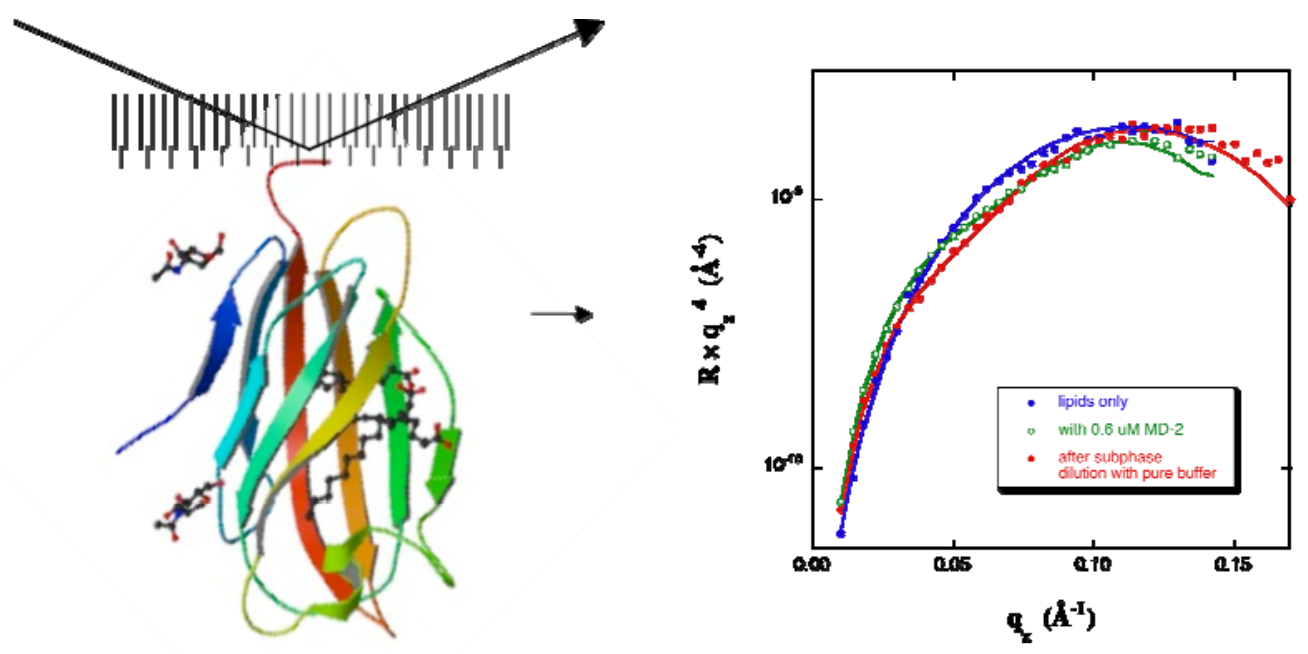

MD2
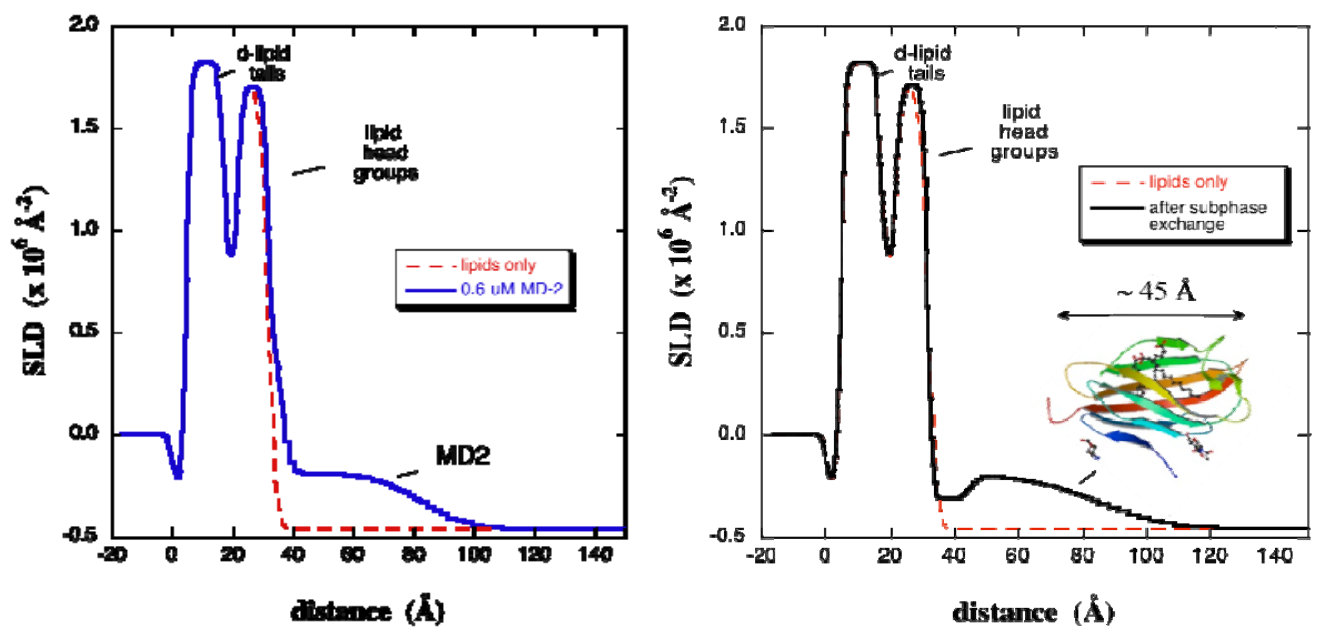

Figure 3-1. NR data for His-tagged MD2 bound to 35\% DSIDA + 65\% d-DPPG and SLD profiles resulting from the fitting analysis.

\subsubsection{Results: TLR4/MD2}

Further NR work involved the ectodomain of TLR4 (His-tagged TLR4 ECD) with bound MD2 purchased from R\&D. Due to delays in in-house expression of these proteins and the high cost of purchasing large quantities of the proteins, it was decided to perform NR at the solid-liquid interface rather than the liquid-air in order to reduce the amount of protein needed by $>80 \%$. Several preliminary steps were required in order to study TLR4/MD2 at the solid-liquid interface due to the need to deposit bilayers with high fractions of the His-tag binding lipid DSIDA. Following difficulties with liposome fusion, we learned how to deposit bilayers containing 
DSIDA and PC lipids by the Langmuir-Blodgett method. In the process we discovered that the tails of DSIDA and the PC lipid must have the same length for optimal deposition. We then explored deposition by the Langmuir-Blodget/Langmuir-Schaefer method. This provided improved control over lipid composition and coverage. However, through the use of isotopically label lipids we discovered that the lipids exchanged between the two layers during the reflectivity measurement, complicating the interpretation of the data. We found that this could be minimized by using DSPC $\left(T_{\text {gel }}=55 \mathrm{C}\right)$ rather than DPPC $\left(T_{\text {gel }}=41 \mathrm{C}\right)$ as the monolayer proximal to the substrate, but not entirely eliminated. Results are shown in Fig 3-2. Also shown in this figure is a plot of calculated reflectivity from the hypothesized dimerized structure shown. A series of calculations for different dimeric structures was planned but halted when the crystal structure of the dimerized complex was published. In Fig 3-2 the presence of the membrane bound protein is evident in the data but the effect is very weak and extends only to $\mathrm{Q}=0.06 \AA^{-1}$, providing only very low resolution. Further work was not pursued due to the publication of the crystal structure of the dimerized complex.
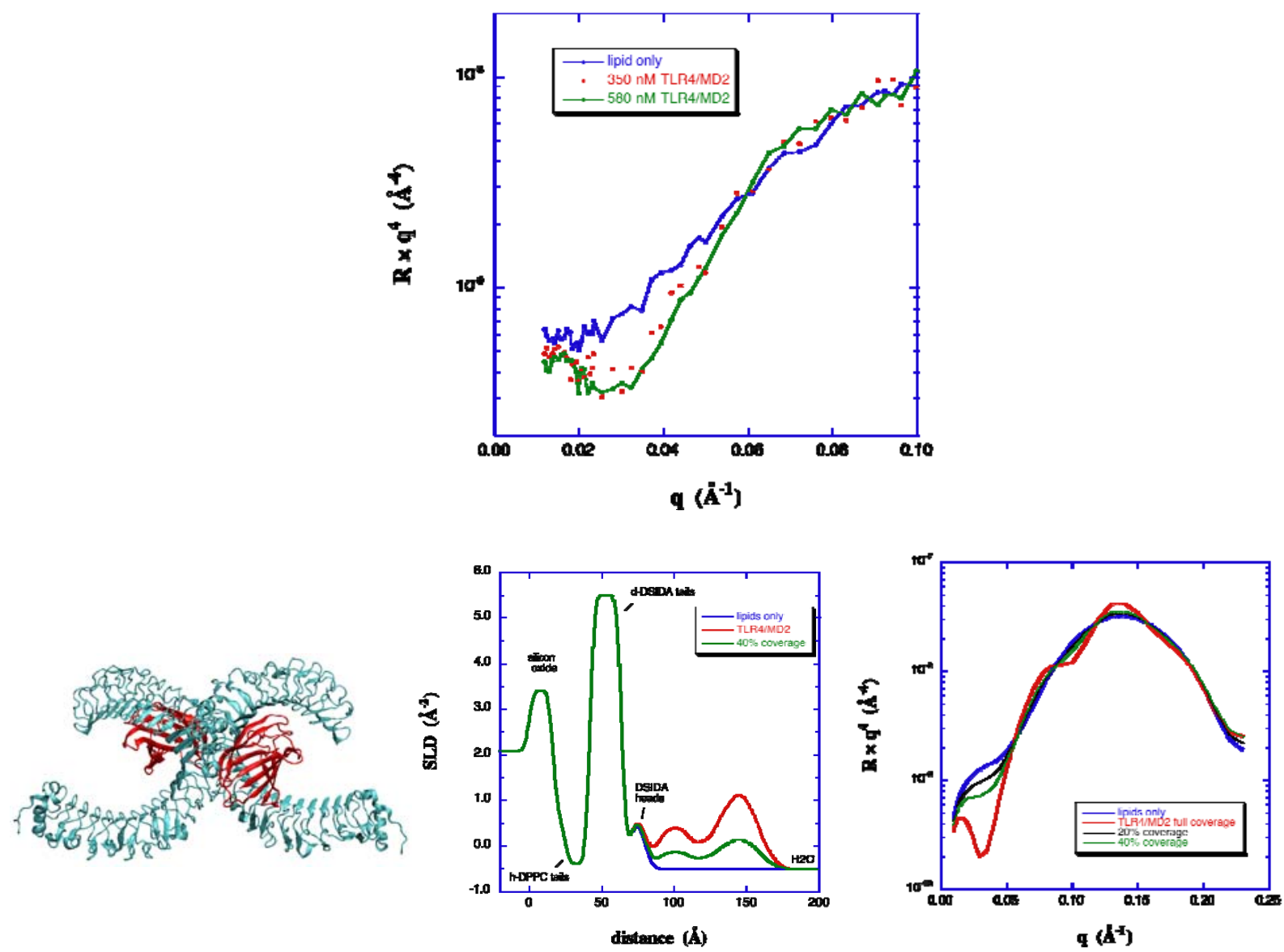

Figure 3-2. NR data for His-tagged TLR4/MD2 bound to 35\% DSIDA + 65\% d-DPPG. Also shown are calculated SLD profiles and reflectivity for the hypothesized dimeric structure on the left. 


\subsubsection{Results: HIV Nef}

HIV Nef was used in the method development stage as it was available in large quantities and with most of the labile protons replaced with deuterium. TLR4/MD2 was available commercially but not in a deuterium enriched form. Also, the commercial product was very expensive $\$ 300 / 50$ ug, and large fractions of a mg was required for each experiment. Therefore Nef was used to develop and optimize the NR measurements.

Nef is an HIV-1 accessory protein that directly contributes to AIDS progression. Nef is myristoylated on the $\mathrm{N}$-terminus, associates with membranes, and may undergo a transition from a solution conformation to a membrane-associated conformation. It has been hypothesized that conformational rearrangement enables membrane-associated Nef to interact with cellular proteins. Despite its medical relevance, there is no direct information about the conformation of membrane-bound Nef. In this work we used neutron reflection (NR) to reveal the first details of the conformation of membrane-bound Nef.

In addition to aiding in the development of the method, important results were obtained that led to a major publication and supported several grant applications, including a successful proposal to NIH. Both good resolution and a strong neutron scattering signal were achieved with the use of deuterated lipids and d-Nef against an $\mathrm{H}_{2} \mathrm{O}$ subphase, as evident in Fig 3-3 where the effect of the protein is evident in the data out to $\mathrm{q}=0.23 \AA^{-1}$, the mechanical limit of this spectometer. The reflectivity data and area per molecule data both indicated insertion of residues into the membrane upon binding of Nef through the N-terminal His tag. When interpreted in light of prior studies, this strongly suggests that a portion of the N-terminal arm inserts into the membrane. Upon binding through the N-terminus and insertion, the core domain of Nef is located directly against the lipid headgroups and forms a layer approximately $40 \AA$ thick. This is important as binding sites of the core domain appear to be blocked in this conformation. Binding of Nef to the same membrane also occurred at a much lower level in absence of the interaction through the His tag, however, in that case no insertion of residues into the membrane was detected. We conclude that binding through the $\mathrm{N}$-terminus contributes to $\mathrm{N}$-terminal arm insertion. Further details can be found in the ref .
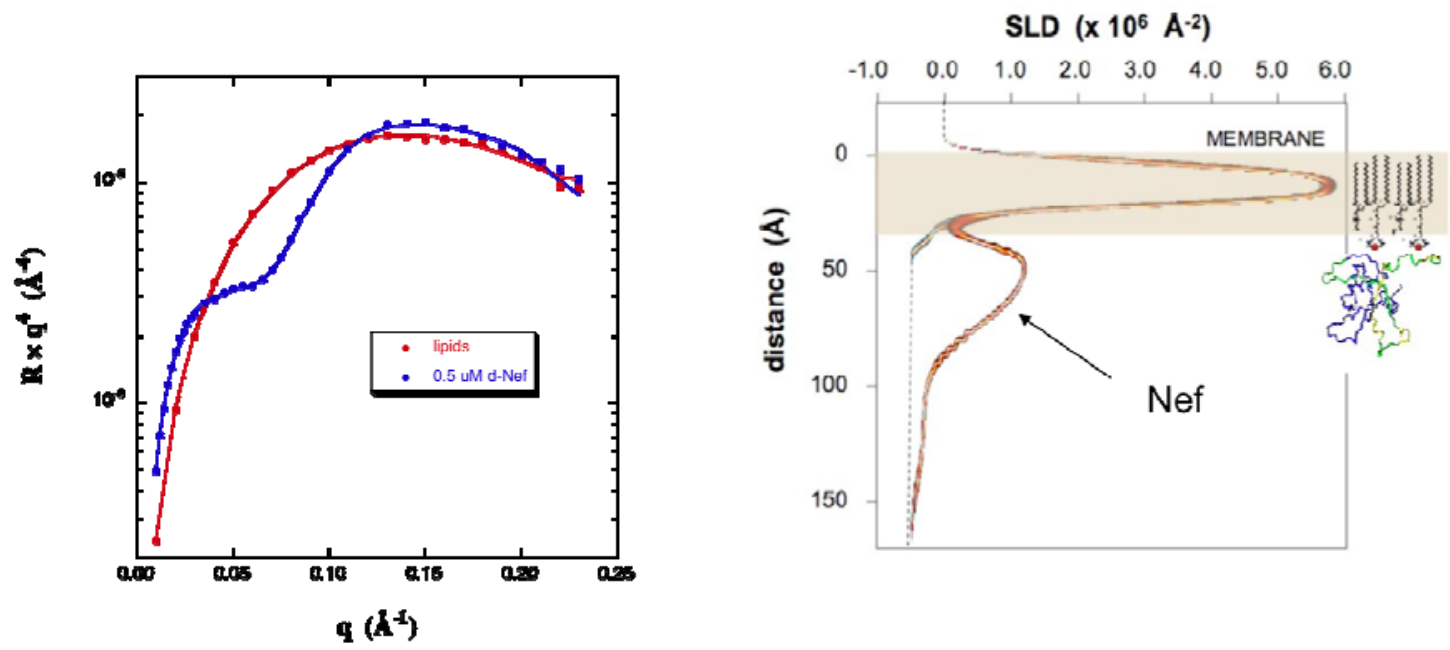

Figure 3-3. NR data for His-tagged d-Nef bound to 35\% d-DSIDA + 65\% d-DPPC. 


\subsection{Electron Microscopy Imaging}

\subsubsection{Results: Negative stained EM images of soluble TLR4-MD2 complexes}

The TLR4-MD2 complex was purchased from R\&D Systems frozen at $6 \mathrm{mg} / \mathrm{ml}$ in PBS. The complex was thawed, diluted to $40 \mu \mathrm{g} / \mathrm{ml}$ and negatively stained with $2 \%$ uranyl acetate on EM grids with thin carbon support film (Sherman, Orlova et al. 1981; Sherman, Guenther et al. 2006).

Images were acquired on a JEM 2200FS electron microscope (JEOL) using slow-scan CCD camera (895, GATAN, Inc). Images of individual TLR4-MD2 particles were boxed out interactively using BOXER program (EMAN, (Ludtke, Baldwin et al. 1999)) and further processed in IMAGIC (van Heel, Harauz et al. 1996). Electron optics of an electron microscope due to its various aberrations (spherical aberration, astigmatism, defocus, limited spatial coherence, etc.) distorts images, that is why image processing in EM requires image restoration (contrast transfer function correction, CTF correction). Defocus was measured for each individual micrograph and CTF correction was performed in IMAGIC. Defocus values ranged from $-40 \mathrm{~nm}$ to $-90 \mathrm{~nm}$. 3178 images of TLR4-MD2 particles were boxed out. The images were found to be heterogeneous in size, larger size particles were discarded using MSA analysis and only particles of smaller size corresponding to molecular mass of the complex were used for further analysis. The particles of TLR4-MD2 complex were lying randomly on carbon support film, meaning the image ensemble would contain practically all different projections of them. A field of uranyl acetate stained TLR4-MD2 heterodimers is shown in Figure 3-4.

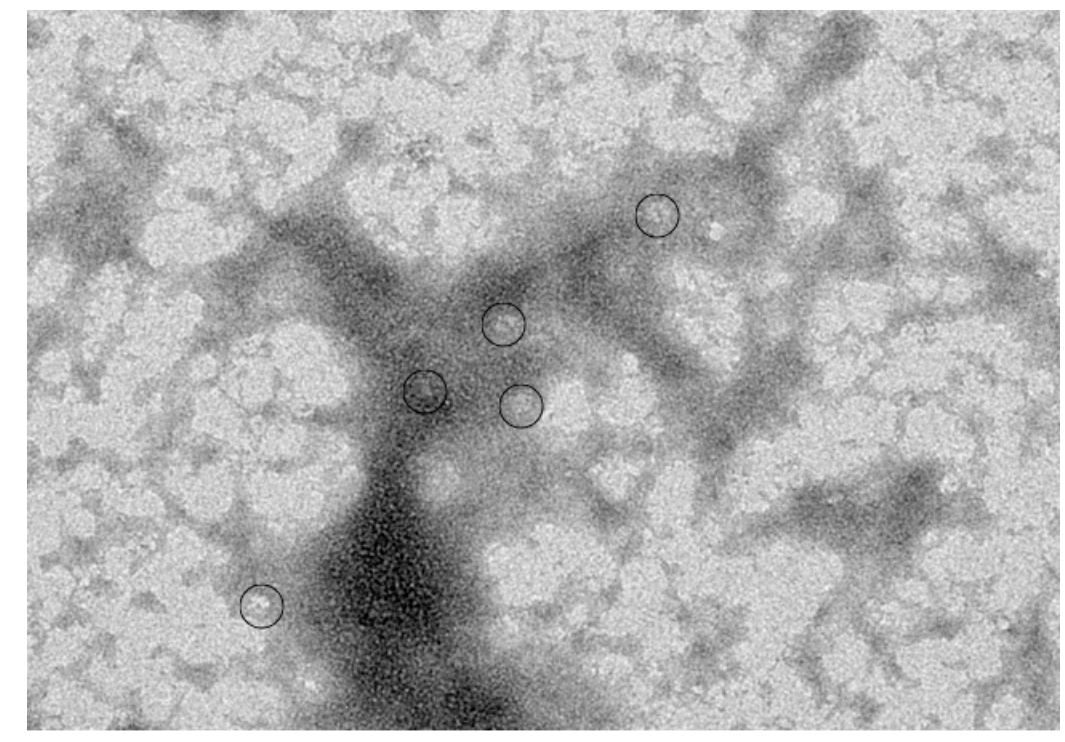

Figure 3-4. Negatively stained with 2\% uranyl acetate TLR4-MD2 heterodimer. Several particles in different orientations are encircled.

Individual images of the particles are extremely noisy; to reduce the noise images of TLR4-MD2 in similar orientations were aligned, classified, and finally averaged. For subsequent analysis 
class averages (characteristic views) were used. Their relative orientations were determined using Angular Reconstitution program from IMAGIC suite. Angular reconstitution uses common one-dimensional projections present in any of two images (projections) of the same threedimensional object; that allows for $a b$ initio determination of the orientations in space. After initial model was reconstructed, new alignment using multiple references was performed and new classes were obtained, their orientations were determined followed by new 3D reconstruction (Orlova, Sherman et al. 1999). After several iterations the process converged and the final reconstruction was calculated. Figure 3-5 shows selected characteristic views and corresponding reprojections of the 3D reconstruction. Figure 3-6 shows manual fitting of highresolution structure of TLR4-MD2 into 3D EM map.

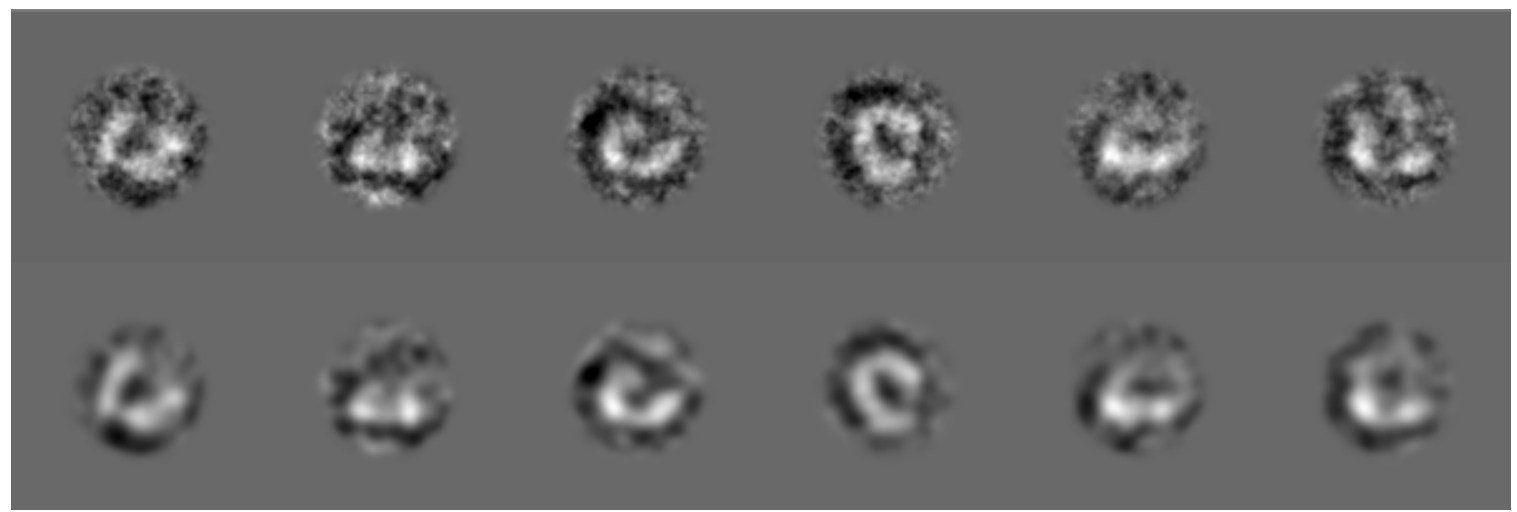

Figure 3-5. Upper row: selected characteristic views of TLR4-MD2 heterodimers negatively stained with uranyl acetate. Bottom row: 3D map reprojected in the orientations corresponding the characteristic views. Good matches between the rows showed correct orientation determination between the views.
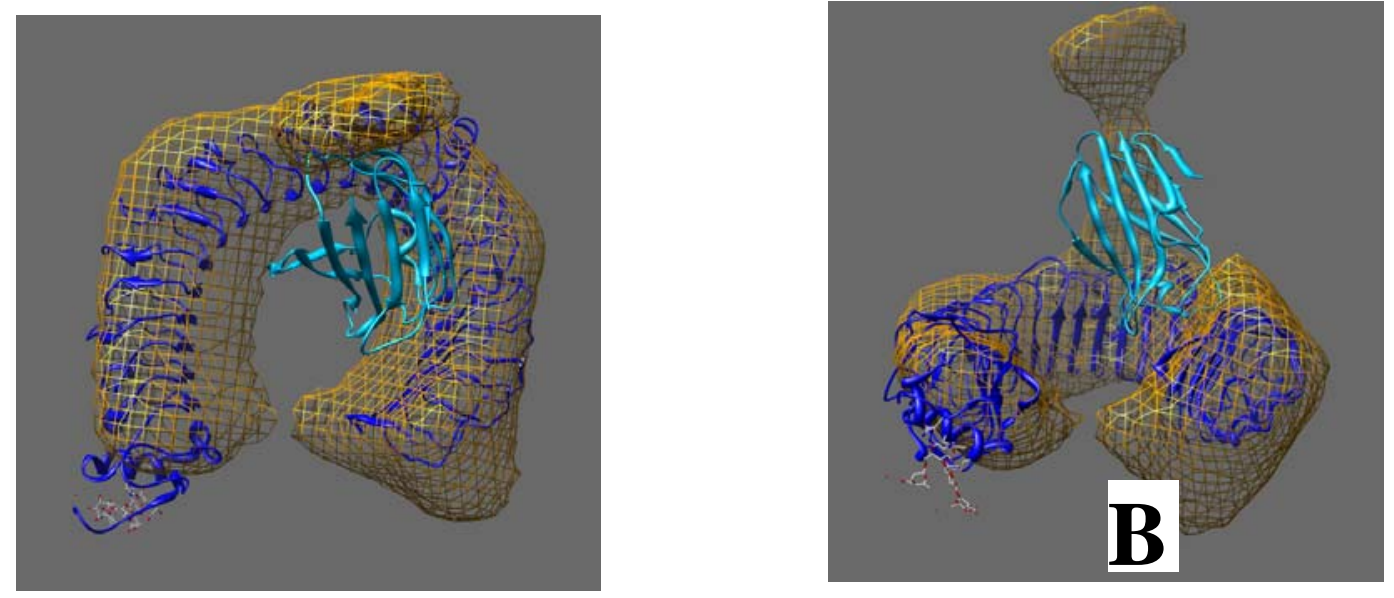

Figure 3-6. TLR4-MD2 dimer (blue-cyan ribbon) fitted into 3D EM map (golden mesh) 


\subsubsection{CryoEM images of soluble complexes of TLR4-MD2}

In the FY2008-2009 M. Sherman switched from imaging of negatively stained, dry preparations of TLR4/MD2 complex to cryo-electron microscopy of that complex. Negative staining (heavy metal salt staining), although dramatically increasing image contrast and preserving gross features of biological has limited applications in electron microscopy of macromolecules. It is a good starting technique allowing evaluation of feasibility of the EM studies of a particular type of the specimens, but could lead to significant distortions of the 3D architecture, specimen flattening on the support film during drying and structural changes owing to chemical interactions of the macromolecules with the negative stain. Therefore after first imaging and 3D reconstruction of TLR4/MD2 complex stained with uranyl acetate M. Sherman used more advanced cryoEM technique to preserve TLR4/MD2 complex in nearly native conformation. Imaging was also done with low dose technique that minimized electron irradiation of the specimen and allowed data collection essentially without radiation damages incurred by incident electrons in the microscope. Due to much lower radiation stability of the frozen sample compared to negative staining electron exposure was made at the 20 electrons/ $/ \AA^{2}$ dose, severe underexposure conditions with high shot electron noise. Having in mind inherently low image contrast of biological unstained specimens in electron microscopes that led to very low signal-tonoise $(\mathrm{S} / \mathrm{N})$ ratio of the order of $0.1-0.01$. To enhance signal and to suppress the noise cryoEM requires merging information from very large ensemble of images of individual molecules each of which is an identical chemical entity but possibly oriented differently in 3D space.

The imaging was impaired by hurricane Ike at the beginning of September 2008 that devastated the whole Galveston Island and UTMB in particular. Tragically, the electron microscopes in M. Sherman cryoEM Facility were immersed in about one foot of seawater for many hours, and were damaged beyond repair. UTMB is now in the process of restoring the Facility. Meanwhile M. Sherman has established agreements with the Baylor College of Medicine and the University of Texas Health Science Center, Houston, to use their facilities for cryoEM imaging.

TLR4/MD2 complex was flash frozen over holes in C-Flat grids (ProtoChips Co) using liquid ethane (cooled by liquid nitrogen) as the cryogen. The frozen girds were stored under liquid nitrogen and transferred to cryo-specimen holder at liquid nitrogen temperature. They were maintained at that temperature in the microscope during data acquisition so that the sample stayed vitrified all the time. 


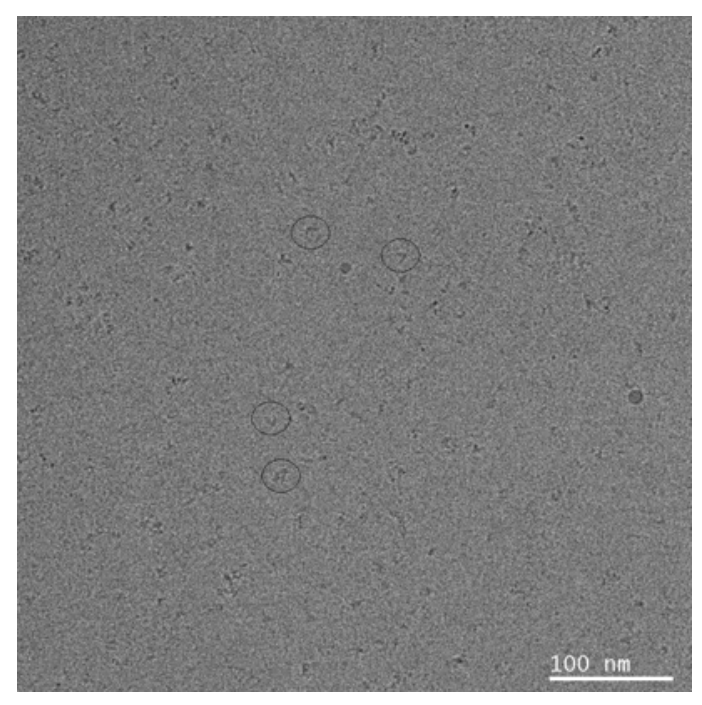

Figure 3-7. TLR4/MD2 resuspended in bufffer and plunge-frozen in liquid ethane. Several individual complexes are encircled.

More than 10,000 images of individual TLR4/MD2 complexes were acquired using slow-scan CCD detector in a JEOL 2010F cryo-electron microscope at Baylor College of Medicine (Houston). Images of individual TLR4/MD2 complexes were boxed out from CCD frames, aligned, classified, and their orientations were found. M. Sherman has observed heterogeneity in the images; along with full TLR4/MD2 complex there were separate TLR4 and MD2 particles present. That points to dynamic equilibrium between the complex and its components, or to disintegration of the complex. Certain amount of aggregation was observed in the images as well. The best images were used to calculate 3D reconstruction. The reconstruction accurately represents $3 \mathrm{D}$ organization of the complex (it is similar to the published Xray structure). 


\section{STUDIES OF LPS-INDUCED OLIGOMERIZATION}

To study LPS induced oligomerization, several chemotypes were initially used, including full $E$. coli LPS lipid A, lipid IVa, and E. coli LPS Ra mutant. However, eventually we focused on $E$. coli LPS Ra mutant following the work described in the report of the crystal structure (ref). Our goal was to develop methods to study LPS-induced dimerization for soluble TLR4/MD2 and also for TLR4/MD2- bound to lipid membranes. Much greater progress was made with the former than the later, as developing methods to probe LPS-induced dimerization for the membranebound receptor proved difficult.

\subsection{Soluble TLR4/MD2}

\subsubsection{Gels}

We used sodium dodecyl sulfate polyacrylamide gel electrophoesis (SDS-PAGE) to first determine the sample quality of the TLR4/MD2 product from R\&D. Then we investigated the use of native gels to detect the change in molecular weight as a signature of oligomerization. This method has the advantage of being relatively nonintrusive. However, we ran into difficulties with this approach and eventually abandoned it due to the limited time available. For most of our studies the method we used to detect the presence of LPS-induced oligomerization of soluble TLR4/MD2 was to incubate the protein sample with LPS, crosslink the resulting complexes with glutaraldehyde, and then analyze the molecular weights using SDS-PAGE. This work is described in detail below.

An image of an SDS-PAGE analysis from R\&D (reduced and nonreduced) revealing sample quality is shown in Fig 4-1. The product is sold as a complex of TLR4 and MD2, but the SDS protocol breaks apart the complex into its component proteins. The single band at $92.5 \mathrm{kDa}$ is TLR4 and the two bands at 28 and 38 correspond to MD2. The calculated molecular weights for the nonglycosylated proteins are $70.6 \mathrm{kDa}$ for TLR4 and 19.2 for MD2. However, both proteins are glycosylated. MD2 is known to have two sites for glycoyslation. The majority of the sample is glycosylated at both sites and has a molecular weight of $35 \mathrm{kDa}$ while the remaining protein (lowest band in lanes 1 and 3 in Fig 4-1, at $27 \mathrm{kDa}$ ) appears to have only a single site glycosylated. The absence of bands above $92.5 \mathrm{kDa}$ indicates that either no oligomers of TLR4/MD2 are present in the sample or any oligomers that may have been present in the sample did not persist in the conditions of this SDS-PAGE protocol. If disulfide-linked dimers of TLR4/MD2 had been present, a higher molecular weight band would have appeared for the nonreducing conditions in lane 1. 


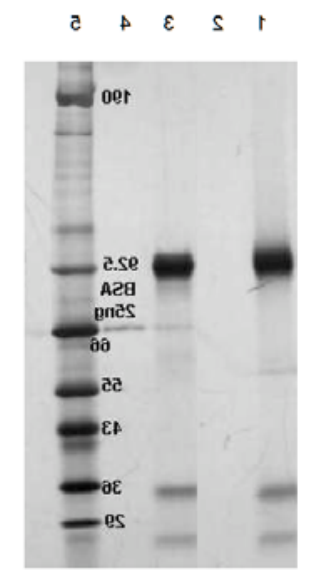

Figure 4-1. SDS PAGE of TLR4/MD2 samples from R\&D: Lane 1 nonreducing, lane 2 reducing.

Initially we tried to study LPS-induced dimerization with native gels using the sample of TL4/MD2 from R\&D, following a study reported by Park et al. (Cell paper and Nature paper). However, we were never successful, probably due to the need to determine the precise conditions of the gel preparation. This led us to follow the approach involving glutaraldehyde crosslinking. The latter approach is more intrusive, and requires controls to demonstrate that crosslinking does not occur of noncomplexed proteins, but is easier to perform.

In initial studies involving glutaraldehyde crosslinking we observed no effect of LPS Ra mutant on TLR4/MD2 (R\&D sample) in $40 \mathrm{mM}$ PBS buffer over a range of concentration from $0.04 \mathrm{uM}$ to $8 \mathrm{uM}$, covering a range of $\mathrm{pH}$ from 7.4 to 4.75, for $\mathrm{NaCl}$ concentration from $100 \mathrm{mM}-200$ $\mathrm{mM}$, and with CD14 and LBP present. Silver stained gels are shown in Fig 4-2. A control is shown in lane 3 in which no LPS or glutaraldehyde was added, but with 20 mM DTT (a reducing agent). This control is essentially the same as reported by R\&D (lane 3 in Fig 4-1) and a similar result was obtained. In lanes 4-11, two bands appear at about $90 \mathrm{kDa}$ and $120 \mathrm{kDa}$. This was consistently observed for glutadaldehyde crosslinked TLR4/MD2 when oligomerization did not occur. The two bands apparently correspond to crosslinked TLR4/MD2 complexes, with the difference in molecular weight resulting from MD2 having either a single site glycosylated or both sites glycosylated. The silver stained gel shows that some TLR4/MD2 oligomers are present after crosslinking even in the absence of LPS. This could be an artifact of glutaraldehyde crosslinking but may also indicate that a small amount of oligomeric TLR4/MD2 was present in the sample as received from $R \& D$. The main finding from this study was the lack of change upon addition of LPS shown in the comparisons of lanes 4 and 5, 6 and 7, and 8 and 9. 

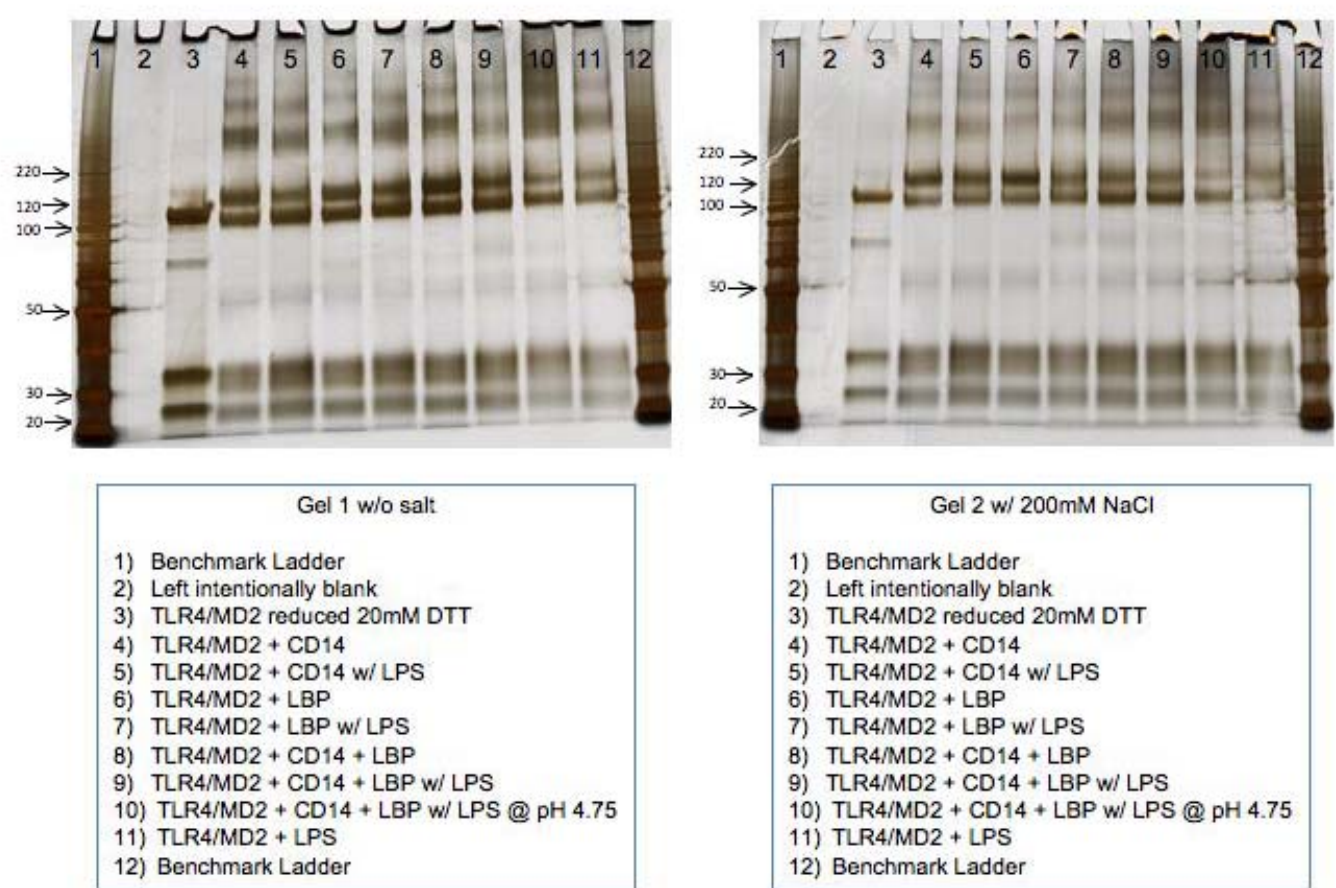

Gel 2 w/ $200 \mathrm{mM} \mathrm{NaCl}$
1) Benchmark Ladder
2) Left intentionally blank
3) TLR4/MD2 reduced $20 \mathrm{mM}$ DTT
4) TLR4/MD2 + CD14
5) TLR4/MD2 + CD14 w/ LPS
6) TLR4/MD2 + LBP
7) TLR4/MD2 + LBP w/ LPS
8) TLR4/MD2 + CD14 + LBP
9) TLR4/MD2 + CD14 + LBP w/ LPS
10) TLR4/MD2 + CD14 + LBP w/ LPS @ pH 4.75
11) TLR4/MD2 + LPS
12) Benchmark Ladder

Figure 4-2.

We also made a trial with TLR4 purchased from Abnova, and added purified MD2 from R\&D. The results are shown in Fig 4-3. A band consistently appears between $50 \mathrm{kDa}$ and $60 \mathrm{kDa}$ that we interpret as due to dimers of MD2. R\&D sells purified MD2 as a disulfide-linked dimer. We explored the importance of the order in which the various proteins (including CD14 and LBP) and LPS were combined. In all cases very little complexation of TLR4 with MD2 was observed. This is very likely due to the fact that MD2 was in dimer form and apparently does not complex with TLR4 as a dimer. Consequently, no LPS-induced dimerization was observed. The use of Abnova TLR4 with R\&D MD2 was dropped. 

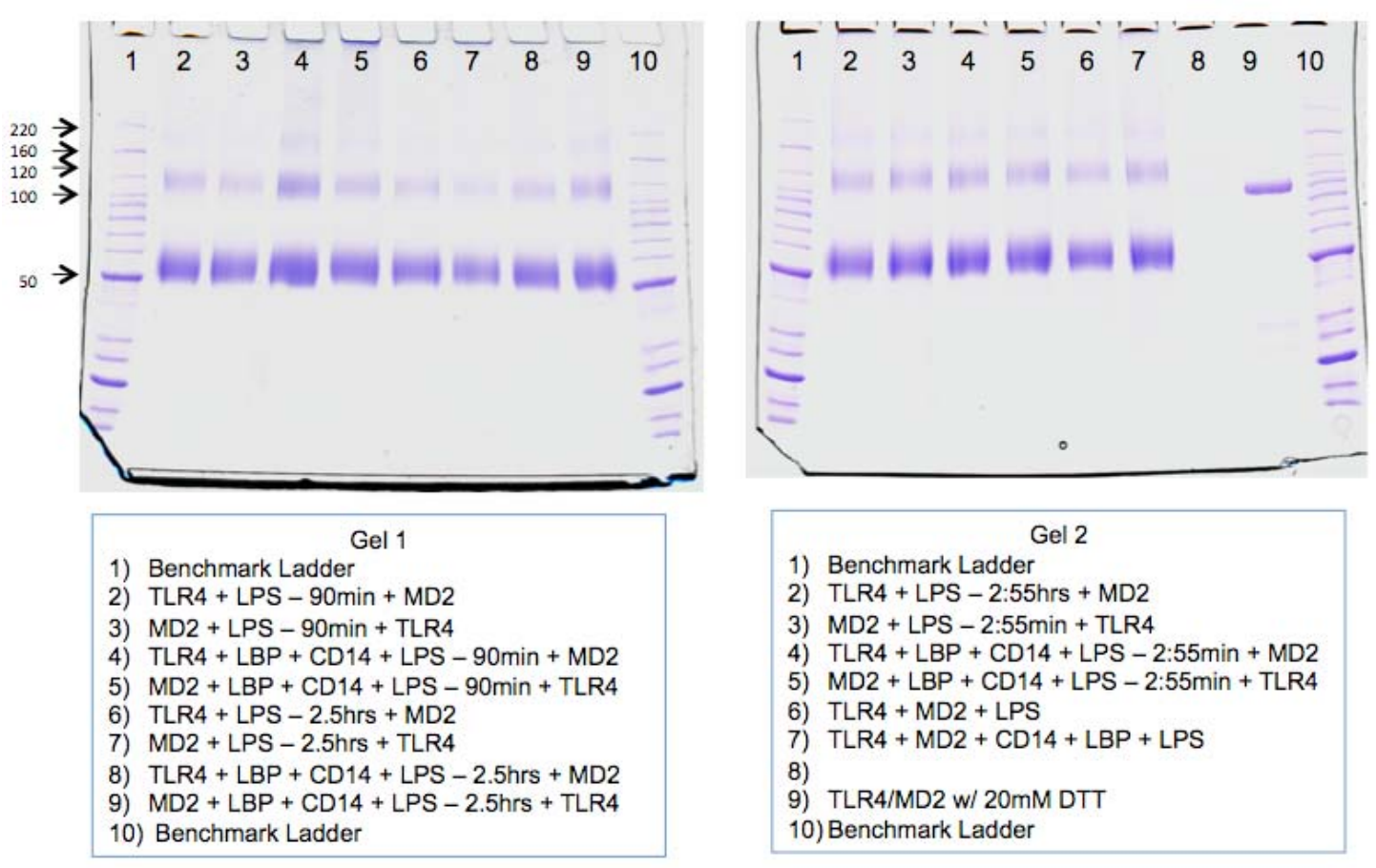

Figure 4-3. Abnova TLR4 combined with R\&D MD2. The band at $55 \mathrm{kDa}$ indicates the presence of dimers of MD2. No TLR4/MD2 complex formation was observed.

Subsequently, solid evidence for LPS oligomerization was observed in $20 \mathrm{mM}$ Tris $\mathrm{pH} 8.0$ with $200 \mathrm{mM} \mathrm{NaCl}$ (same buffer as used in the crystal structure studies) as shown in Fig 4-4. Controls are shown in lanes 3 and 4. This gel image shows results for $1.0 \mathrm{mg} / \mathrm{ml}-0.2 \mathrm{mg} / \mathrm{ml}$ TLR4/MD2, and also for a range of LPS molar concentration relative to TLR4/MD2 of 1x, 5x, and 10x. For both 5x and 10x LPS, strong oligomerization of TLR4/MD2 was observed with very little difference between them (lanes 5 and 6). This indicates that $5 x$ is a sufficient excess of LPS such that LPS is not limiting. On the other hand, LPS-induced oligomerization was much reduced when the molar concentration of LPS was the same as TLR4/MD2 (1x, lane 7). In our subsequent work, LPS was always present in excess by at least 10x relative to TLR4/MD2, and sobased on these data should not be a limiting factor. The concentration of TLR4/MD2 was varied from $1 \mathrm{mg} / \mathrm{ml}$ (lane 2) to $0.2 \mathrm{mg} / \mathrm{ml}$ (lanes 4-7). Both show significant amount of LPSinduced oligomerization. 

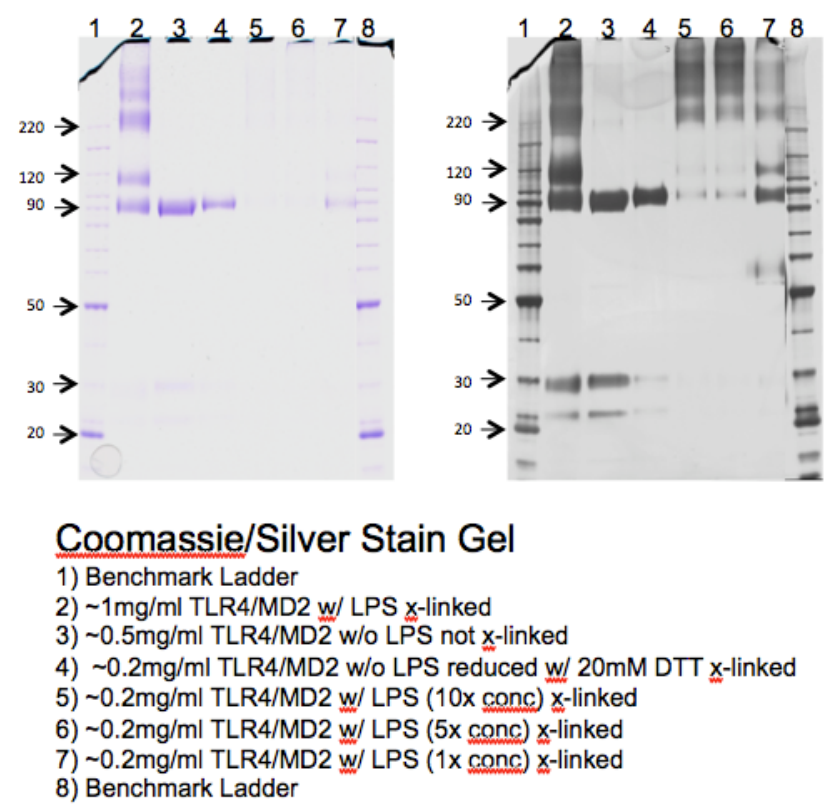

Figure 4-4. First observation of LPS-induced oligomerization of TLR4/MD2. Buffer was 20 mM Tris, pH 8.0 with $200 \mathrm{mM} \mathrm{NaCl}$.

Having a test for LPS-induced oligomerization we were able to resolve several questions important to our protocol and methods. These questions included the use of material that had been frozen in aliquots, the effect of dye labels on LPS-induced dimerization, and the range of concentration of LPS Ra mutant needed to induce complexation. During this time we used a stock of TLR4/MD2 from R\&D that had been in the freezer for greater than $1 \mathrm{yr}$. It was a substantial amount of protein that was purchased for use in the neutron reflectivity studies. We ran some tests with this material in the hope that it could still be used and could save on the purchase of new protein. In fact, LPS-induced oligomerization was observed with this sample as shown in Fig 4-5, lanes 2 - 4. However, TIRF imaging showed that this material contained substantial large protein aggregates, and so it was used only for qualitative studies. Labeling the protein had no effect on LPS-induced oligomerization that could be detected in the gel study. This is shown in Fig 4-5, lanes 3-4. We examined a range of LPS concentration from 0 uM to 50 uM (Fig 4-6, lanes 1-3). In most of the subsequent work we fixed the LPS concentration at 20 uM. We also determined the appropriate concentration range of glutaraldehyde for this study. At $0.5 \%$ glutaraldehyde no oligomerization was detected with SDS-PAGE (data not shown). Strong oligomerization was detected at $2 \%$ and also at 5\% (see Fig 4-6, Fig 4-7, and Fig 4-8). There is some concern that use of glutaraldehyde could crosslink some protein that had not formed a complex. This was tested by using glutaraldehyde at $0.5 \%$ to $5 \%$ but in absence of LPS Ra mutant (see Fig 4-8 below, lanes 1-4). No evidence for additional complex formation at higher glutaraldehyde concentration was observed. 


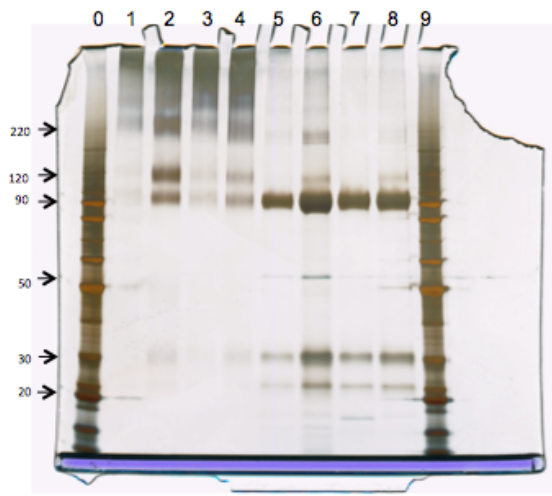

Silver Stain Gel

0) Benchmark Ladder

1) $\sim 0.2 \mathrm{mg} / \mathrm{ml}$ TLR $4 / \mathrm{MD} 2 \mathrm{w}$ LPS $x$-linked (sample never frozen)

2) $\sim 0.2 \mathrm{mg} / \mathrm{ml} \mathrm{TLR} 4 / \mathrm{MD} 2 \mathrm{w}$ LPS $x$-linked (from $2 \mathrm{yr}$ old stock that was frozen)

3) $\sim 0.2 \mathrm{mg} / \mathrm{ml}$ TLR4/MD2 Alexa488 $\mathrm{w}$ LPS $\mathrm{x}$-linked (from $2 \mathrm{yr}$ old stock that was frozen)

4) $\sim 0.2 \mathrm{mg} / \mathrm{ml}$ TLR4/MD2 Alexa532 $\mathrm{w}$ LPS $\mathrm{x}$-linked (from $2 \mathrm{yr}$ old stock that was frozen)

5) $\sim 0.2 \mathrm{mg} / \mathrm{ml}$ TLR4/MD2 w/o LPS, reduced (sample never frozen)

6) $\sim 0.2 \mathrm{mg} / \mathrm{ml}$ TLR4/MD2 w/o LPS, reduced (from 2 yr old stock that was frozen)

7) $\sim 0.2 \mathrm{mg} / \mathrm{ml}$ TLR4/MD2 Alexa 488 w/o LPS, reduced (from $2 \mathrm{yr}$ old stock that was frozen)

8) $\sim 0.2 \mathrm{mg} / \mathrm{ml}$ TLR4/MD2 Alexa532 w/o LPS, reduced (from $2 \mathrm{yr}$ old stock that was frozen)

Figure 4-5. SDS PAGE: 2 yr old TLR4 stock, effect of labeling.

Subsequent to this preliminary work, we ran several studies involving a range of concentration of TLR4/MD2 from $1.6 \mathrm{uM}$ to $0.08 \mathrm{uM}$ at fixed LPS concentration of $20 \mathrm{uM}$. This LPS concentration is in all cases greater than 10 times that of the TLR4/MD2, and so assumed to not be a limiting factor. The results consistently showed LPS-induced oligomerization was nearly nonexistent at $0.08 \mathrm{uM}$ and became apparent at $0.16 \mathrm{uM}$ and became steadily stronger with increasing TLR4/MD2 conc. Results are shown in Fig 4-6, Fig 4-7, and Fig 4-8. For the gel on the right had side in Fig 4-8 the loading of protein in each lane was held constant. We also performed a western blot, using a primary antibody to MD2 under similar conditions. The results are shown in Fig 4-9. The results in Fig 4-6 - Fig 4.9 show that the complexation occurs only when a relatively high concentration of receptor is present, and seems to explain why LPSinduced complexation of membrane-bound TLR4/MD2 was not observed in the earlier TIRF studies, as that method can only be performed at very low receptor densities. 

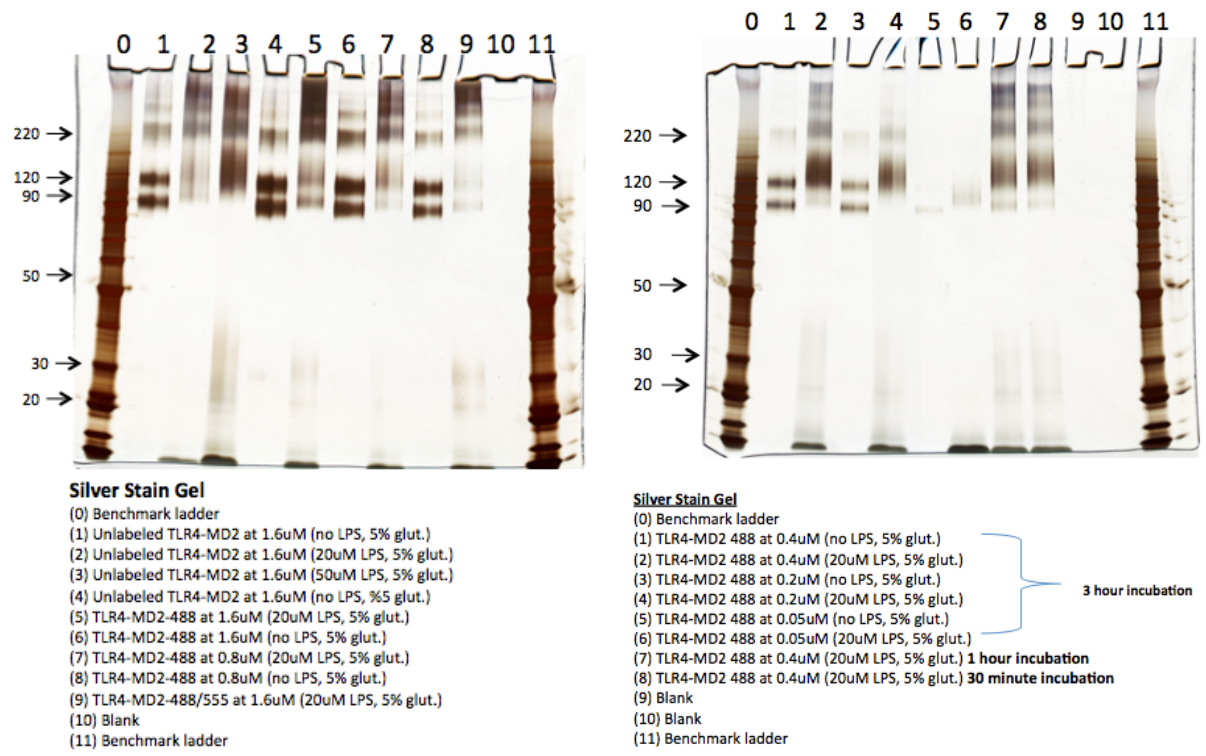

Figure 4-6. SDS PAGE: effect of TLR4 conc. at 20 uM LPS
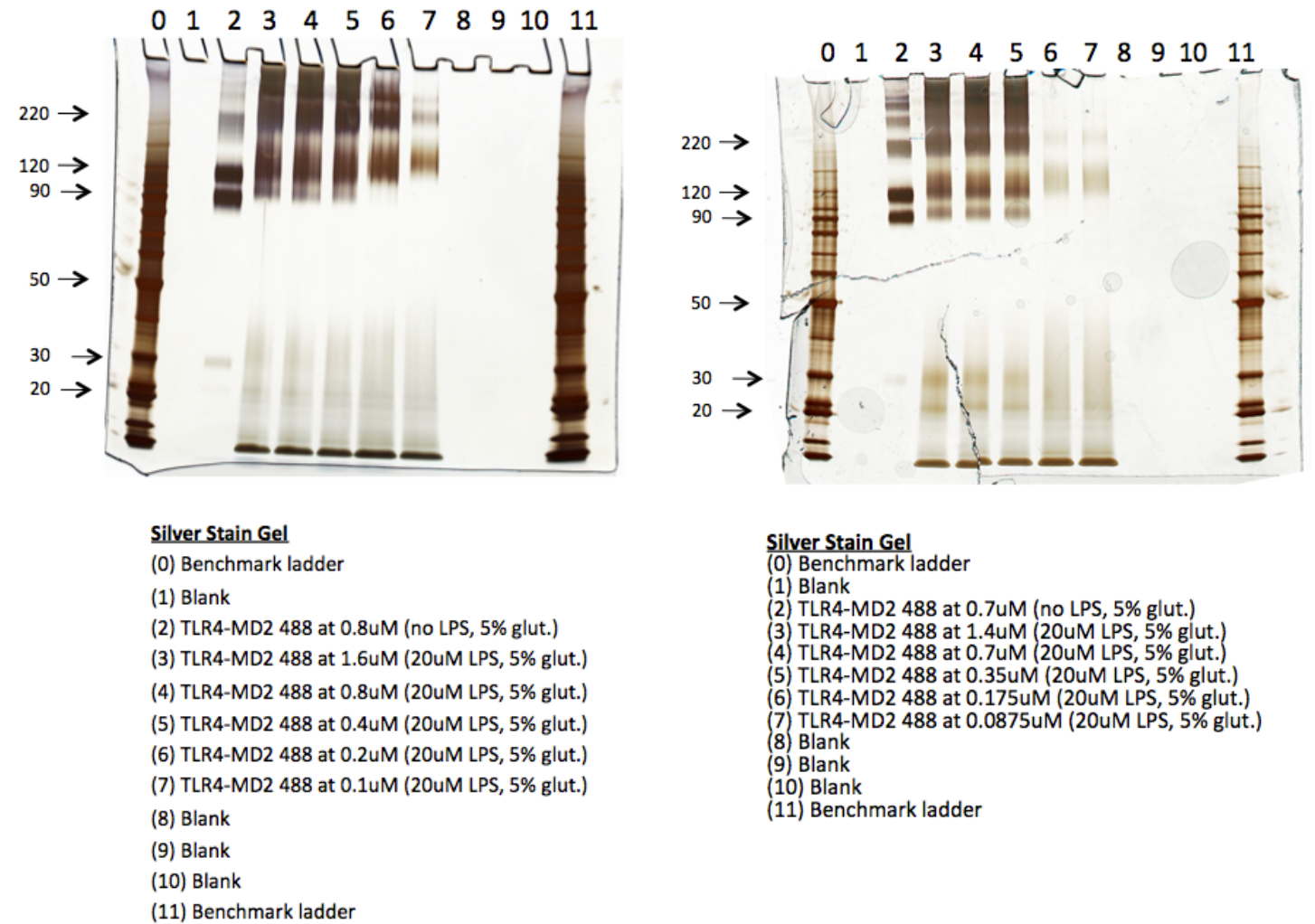

\section{Silver Stain Gel}

0) Benchmark ladder

(1) Blank

(2) TLR4-MD2 488 at $0.74 M$ (no LPS, $5 \%$ glut.)

(3) TLR4-MD2 488 at $1.4 \mathrm{uM}$ (20uM LPS, 5\% glut.)

(4) TLR4-MD2 488 at 0.7 uM (20uM LPS, $5 \%$ glut.)

(5) TLR4-MD2 488 at 0.35 uM (20uM LPS, 5\% glut.)

(6) TLR4-MD2 488 at 0.175 uM (20uM LPS, $5 \%$ glut.)

7) TLR4-MD2 488 at 0.0875 uM (20uM LPS, $5 \%$ glut.)

8) Blank

(9) Blank

(10) Blank

(11) Benchmark ladder

(11) Benchmark ladde

Figure 4-7. SDS PAGE: effect of TLR4 conc. at 20 uM LPS 


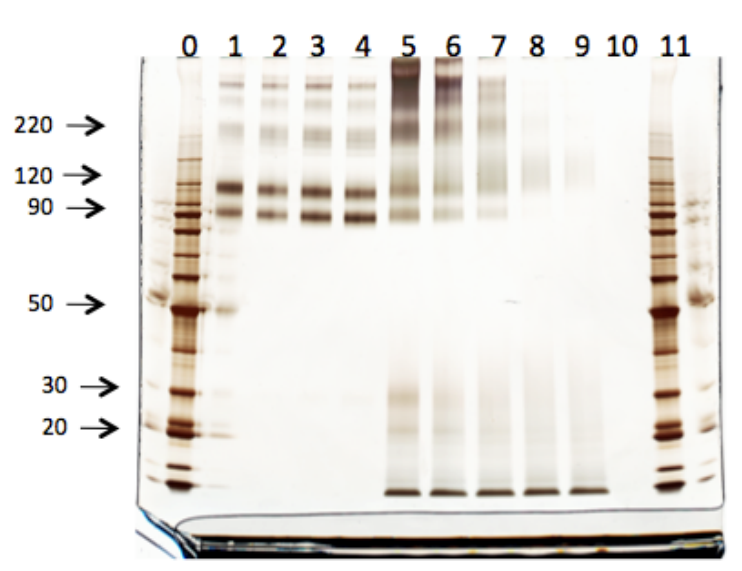

\section{Silver Stain Gel}

(0) Benchmark ladder

(1) TLR4-MD2 488 at 0.7 uM (no LPS $5 \%$ glut.)

(2) TLR4-MD2 488 at 0.7 uM (no LPS, $2 \%$ glut.)

(3) TLR4-MD2 488 at 0.7 uM (no LPS, $1 \%$ glut.)

(4) TLR4-MD2 488 at $0.7 \mathrm{uM}$ (no LPS, $0.5 \%$ glut.)

(5) TLR4-MD2 488 at 1.4 uM (20uM LPS, $2 \%$ glut.)

(6) TLR4-MD2 488 at 0.7 uM (20uM LPS, $2 \%$ glut.)

(7) TLR4-MD2 488 at 0.35 uM (20uM LPS, $2 \%$ glut.)

(8) TLR4-MD2 488 at $0.175 u$ M (20uM LPS, $2 \%$ glut.)

(9) TLR4-MD2 488 at 0.0875 uM (20uM LPS, $2 \%$ glut.)

(10) Blank

(11) Benchmark ladder

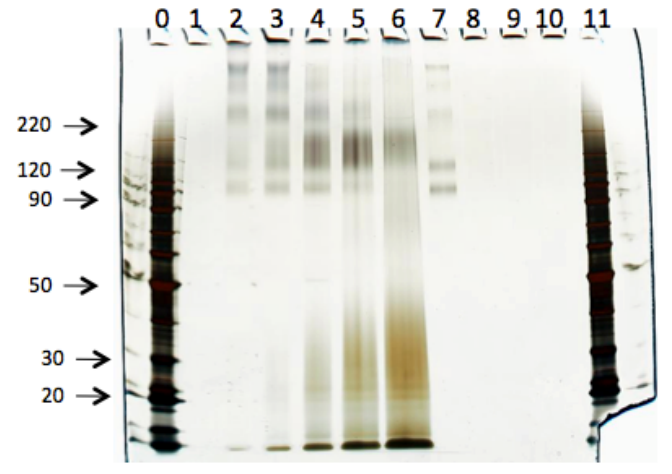

\section{Silver Stain Gel}

(0) Benchmark ladder

(1) Blank

(2) TLR4-MD2 488 at $1.4 \mathrm{UM}$ (20uM LPS, $2 \%$ glut.)

3) TLRA-MD2 488 at $0.74 \mathrm{MM}(20 \mathrm{um}$ LPS, $2 \%$ glut.)

(4) TLR4-MD2 488 at 0.35 uM (20um LPS, $2 \%$ glut.)

(5) TLR4-MD2 488 at 0.175 uM (20uM LPS, $2 \%$ glut.)

(6) TLR4-MD2 488 at 0.0875 uM (20uM LPS, $2 \%$ glut.)

(7) TLR4-MD2 488 at $0.7 \mathrm{uM}$ (no LPS, $2 \%$ glut.)

(8) Blank

(9) Blank

10) Blank

(11) Benchmark ladder

Figure 4-8. SDS PAGE: effect of TLR4 conc. at 20 uM LPS, also control study of glut. conc in absence of LPS. The amount of protein in each lane was constant for the gel on the right side.

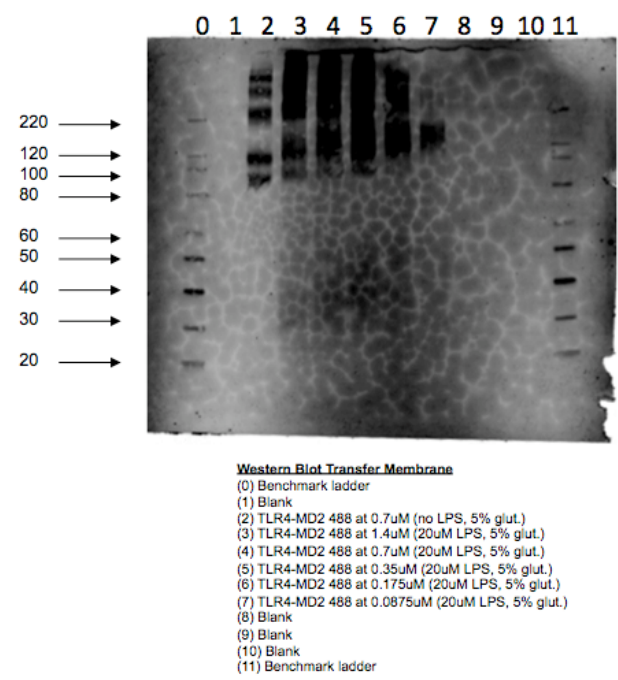

Figure 4-9. Western blot using primary antibody to MD2 showing LPS-induced oligomerization of TLR4/MD2. 
Having established the concentration range over which LPS-induced complexation of soluble TLR4/MD2 occurs, we examined the effect of partially deglycosylating TLR4/MD2 and also the effect of the presence of CD14 and LBP. The motivation for studying the effect of deglycosylation was possibility that a better FRET signal upon complexation would result, and also the possibility that deglycosylation would lead to complexation at lower concentrations. We followed the protocol reported by the Kim et al (Cell 2007) and Park et al (Nature 2009), but we were unable to reproduce their result of complete deglycosylation. Our best results for several trials are shown in Fig 4-10, which correspond to a molar ratio of TLR4 to PNGase F ranging from 5.8:1 to 1.2:1. Park et al reported using a ratio of 3:1. Due to the lack of time remaining, we nevertheless performed a study of LPS-induced oligomerization with this sample. The results (not shown) indicate that partial deglycosylation had no apparent effect on LPS-induced oligomerization. The partially deglycosylated TLR4/MD2 was also used in a FRET study described in section 4.1.3.
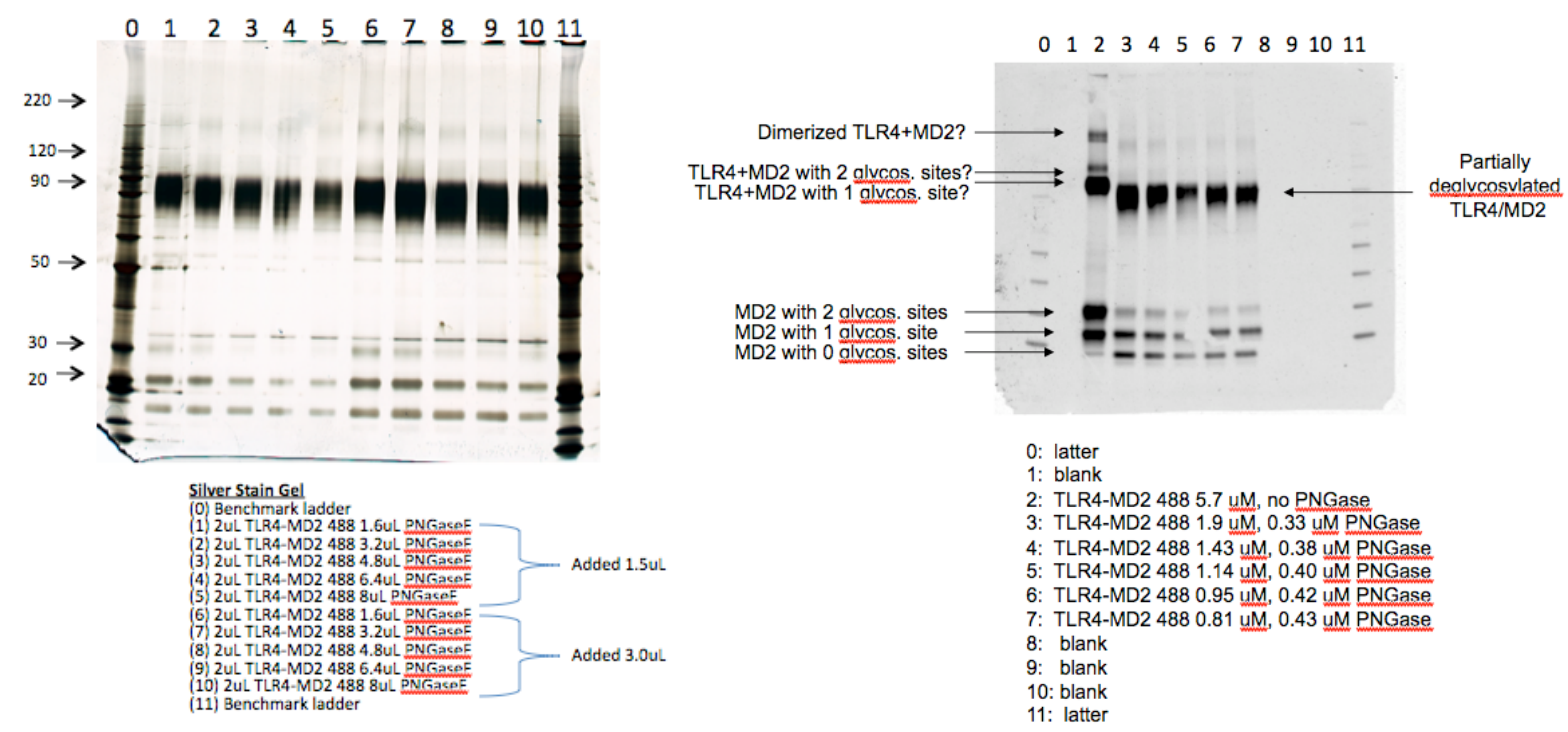

Figure 4-10. Attempt at deglycosylating the TLR4/MD2 sample: SDS PAGE (left), western blot using primary antibody to MD2 (right).

Regarding the effects of CD14 and LBP, we examined the effect of each protein alone on the LPS-induced oligomerization of TLR4. Those results are shown in Fig 4-11. While CD14 at 0.5 $\mathrm{uM}$ had no detectable effect on LPS-induced oligomerization, the presence of LBP at $0.5 \mathrm{uM}$ greatly increased the affinity such that strong oligomerization was observed for a TLR4/MD2 concentration of $0.08 \mathrm{uM}$. The presence of both proteins, $0.5 \mathrm{uM} \mathrm{CD14}$ and $0.5 \mathrm{uM} \mathrm{LBP}$, also a very significant effect as shown in Fig 12. In that case strong LPS-induced oligomerization was present at $0.01 \mathrm{uM}$ TLR4/MD2, whereas none was present at that concentration in the absence of CD14 and LBP or with CD14 alone. It is likely that this effect is due to LBP extracting LPS from micelles and shuttling it to TLR4/MD2. However, since LBP alone has a strong effect it is not clear what role CD14 plays. The fact that CD14 has a critical effect on TLR4 signaling has been well documented in the literature (Godowski, Nature Immunol. 2005; Jiang, Nat. Immunol. 2005). More work is needed to understand this. Also, since LBP facilitates oligomerization at 
lower receptor densities, it would be important to repeat the TIRF studies in the presence of CD14 and LBP.

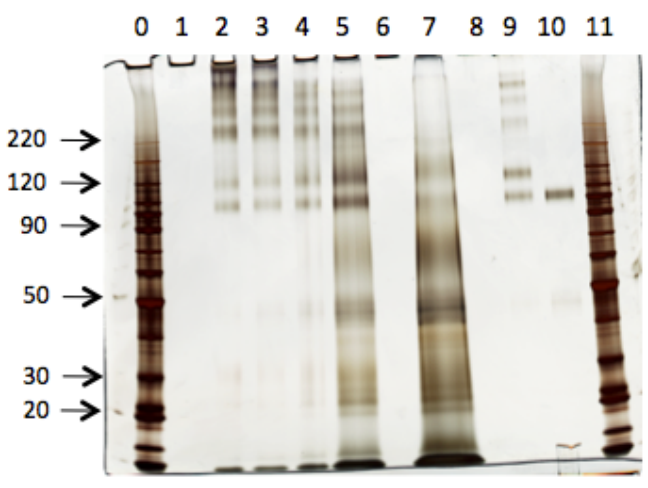

Silver Stain Gel

(O) Benchmark ladder

(1) Blank

(2) TLR4-MD2 at $1.4 \mathrm{uM}$ (20 uM LPS, 0.5 uM CD14, $2 \%$ glut.)

(3) TLR4-MD2 at 0.7 UM (20 uM LPS, 0.5 uM CD14, $2 \%$ glut.)

(4) TLR4-MD2 at $0.35 \mathrm{uM}$ (20 uM LPS, 0.5 uM CD14, $2 \%$ glut.)

(5) TLR4-MD2 at 0.175 UM (20 uM LPS, 0.5 uM CD14, $2 \%$ glut.)

(6) Blank

7) TLR4-MD2 at $0.08 u M(20 u M$ LPS, $0.5 u M$ CD14, $2 \%$ glut.)

(8) Blank

(9) TLR4-MD2 at $0.7 \mathrm{uM}$ (NO uM LPS, 0.5 uM CD14, $2 \%$ glut.)

(10) TLR4-MD2 at 0.7uM (NO uM LPS, $0.5 \mathrm{uM} \mathrm{CD14,} \mathrm{NO} \mathrm{glut.)}$

(11) Benchmark ladder

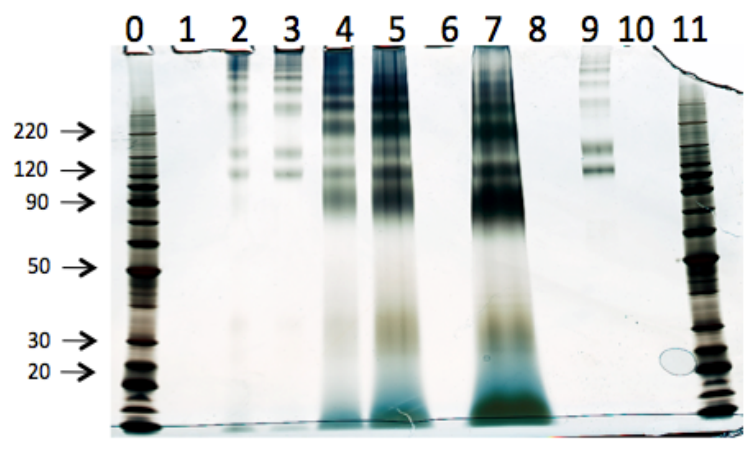

Silver Stain Gel

(0) Benchmark ladder

(1) Blank

(2) TLR4-MD2 at 1.4uM (20 uM LPS, 0.5 uM LBP, $2 \%$ glut.

(3) TLR4-MD2 at $0.7 \mathrm{MM}(20 \mathrm{UM}$ LPS, $0.5 \mathrm{UM}$ LBP, $2 \%$ glut.)

(4) TLR4-MD2 at $0.35 \mathrm{uM}$ (20 ॥M LPS, 0.5 ॥M LBP, $2 \%$ glut.)

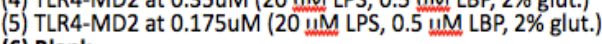

(6) Blank

(7) TLR4-MD2 at $0.08 u M$ ( 20 „M LPS, 0.5 ॥M LBP, $2 \%$ glut.)

(8) Blank

(9) TLR4-MD2 at 0.7 UM (NO LPS, 0.5 แM LBP, $2 \%$ glut.) CONTROL

(10) Blank

(11) Benchmark ladder

Figure 4-11. Effect of CD14 (left) and LBP (right) on LPS-induced oligomerization of TLR4/MD2.

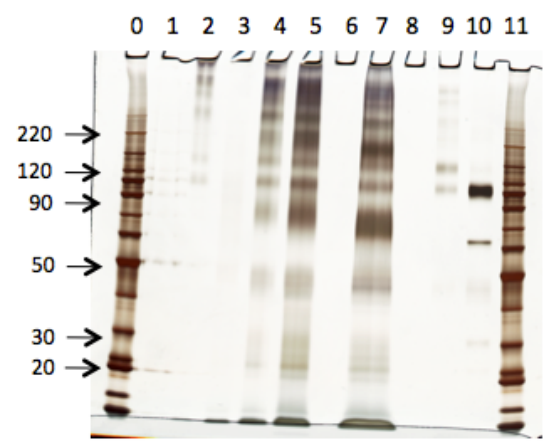

Silver Stain Gel

(0) Benchmark ladder

(1) Blank

(2) TLR4-MD2 at 1.4uM (20 uM LPS, 0.5 uM CD14, 0.5 UM LBP, $2 \%$ glut.)

(3) TLR4-MD2 at $0.74 \mathrm{M}$ (20 UM LPS, $0.5 \mathrm{UM}$ CD14, $0.5 \mathrm{UM}$ LBP, $2 \%$ glut.)

(4) TLR4-MD2 at 0.35 uM (20 uM LPS, 0.5 uM CD14, 0.5 uM LBP, $2 \%$ glut.)

(5) TLR4-MD2 at 0.175 UM (20 џM LPS, 0.5 uM CD14, 0.5 uM LBP, $2 \%$ glut.)

(6) Blank

(7) TLR4-MD2 at $0.08 \mathrm{uM}$ (20 ЩM LPS, 0.5 uM CD14, 0.5 uM LBP, $2 \%$ glut.)

(8) Blank

(9) TLR4-MD2 at $0.7 \mathrm{UM}$ (NO uM LPS, 0.5 uM CD14, 0.5 uM LBP, $2 \%$ glut.)

(10) TLR4-MD2 at $0.7 \mathrm{MM}$ (NO uM LPS, $0.5 \mathrm{uM}$ CD14, NO glut.)

(11) Benchmark ladder

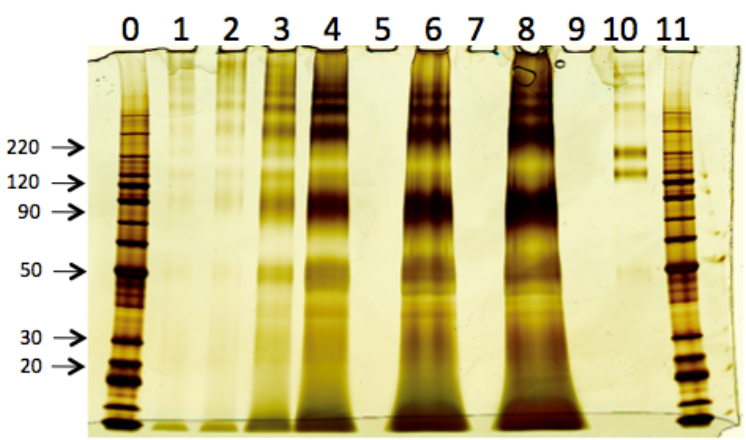

Silver Stain Gel

(1) Benchmark ladder

(2) TLR4-MD2 at $0.175 \mathrm{uM}$ (20 uM LPS, 0.5 uM CD14, 0.5 UM LBP, $2 \%$ glut.)

(3) TLR4-MD2 at $0.08 \mathrm{uM}$ ( 20 uM LPS, 0.5 uM CD14, 0.5 uM LBP, $2 \%$ glut.)

(4) TLR4-MD2 at 0.04uM (20 uM LPS, 0.5 uM CD14, 0.5 uM LBP, $2 \%$ glut.

(5) Blank

(6) TLR4-MD2 at 0.02uM (20 uM LPS, 0.5 uM CD14, 0.5 uM LBP, $2 \%$ glut.)

(7) Blank

(8) TLR4-MD2 at 0.01uM (20 uM LPS, 0.5 uM CD14, 0.5 uM LBP, $2 \%$ glut.)

(9) Blank

(10) TLR4-MD2 at $0.7 \mathrm{MM}$ (NO LPS, 0.5 ॥M CD14, 0.5 ॥M LBP, $2 \%$ glut.) CONTROL

(11) Benchmark ladder

Figure 4-12. Effect of CD14 and LBP. a) Concentration range of TLR4/MD2 extends to down to $0.08 \mathrm{uM}$. b) Concentration range of TLR4/MD2 extends to down to $0.01 \mathrm{uM}$. Strong oligomerization of TLR4 is present at $0.01 \mathrm{uM}$ with LBP and CD14 present. 


\subsubsection{Crosslinking/TIRF}

In an attempt to quantify the amount of each type of oligomer (dimer, trimer, tetramer, etc) present we exposed a portion of the sample (same as used for the gel studies) to a bilayer of $10 \%$ DOIDA/ $\mathrm{Cu}^{2+}+90 \%$ POPC supported on a glass cover slip incorporated into a TIRF liquid cell. Then we took TIRF images and analyzed the distribution of intensities for the observable spots. An sample image is given in Figure 4-13 and intensity distributions for a series of samples is given in Figure 4-14. For this work we used TLR4/MD2 labeled with Alexa488 at roughly 1 dye/protein. For a control, we first used a sample of the protein in absence of LPS. In that case a fairly narrow distribution resulted as shown in Fig 4-14. For a sample exposed to LPS Ra mutant (corresponding to the gel image on the right hand side in Fig 4-7), the distribution of intensities is much broader and includes much higher values due to the presence of TLR4/MD2 oligomers. However, quantification of the numbers of each type of oligomer was complicated by the fact that the intensities did not fall into discrete bands as expected. Rather, there was a continuous distribution of intensities as shown in Fig 4-14. We can conclude that a range of oligomers up to pentamers are present, but we have not yet found a way to quantify the number of each type of oligomer present.
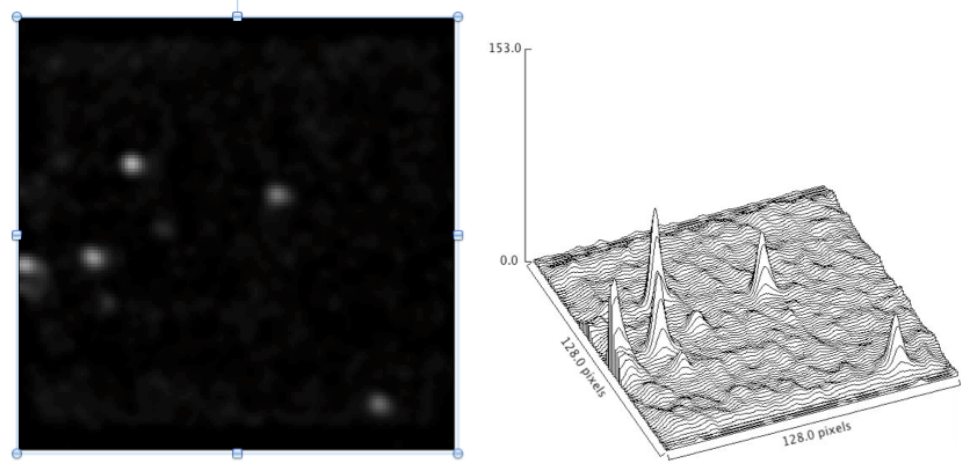

Figure 4-13. Sample TIRF image and intensity map (1.4 uM TLR4-MD220 uM LPS Ra mut).
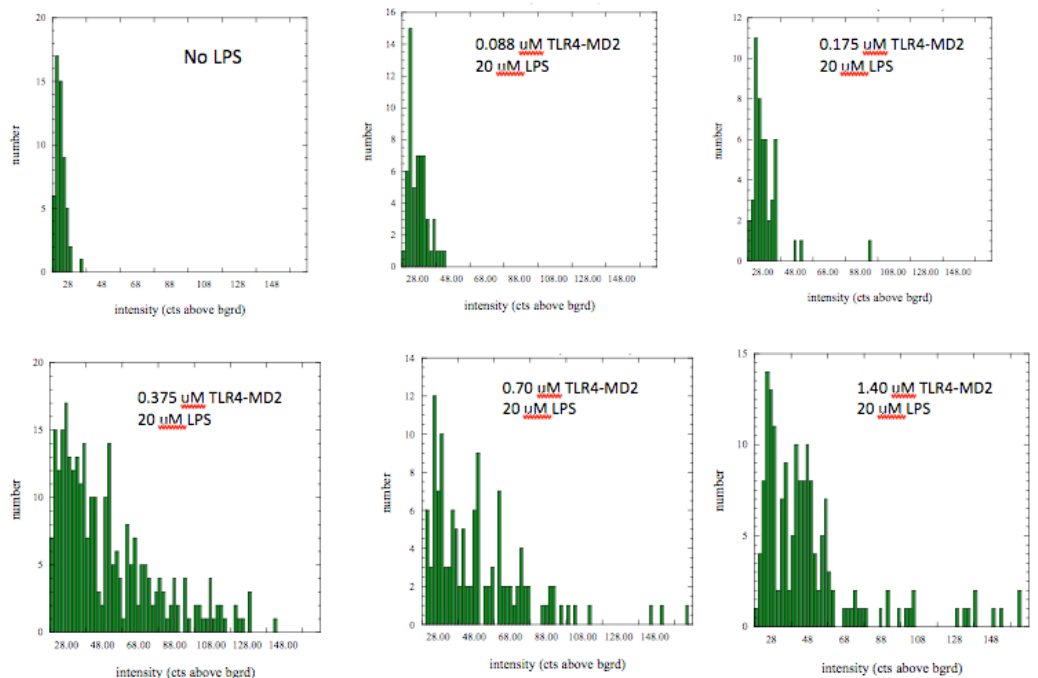

Figure 4-14. TIRF intensity distributions for samples corresponding to the gel image on the right side in Fig 4-7. 


\subsubsection{FRET}

We tried use FRET to detect the assembly of the TLR4-MD2 complex in solution. In our trials we used the FRET pair Alexa 488 and Alex 555 with Forster radius of $70 \AA$. Measurements were made with a Nanodrop3000. Sampel spectra from the two dyes are shown in Fig 4-15. As a control, we first conjugated the two dyes to the same TLR4/MD2 sample. The number of each type of dye molecule per TLR4/MD2 was 1.5 (Alexa 555) and 1.5 (Alexa 488). In that case upon excitation of the 488 dye, a strong FRET signal was detected, as shown in Fig 4-16 (left panel). Then two samples of TLR4/MD2 were labeled separately with Alexa 488 and Alexa 555. The samples were combined (3.3 uM of TLR4/MD2 488 and $3.3 \mathrm{uM}$ of TLR4/MD2 555) and incubated for $30 \mathrm{~min}$ at room T with $30 \mathrm{uM}$ LPS Ra mutant. In this case the number of dye molecules per TLR4/MD2 was 2.2 (Alexa 555) and 1.45 (Alexa 488). The results, shown in Fig 4-16 (middle panel), indicate only a weak FRET signal. A portion of the sample was crosslinked and analyzed by SDS-PAGE, however inadvertently only $0.5 \%$ glutaraldehyde was used which is too low to observe crosslinked complexes. However a subsequent run was made using $0.8 \mathrm{uM}$ TLR4/MD2 488 and 0.8 uM TLR4/MD2 555 (see lane 9 of Fig 4-6 right side) which showed clear evidence of LPS-induced oligomerization. Thus, despite the strong evidence for LPSinduced oligomerization in solution, we observed very little FRET signal.
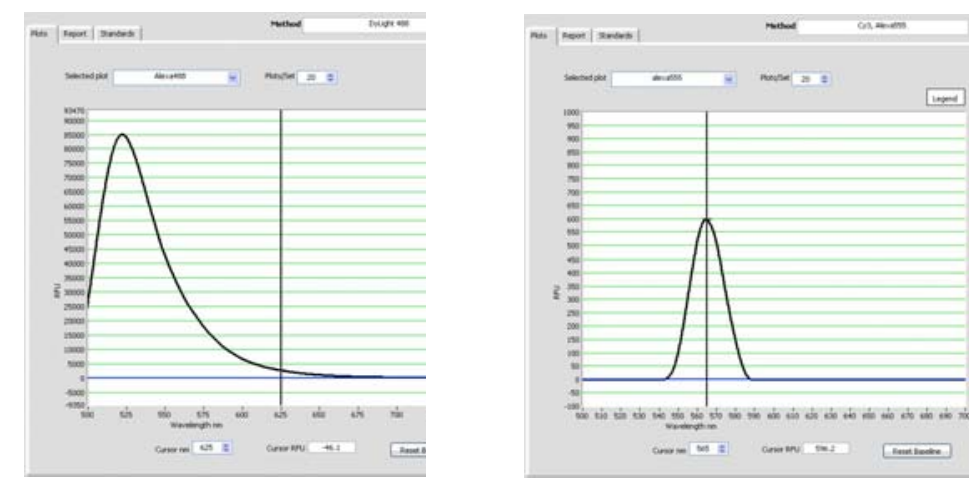

Figure 4-15. Spectra for Alexa 488 (left) and Alexa 555 (right)
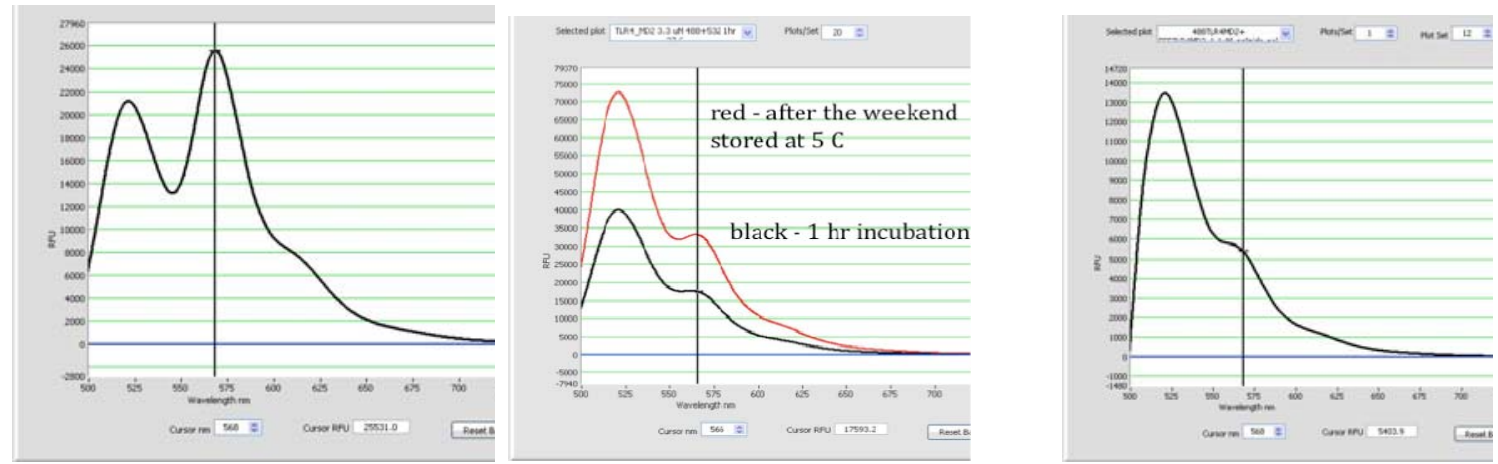

Figure 4-16. Results of FRET study. (left panel) TLR4/MD2 labeled with Alexa 488 and Alexa 555. (center panel) $3.3 \mathrm{uM}$ TLR4/MD2-488 mixed with $3.3 \mathrm{uM}$ TLR4/MD2-555 and incubated with $30 \mathrm{uM}$ LPS Ra mutant. (right panel) ) $1.1 \mathrm{uM}$ TLR4/MD2-488 mixed with $1.1 \mathrm{uM}$ TLR4/MD2-555 but no LPS (control). 
Finally, we repeated this with partially deglycosylated TLR4/MD2. The results (not shown) are similar to those in Fig 4-16 and showed very little FRET signal.

A possible explanation for the weakness of the FRET signal is that the dye molecules on average are too far apart for the TLR4/MD2 oligomeric complexes (Fig 4-16 center panel), whereas for the TLR4/MD2 monomer the two dyes are sufficiently close for strong energy transfer (Fig 4-16 left panel). The dimensions of monomeric and dimeric TLR4/MD2 from recently published crystal structures are shown in Figs 4-17. In this work the dyes conjugated to amine groups on the proteins. The distribution of lysines on TLR4/MD2 are shown in the right panel in Fig 4-17. Given the random distribution of lysines, it is possible that with the dimeric TLR4/MD2 and only 1-2 dye of each type per protein the distance between 488 and 555 in most cases is too large for efficient FRET. To ensure that the dyes are within the Forster radius when the complex assembles, the best approach is to genetically alter the protein to place the dyes in the most strategic locations. However, because expression of TLR4/MD2 was unsuccessful, we were not able to do that.
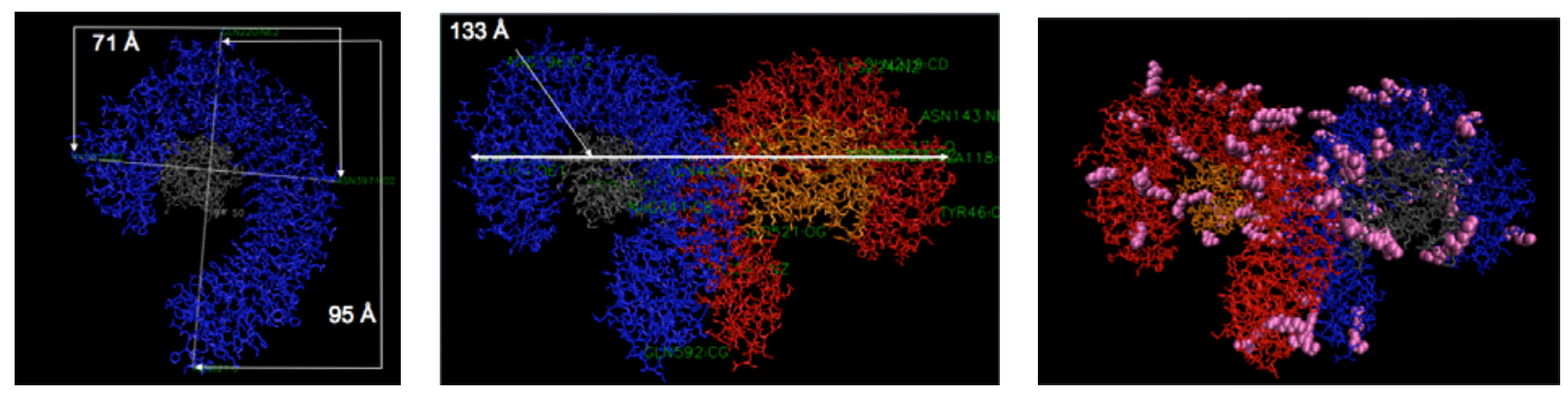

Figure 4-17. Dimension of monomeric and dimeric TLR4/MD2 from recently published crystal structures (Kim et al Nature 2009). The distribution of lysines are shown in the panel on the right.

\subsubsection{CryoEM}

Initial experiments with lipid IVa mixed with TLR4/MD2 indicated strong aggregation of the complex upon LPS binding. Further study was needed to find optimal conditions for LPS binding to the complex. This was not pursued due to time constraints. Rather, the cryoEM effort focused on TLR4/MD2 bound to liposomes, discussed further below. 


\subsection{Membrane-bound TLR4/MD2}

\subsubsection{Synthesis of DOIDA}

DOIDA, a fluid phase lipid with a metal-chelating IDA headgroup, was synthesized following a previously published method.(Pack) This was an important step as the use of DOIDA avoids aggregation or domain formation that occurs with the gel-phase lipid DSIDA in the absence of protein or ligand. Such domain formation precludes the study of single isolated membranebound TLR4/MD2 monomers. With DOIDA, isolated membrane-bound TLR4/MD2 monomers can be examined before and after introducing ligand. This was a critical step in developing diffusional and cross correlation studies of ligand-induced dimerization of TLR4/MD2.

\subsubsection{Results: FCS}

Fluorescence correlation methods were used both to characterize the lipid membrane systems we developed for binding TLR4 and to search for formation of TLR4 containing complexes under a variety of conditions.

\section{Membrane Characterization}

The first protein binding membrane we developed made use of DSIDA in a matrix of POPC. The IDA headgroup on the lipid was loaded with $\mathrm{Cu}^{+2}$ which binds proteins to the membrane through His tags on the protein. Membranes containing 10\% DSIDA were formed on glass substrates using vesicle fusion. Upon addition of $\mathrm{Cu}^{+2}$ these membranes were found to undergo phase separation to form domains rich in DSIDA. These domains bind proteins with very high affinity. Diffusion in these membranes was probed using FCS both in the domains and in the matrix POPC using the lipid probe Bodipy 530/550 HPC. Fluorescence correlation was performed with a custom confocal microscope using excitation at $532 \mathrm{~nm}$ and a custom data acquisition system that records time stamped arrivals of each detected photon. Correlations were performed offline with a software correlation routine. Figure 4-18 shows the fluorescence autocorrelations determined for excitation in a domain and in the matrix lipid. 


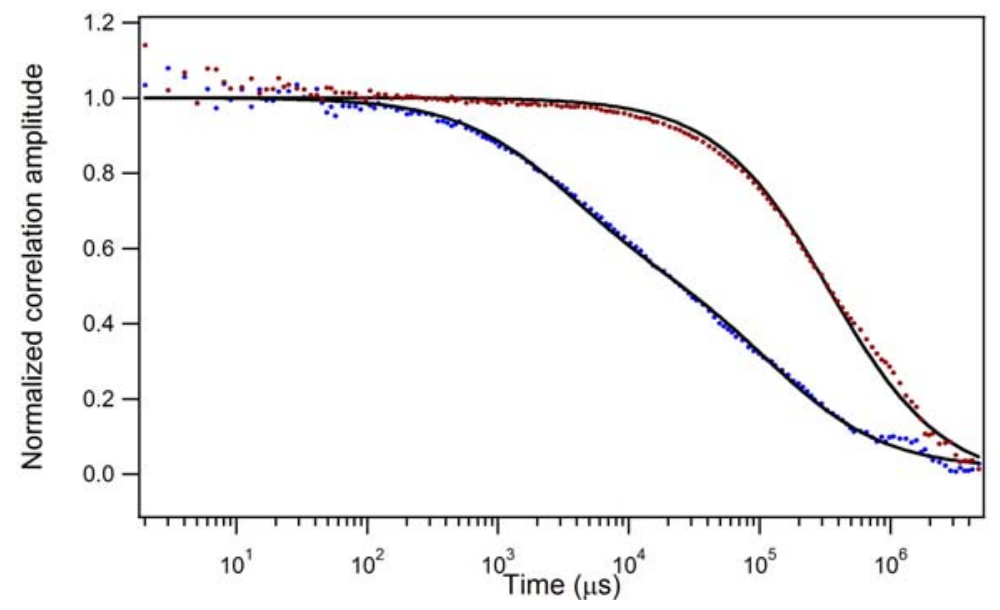

Figure 4-18. Fluorescence correlation spectroscopy of a POPC region (blue dots) and a DSIDA region (red dots) of a 10\% DSIDA/POPC membrane following $\mathrm{CuCl}_{2}$ treatment. Fits to the data used to determine diffusion coefficients are shown as black lines. The data were fit to standard correlation function forms for two dimensions.

The very slow diffusion seen in the DSIDA region indicates the formation of gel phase domains. While these domains have very high affinity for proteins a more fluid membrane with faster diffusion is advantageous for our studies of the interaction of TLR4 monomers to form complexes. For this reason we developed another membrane system using DOIDA in POPC. Membranes formed from a mixture of 10\% DOIDA in POPC on glass substrates were found to be mostly homogeneous (Fig. 4-19) and fluid even after the addition of $\mathrm{Cu}^{+2}$ to enable protein binding.

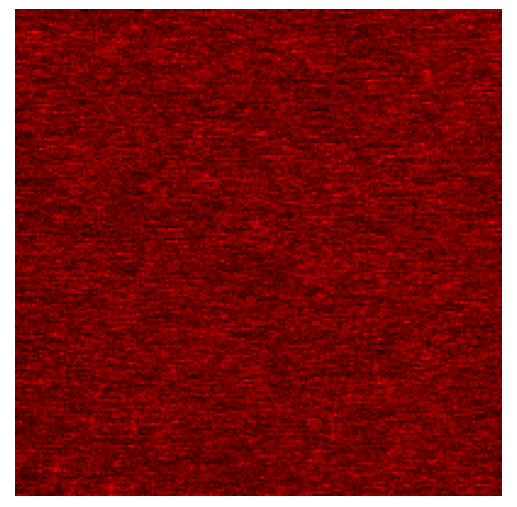

Figure 4-19. Confocal image of a $\mathrm{Cu}^{+2}$-DOIDA/POPC membrane shows binding of proteins in a fluid phase. The protein coverage is quite uniform and the proteins are mobile.

FCS measurements of TLR4/MD2 labeled with Atto 532 dye bound to a DOIDA/POPC membrane are shown in Figure 4-20. The autocorrelation curve shows the diffusion on this membrane is consistent with a fluid phase. 


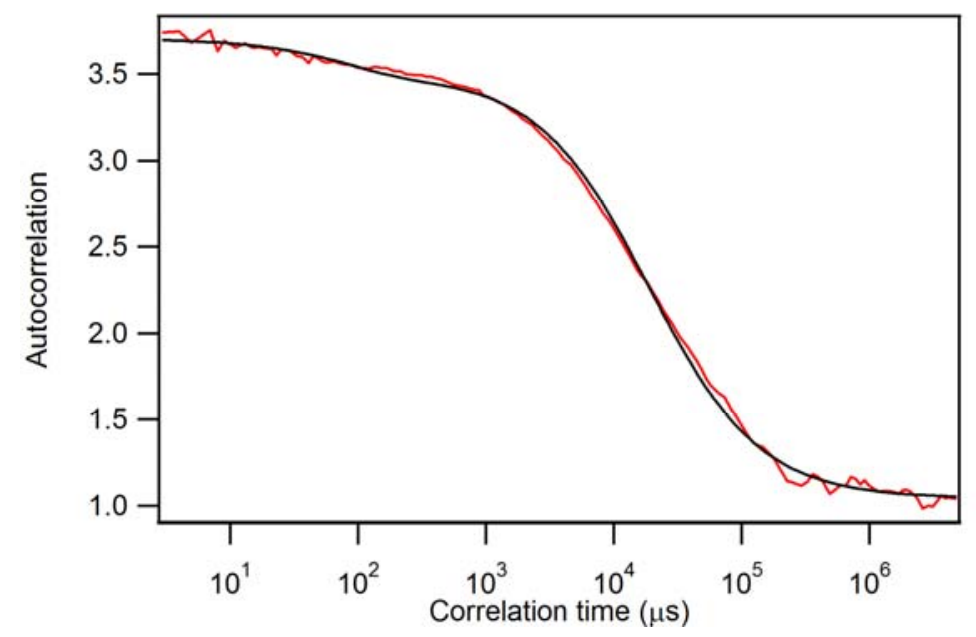

Figure 4-20. FCS measurement of Atto 532 labeled TLR4/MD2 bound to a DOIDA/POPC membrane. The black fit has a single diffusion coefficient, expected for a fluid membrane.

Experiments to detect complex formation of TLR4/MD2 due to Lipid A or LPS addition were performed with this membrane system. FCS measurements of the bound TLR4/MD2 on DOIDA/POPC membranes were taken before and after Lipid A addition. While small changes in the correlation curves were observed, they were found to be comparable to variations observed looking at different points on the membrane. To make measurements more sensitive to complex formation rather than absolute diffusion coefficients we began fluorescence cross correlation experiments and FRET experiments described below.

\section{Fluorescence cross correlation to detect complex formation}

We implemented fluorescence cross correlation experiments by labeling two samples of TLR4/MD2 with different dyes, Alexa Fluor 514 and Atto 590. Cross correlation of the time histories of the fluorescence from the two different dyes is used to determine whether fluctuations in their fluorescence intensity are related. This correlation is sensitive to the extent of aggregation between the two labeled protein populations. The two dyes are chosen so that both can be excited at the $532 \mathrm{~nm}$ laser wavelength while having minimal overlap of their fluorescence. The excitation probability of the dyes is not optimally matched but it was adequate to observe significant levels of aggregation. Improvement of the cross correlation sensitivity would be possible by adjusting the degree of labeling of the protein populations. These measurements were guided by the results from gel measurements of TLR4/MD2 oligomerization described below. Therefore we started with bulk liquid experiments with buffer conditions used in the gel experiments and observed oligomerization induced by LPS Ra mutant.

Initial bulk liquid experiments were performed starting with approximately $2 \mu \mathrm{M}$ total TLR4/MD2 concentration divided between the two dye-labeled protein samples. The protein was mixed with $10 \mu \mathrm{M}$ LPS and allowed to react for 30 minutes. The sample was then diluted to $<5 \mathrm{nM}$ concentration and FCS measurements were made immediately. Results are shown in Figure 4-21. The FCS measurements were made immediately after dilution because it was found in the gel experiments that the oligomers dissociated with time after dilution. 

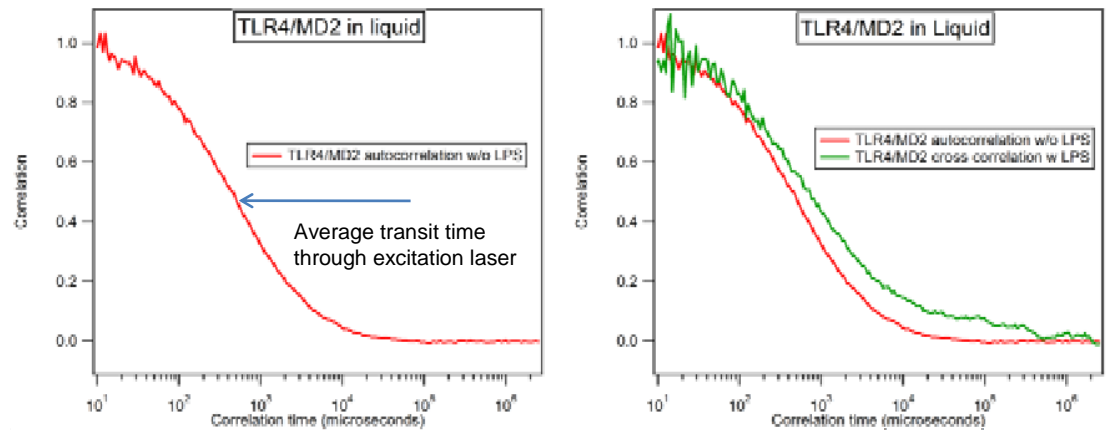

Figure 4-21. The plot on the left shows the normalized autocorrelation for TLR4/MD2 in solution without LPS added. On the right is a comparison between that autocorrelation and the normalized cross correlation for a sample taken after exposure to LPS. The cross correlation has substantial contribution from aggregates containing both dyes showing it is effective at detecting oligomerization in the sample.

After successfully implementing this approach in the liquid we began applying it to look for oligomerization of TLR4/MD2 bound to our model membrane. The membrane was loaded with a mixture of the two labeled TLR4/MD2 samples. We attempted to produce as high a concentration on the membrane as was compatible with FCS measurements, but further optimization of the concentration is possible. Results of the membrane measurements are shown in Figure 4-22. The shape of the cross correlation curve changes somewhat after LPS addition. The appearance of long correlation time components suggests the formation of oligomers. However, additional experiments are required to confirm this. Long correlation time features are sensitive to anomalous events such as individual large clusters being detected. In addition the uniformity of the membrane may be somewhat compromised at this high protein loading and this could also contribute long time features.
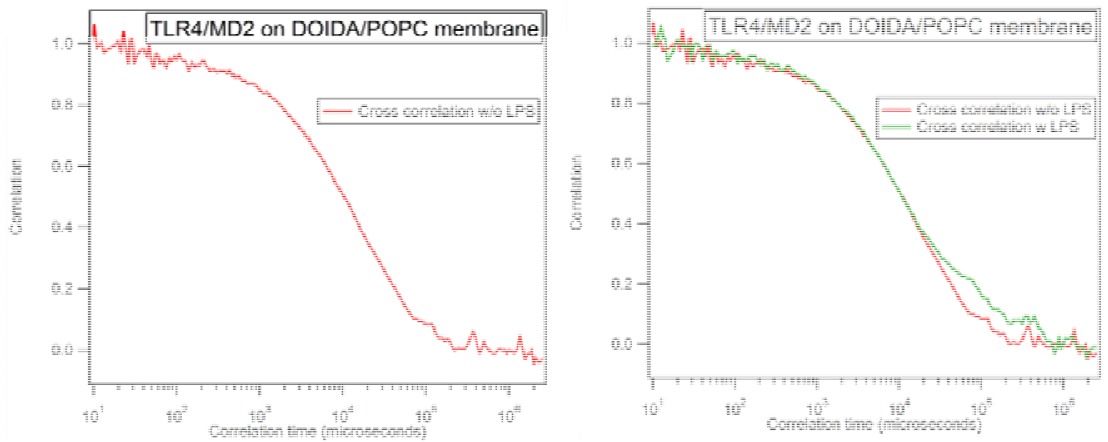

Figure 4-22. Normalized cross correlation measurements of a mixture of Alexa Fluor 514 and Atto 590 labeled TLR4/MD2 in the presence and absence of LPS. The cross correlation without LPS results from some crosstalk between the channels and has the same shape as the autocorrelation. The appearance of long time components in the cross correlation with LPS suggests oligomerization. 


\subsubsection{Results: TIRF}

Total Internal Reflection Fluorescence (TIRF) was also used to look for LPS-induced dimerization of TLR4/MD2 bound to lipid membranes. In this study two populations of His tagged TLR4/MD2 from R\&D were labeled with different colored fluorescent dyes (Alexa488 and Alexa532). The number of dye molecules per protein was $1+/-0.2$. The two samples were mixed together in equimolar amounts and then introduced to a TIRF cell with one glass slide coated with a bilayer of $10 \%$ DOIDA and $90 \%$ POPC. The total protein concentration was roughly $1 \mathrm{nM}$, but was adjusted for each sample to give optimal coverage (highest coverage that still allowed for tracking of individual membrane-bound proteins). Many sequences were collected in which the two colors were detected in separate channels simultaneously. Then LPS Ra mutant was introduced at room temperature and sequences were collected over a period of several hours. Sample images are shown in Fig 4-23. The sequences were analyzed for codiffusion of the two colors, either by visual inspection or using an analysis algorithm.
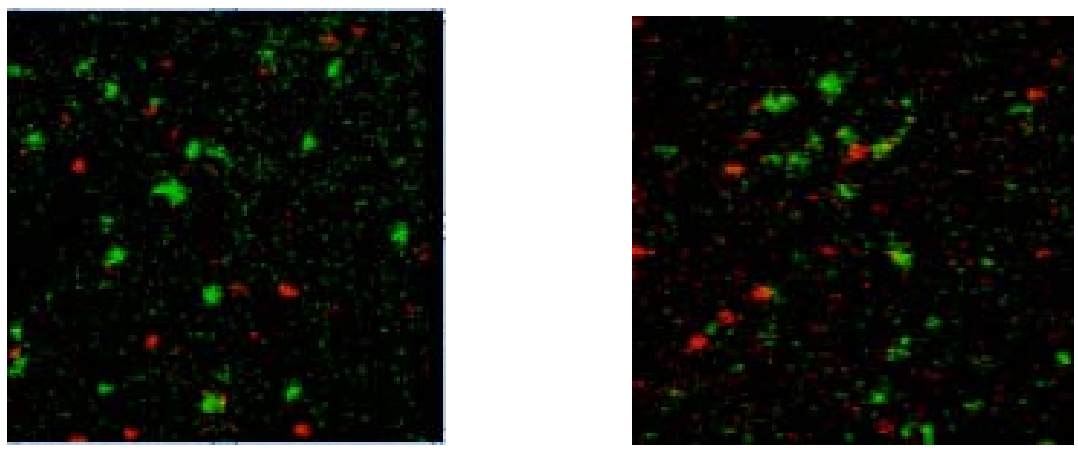

Figure 4-23. Sample still images of TLR4/MD2-488 and TLR4-MD2-532 bound to DOIDA/POPC membranes.

Early on this procedure was performed using $40 \mathrm{mM}$ PBS, $100 \mathrm{mM} \mathrm{NaCl}, \mathrm{pH}$ 7.4. and repeated using a range of LPS concentration and incubation times and also with CD14 and LBP present. Results of colocalization fraction showed low values and no detectable differences. Example:

no LPS mean $0.0085+/-0.0026$

with LPS mean $0.0052+/-0.0039$

Following the discovery that dimerization is greatly facilitated using $20 \mathrm{mM}$ Tris buffer, 200 $\mathrm{mM} \mathrm{NaCl}, \mathrm{pH}$ 8.0, a study was performed using the latter buffer with 5 uM LPS Ra mutant (no CD14 or LBP). Many sequences were collected with and without LPS, but no obvious increase in codiffusion or colocalization was detected.

For a control, we first added LPS Ra mutant to a solution of TLR4/MD2-488 and TLR4/MD2532, incubated for $3 \mathrm{hrs}$ at $37 \mathrm{C}$ to allow the complex to form, and then crosslinked with glutaraldehyde and added the solution to the TIRF cell. In that case the codiffusion of the two colors was obvious upon visual inspection. Sample images are shown in Fig 4-24. These sequences were used to test the efficacy of various analysis algorithms for codiffusion. 

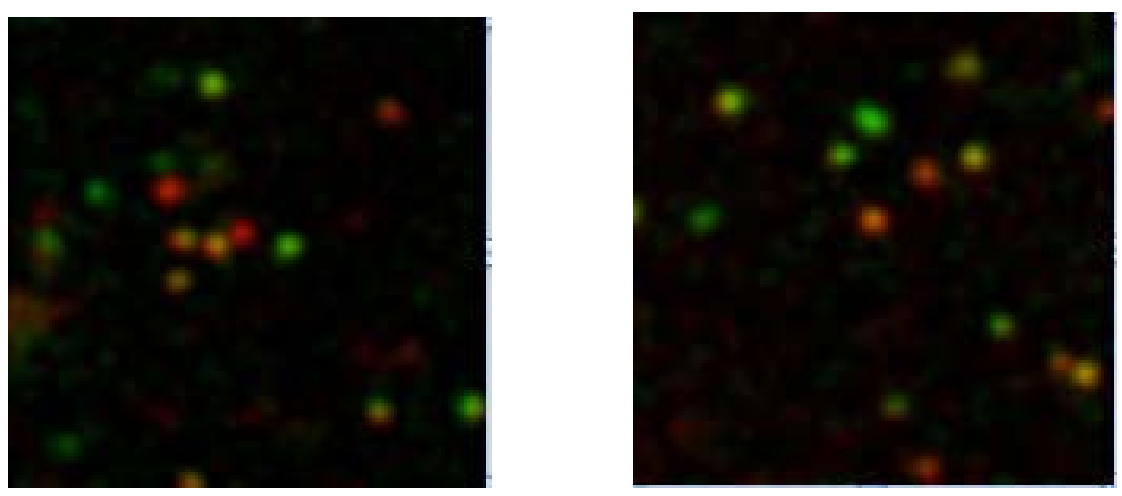

Figure 4-24. Sample still images of TLR4/MD2-488 and TLR4-MD2-532 bound to DOIDA/POPC membranes.

For all the conditions studied, introduction of LPS Ra mutant did not induce a detectable change in the amount of codiffusion. This is most likely due to the fact that receptor dimerization does not occur at the low surface densities that can be studied by this method. However, near the end of the program we discovered that addition of Cd14 and LBP together significantly facilitated oligomerization at much lower concentrations of soluble TLR4/MD2. Therefore, the TIRF study should be repeated under those conditions.

We performed a series of experiments in which the receptor was bound to the membrane at relatively high density where individual molecules could no longer be detected. After incubation with LPS Ra mutant, the amount of TLR4/MD2 bound to the membrane was gradually reduced by adding EDTA, which removes copper from the headgroups of DOIDA. At a sufficiently low density, single molecules could again be detected. Substantial crosslinked oligomers were detected. However, some crosslinked oligomers were also detected in the absence of incubation with LPS, indicating that the density of receptors was sufficiently high that at the glutaraldehyde concentration used, crosslinking occurred in absence of oligomerization. The method could possibly succeed with a lower concentration of glutaraldehyde or lower receptor density, but we were unable to do that within the time available for this program.

\subsubsection{Results: FRET}

In principle, it is possible to detect LPS-induced dimerization of TLR/MD2 bound to liposomes using FRET. For these experiments samples of TLR4/MD2 were labeled with Atto 532 and Atto $647 \mathrm{~N}$. These dyes were chosen to have a relatively large Förster radius, 59Å, but virtually no direct excitation of the acceptor. As a result a very small FRET efficiency was detected (control). Experiments were performed with a mixture of the two samples at a fairly high loading on the membrane, although not so high that mobility of the proteins ceased. In the absence of LPS no FRET was observed. Surprisingly, addition of LPS did not lead to a significant FRET signal. A similar result was seen in bulk samples at concentrations that lead to oligomerization in the gel experiments (discussed earlier in section 4.1.3). An explanation for this result is that perhaps the TLR4 complex is too large to be effectively detected in this fashion, but further experiments are warranted. 


\subsubsection{Results: CryoEM}

A final method used to try to detect LPS-induced dimerization of TLR/MD2 bound to membranes was CryoEM imaging. In this approach, His-tagged TLR/MD2 was bound to liposomes of DOIDA/Cu2+/POPC and the liposomes were captured on a TEM grid by rapid immersion of the grid in liquid nitrogen. EM images were obtained for the liposomes in absence of LPS Ra mutant and also after addition of LPS Ra mutant.

Since TLR4 is membrane bound protein, we decided to study it in its natural environment in cryoEM. That would allow us to image protein complexes in their "native" orientation relative to cell membranes. To mimic cell membrane we used $100 \mathrm{~nm}$ lipid vesicles (liposomes) made of mixture of DOIDA and POPC. POPC served as a filler lipid while DOIDA provided anchoring of his-tagged TLR4/MD2 complex to the liposomes. The liposomes were generated by D Sasaki. To confirm binding of TLR4/MD2 complex to DOIDA-containing liposomes the complex was labeled with gold using immuno-gold labeling and negative staining (2\% uranyl-acetate) in EM. TLR4/MD2 was incubated with MAB1787 antibody followed by labeling with gold-conjugated secondary goat anti-mouse antibody. Gold particles were $10 \mathrm{~nm}$ in size (Figure 4-25).

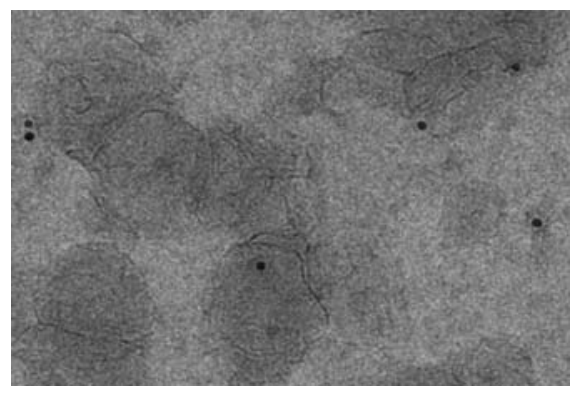

Figure 4-25. Image of negatively stained gold-labeled TLR4/MD2 bound to DOIDA-POPC liposomes. Black dots in the image correspond to gold clusters of $10 \mathrm{~nm}$ in size. In many cases the labels were in close proximity to the liposomes, which confirmed the binding of protein complex to the vesicles.

We then switched to cryoEM to select an optimal DOIDA concentration to provide anchoring for the proteins to liposomes. We found that with 10\% DOIDA/90\% POPC there were too many TLR4/MD2 complexes present in the liposomes making it difficult to box out individual complexes from the image for image processing while 1\% DOIDA/99\% POPC liposomes did not have any protein bound to them. With 5\% DOIDA/95\% POPC the proteins anchored to liposomes were distributed evenly around circumference of the vesicles and were sufficiently distant from each other to allow box out reasonable number of individual complexes bound to membrane for image processing and three-dimensional reconstruction (Figure 4-26).

One of the key questions to answer in the present study was TLR4/MD2 oligomerization with and without binding of LPS. We therefore decided to image TLR4/MD2 complex in the presence of LPS. Several experiments with LPS RA mutant (Sigma 19641) binding to TLR4/MD2 complex were performed, including binding of LPS dissolved in buffer straight from lyophilized state, binding of gold-labeled LPS to the complex and TEA to facilitate breakage of large 
oligomers of LPS and to prevent its aggregation in solution. In addition, PNGase F was used to remove sugars from TLR4/MD2 to improve the accessibility of LPS to the complex.
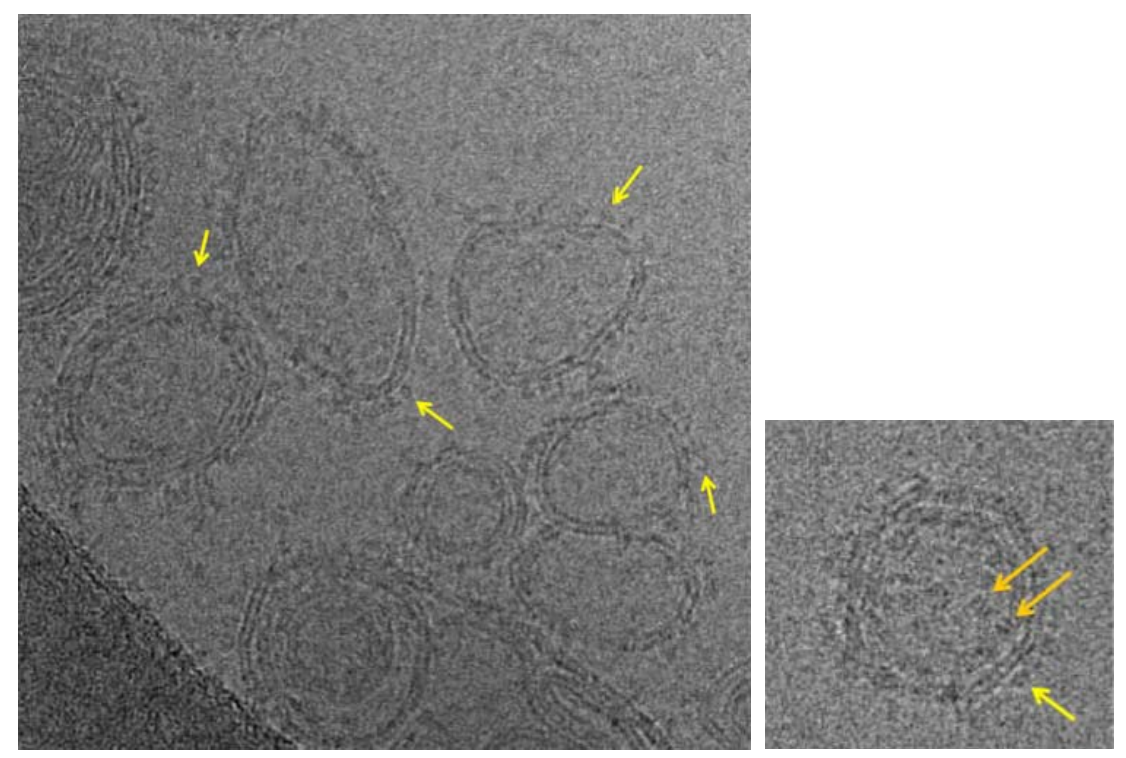

Figure 4-26. CryoEM images of his-tagged TLR4/MD2 bound to liposomes $(95 \% \mathrm{POPC}+5 \%$ DOIDA) yellow arrows point to side views of dimers of TLR4/MD2 complexes while gold arrows show top views of these dimers

To disperse LPS in buffer it was sonicated (using a probe sonicator) for $20 \mathrm{~min}$, followed by adding TEA ( $200 \mathrm{mM})$ to the solution and sonicating for 7 more min. LPS was then mixed with TLR4/MD2 $(1 \mathrm{mg} / \mathrm{ml})$ and the mixture was incubated at 37C for $3 \mathrm{hrs}$. After mixing the resulting solution with 5\% DOIDA/95\% POPC liposomes and incubating it at room temperature for 30 min $2 \mu \mathrm{l}$ of the solution was applied to carbon holey film grids (Protochips) and plunge-frozen in liquid ethane.

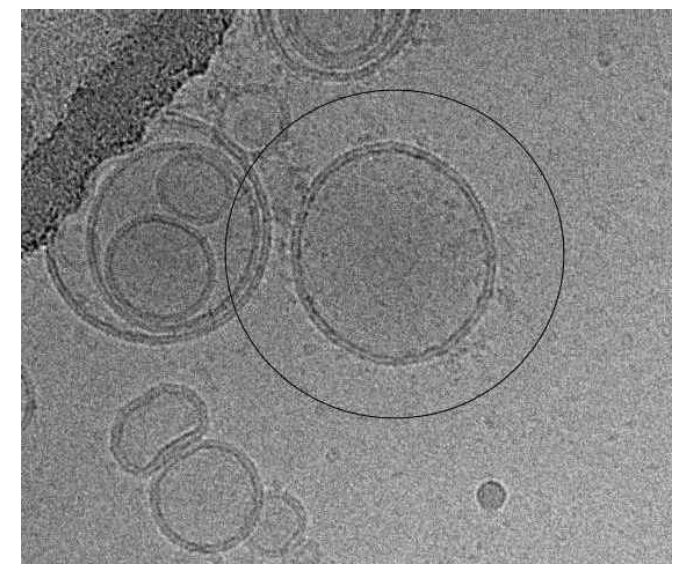

Figure 4-27. CryoEM images of TLR4/MD2 incubated with LPS followed by binding to liposomes. 
36,000 images of individual TLR4/MD2 complexes with part of the liposomes were selected from 113 CCD frames. The images were normalized to the same average density and standard deviation, aligned translationally and rotationally, classified and their relative angular orientations were determined using angular reconstitution technique from IMAGIC program package (van Heel, M., G. Harauz, et al. (1996)) similar to the image processing steps performed earlier (see above). The result is shown in Figure 8. Unexpectedly we found that TLR4/MD2 formed dimers when binding to liposomes, although the dimers formed differently from previous structures determined by X-ray crystallography (see Figure 8 caption). We plan to refine the reconstruction and publish results in a peer-refereed journal. In addition LPS binding to TLR4/MD2 complexes will be studied in more detail including 3D reconstruction.

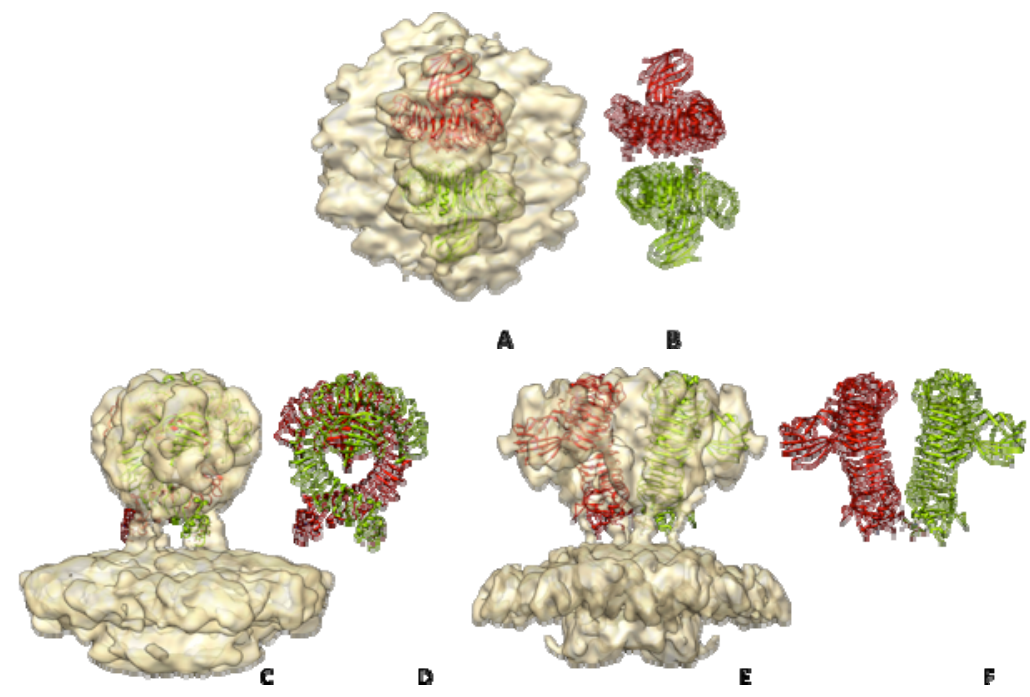

Figure 4-28. Surface rendering of cryoEM reconstruction of dimers of TLR4/MD2 complexes bound to lipid membrane. Top view is shown in A with X-ray structure (Kim, H.M., et al., Cell, 2007. 130, p. 906-17) of TLR4/MD2 fitted into the reconstruction. Dimer of TLR/4MD2 is shown in the same orientation in B without CryoEM map. C and E show side views of cryoEM map with X-ray structure of TLR4/MD2 fitted. D and F show just dimers fitted into cryoEM map. Note that dimers are different from published in Park, B.S., et al., Nature, 2009. 458, p. 1191-5 


\section{SUMMARY}

We developed methods to determine the association constant for oligomerization of the soluble receptor. LPS-induced oligomerization was observed to be a strong function of buffer conditions. In $20 \mathrm{mM}$ Tris $\mathrm{pH} 8.0$ with $200 \mathrm{mM} \mathrm{NaCl}$, the onset of receptor oligomerization occurred at $0.2 \mathrm{uM}$ TLR4/MD2 with E coli LPS Ra mutant in large excess. In the presence of $0.5 \mathrm{uM}$ CD14, the onset of receptor oliomerization was unchanged. However, in the presence of $0.5 \mathrm{uM}$ LBP the onset of receptor oliomerization occurred at much lower concentration of TLR4/MD2. In the presence of $0.5 \mathrm{uM} \mathrm{CD14}$ and $0.5 \mathrm{uM} \mathrm{LBP}$, the onset of receptor oligomerization was observed to be less than $10 \mathrm{nM}$ TLR4/MD2. These are the major findings of this work. The fact that LBP alone, but not CD14 alone, resulted in a decrease in the TLR4MD2 concentration for the onset of LPS-induced oligomerization is very important. The effect of LBP may be due to LBP extracting LPS from micelles and shuttling it to TLR4/MD2. However, since CD14 alone has no apparent affect on the association of the receptor, it is not clear what role CD14 plays. Yet CD14 has been shown to have a critical effect on TLR4 signaling (Godowski, Nature Immunol. 2005; Jiang, Nat. Immunol. 2005). More work is needed to understand this.

In the course of the abovementioned work we discovered that substantial amounts of higher order oligomers are present in addition to dimers. A new method was developed to quantify the amount of oligomer relative to TLR4/MD2 monomer, allowing determination of equilibrium association constants.

Several methods were pursued to study LPS-induced oligomerization of the membrane-bound receptor, including CryoEM, FRET, colocalization and codiffusion observed by TIRF, and FCS. However, these approaches met with only limited success during the course of this program. The primary difficulty with the fluorescence imaging methods (TIRF and FCS) that involve the membrane-bound receptor is that these methods only work at relative low coverages such that single molecules can be detected. Yet we did not observe LPS-induced oligomerization of the receptors at such low coverages, consistent with the fact that 0.2 uM TLR4/MD2 was required for oligomerization of the soluble receptor. However, at the end of the program we discovered that with LBP alone or with both CD14 and LBP present, LPS-induced oligomerization occurs at much lower concentrations $(<10 \mathrm{nM})$ of the soluble receptor. It stands to reason that oligomerization of the membrane-bound receptor should occur at substantially lower coverages with CD14 and LBP present also. Therefore, we expect that these fluorescence imaging methods would likely be successful if performed with CD14 and LBP present. 
6. REFERENCES 


\section{DISTRIBUTION}

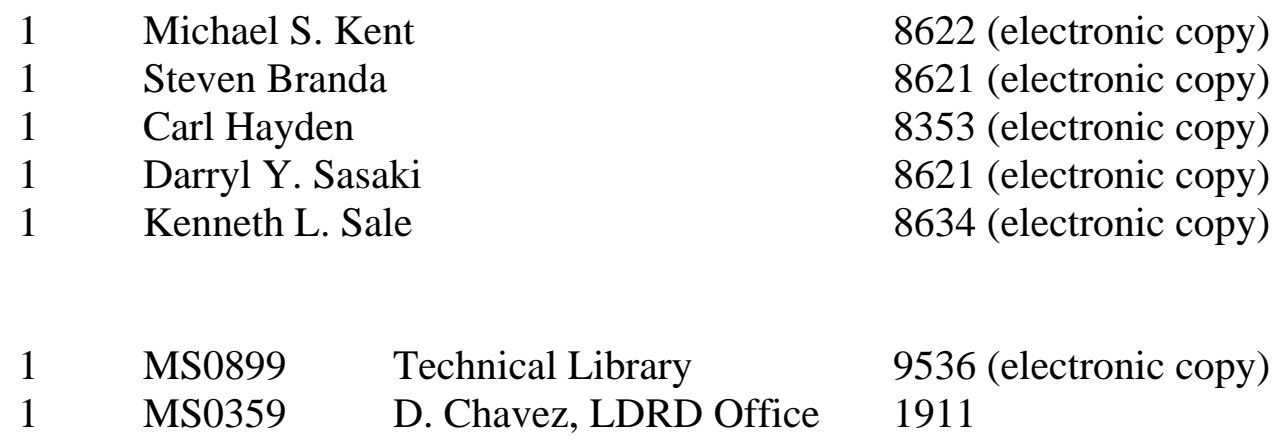




\section{Sandia National Laboratories}

